\title{
WestVirginiaUniversity
}

THE RESEARCH REPOSITORY @ WVU

Graduate Theses, Dissertations, and Problem Reports

2003

\section{Plant-wide energy assessment using a systems approach}

\author{
Rajkumar Selvaraj \\ West Virginia University
}

Follow this and additional works at: https://researchrepository.wvu.edu/etd

\section{Recommended Citation}

Selvaraj, Rajkumar, "Plant-wide energy assessment using a systems approach" (2003). Graduate Theses, Dissertations, and Problem Reports. 1415.

https://researchrepository.wvu.edu/etd/1415

This Thesis is protected by copyright and/or related rights. It has been brought to you by the The Research Repository @ WVU with permission from the rights-holder(s). You are free to use this Thesis in any way that is permitted by the copyright and related rights legislation that applies to your use. For other uses you must obtain permission from the rights-holder(s) directly, unless additional rights are indicated by a Creative Commons license in the record and/ or on the work itself. This Thesis has been accepted for inclusion in WVU Graduate Theses, Dissertations, and Problem Reports collection by an authorized administrator of The Research Repository @ WVU. For more information, please contact researchrepository@mail.wvu.edu. 


\title{
Plant-Wide Energy Assessment using a Systems Approach
}

\author{
Rajkumar Selvaraj \\ Thesis submitted to the \\ College of Engineering and Mineral Resources \\ at West Virginia University \\ in partial fulfillment of the requirements \\ for the degree of \\ Master of Science \\ in \\ Industrial Engineering
}

B. Gopalakrishnan, Ph.D., Chair Ralph Plummer, Ph.D. Richard Turton, Ph.D. Ken Baxter

Department of Industrial and Management Systems Engineering

$$
\begin{gathered}
\text { Morgantown, West Virginia } \\
2003
\end{gathered}
$$

Keywords: Audit, Performance Evaluation, Methods Study, Energy, Systems Analysis 


\section{ABSTRACT \\ Plant-Wide Energy Assessment using a Systems Approach}

\section{Rajkumar Selvaraj}

Energy plays a central role in the operation of the industrialized U.S. economy. Industry is historically the largest consuming sector of the economy with more than 60 percent of the energy consumed for manufacturing. The U.S. chemical industry is the world's largest producer of chemicals producing over 25 percent of the total production worldwide. In a chemical industry, the energy is used to supply heat and power for manufacturing processes and for energy feedstock. Due to the energy intensive nature of chemical industries, energy assessments performed could yield huge savings in energy and cost.

The aim of this research is to develop a standardized methodology for performing plant-wide energy assessments using a systems approach. If there is no standard methodology, the assessment team will easily get overwhelmed with the amount of data in the plant and might be focusing on insignificant areas for energy saving opportunities. This methodology would help the plant personnel to conduct in-house energy assessments. 


\section{ACKNOWLEDGEMENTS}

I would like to wholeheartedly thank my advisor Dr. B. Gopalakrishnan for his continued support, guidance and encouragement during the course of this research work. I also wish to thank Dr. R. Turton, Dr. Ralph Plummer and Mr. Ken Baxter, my committee members, for their advice and support.

Above all, I wish to thank God, my parents and all my friends for their constant support and blessings and enabling my success and happiness in all my pursuits and endeavors in life. 


\section{Table of Contents}

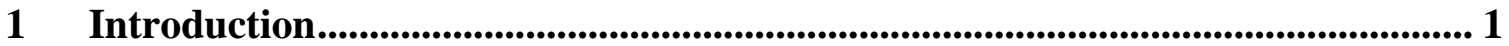

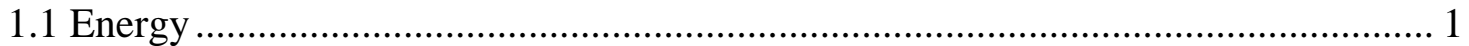

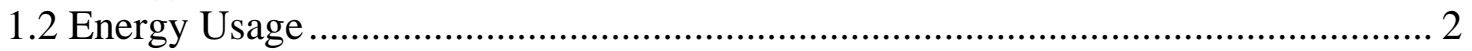

1.3 Industrial Energy Conservation …………………............................................... 5

1.4 Corporate Viewpoint........................................................................................ 7

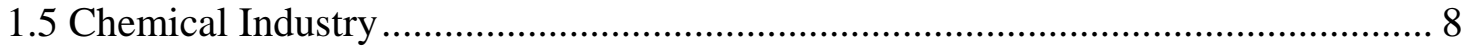

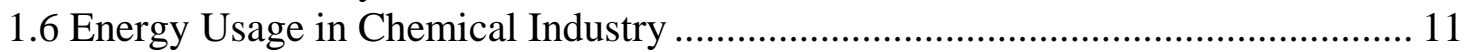

1.7 Demographics of Energy Usage ............................................................................. 12

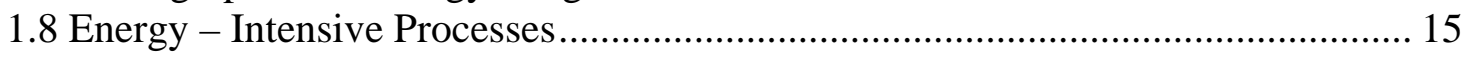

1.9 Need for Energy Assessments in Chemical Industry.............................................. 16

1.10 Industrial Assessment Center (IAC) ………….................................................. 17

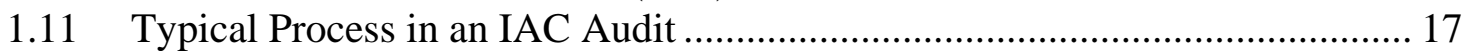

1.12 Why Typical Process may not be enough....................................................... 18

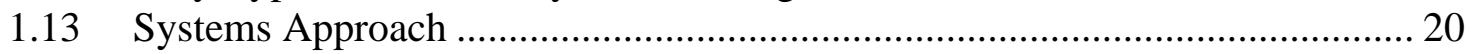

1.14 Plant-wide Energy Assessments ..................................................................... 22

1.15 Systems Approach to Plant-wide Energy Assessments ...................................... 23

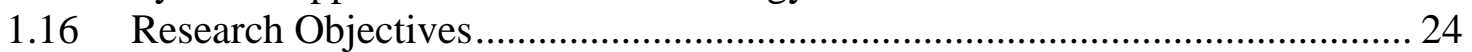

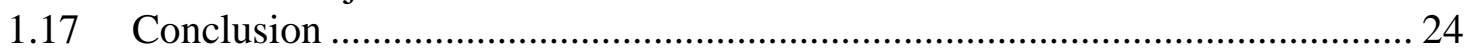

2 Literature Review ....................................................................................................... 25

2.1 Energy Conservation........................................................................................ 25

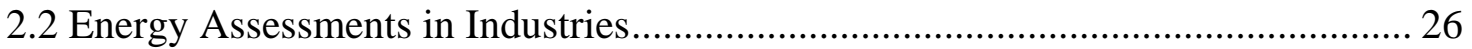

2.3 Energy Assessment in Chemical Industry .......................................................... 29

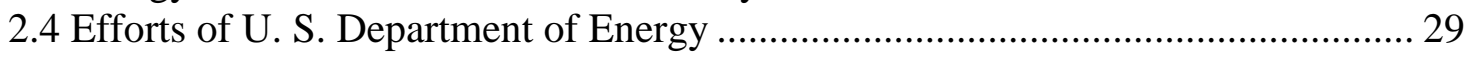

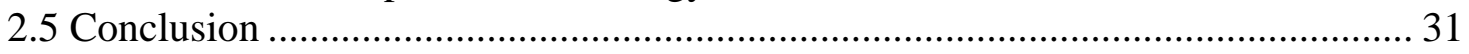

3 Plant-wide Energy Assessment Methodology........................................................... 32

3.1 Difference between a One-day Assessment and a Plant-wide Energy Assessment 37

3.2 Knowledge and Expertise Acquisition................................................................. 38

3.3 Pre-assessment Plant Data Collection.................................................................... 40

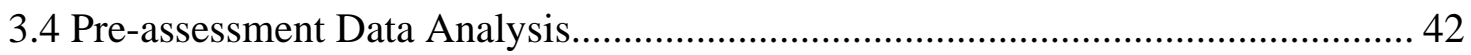

3.5 Corporate Knowledge Acquisition ..................................................................... 46

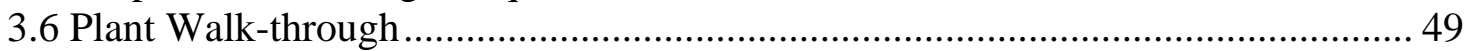

3.7 Data Analysis Modification ................................................................................... 49

3.8 Energy Assessment Plan ....................................................................................... 49

3.9 Onsite Data Gathering.......................................................................................... 55

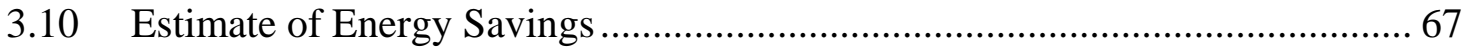

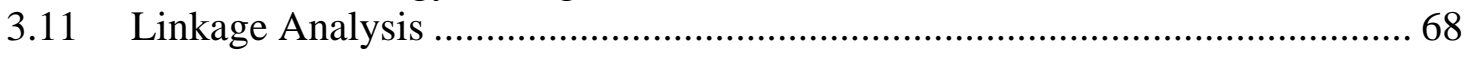

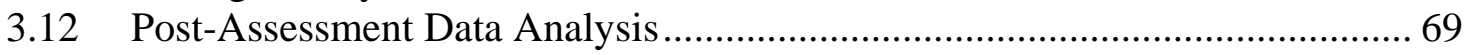

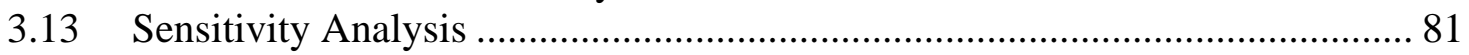

3.14 Final Report and Corporate Analysis................................................................ 87

3.15 Implementation and Continuous Improvement ................................................ 88

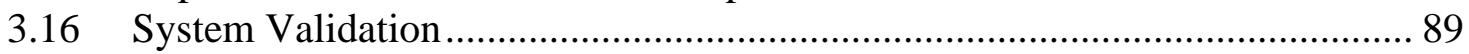

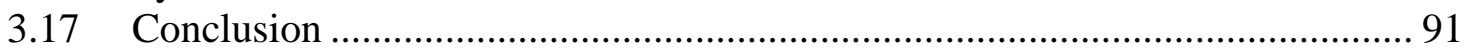

4 Conclusion and Future Work .............................................................................. 92

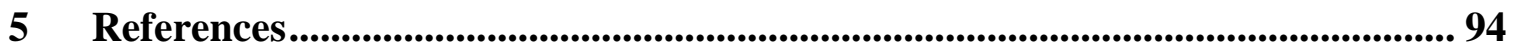




\section{List of Tables}

Table 1.1: Economic and Trade Statistics of the Chemical Industry in 1997 [1] ............ 10

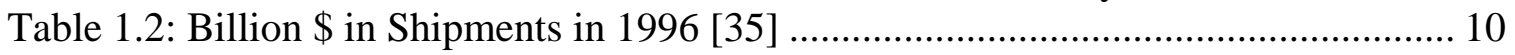

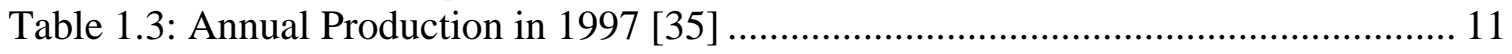

Table 3.1: Project Milestone Chart for the Energy Assessment ...................................... 51

Table 3.2: Data collection template for pipe and surface insulation................................ 52

Table 3.3: Template for assessing steam leaks ......................................................... 53

Table 3.4: Template for assessing the lighting in the plant ......................................... 53

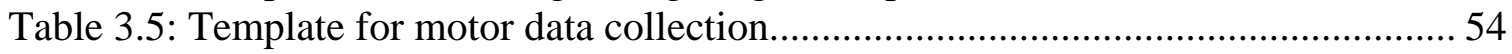

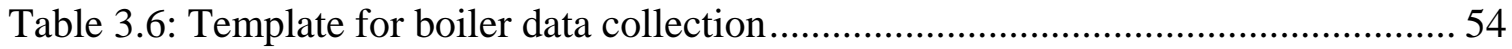

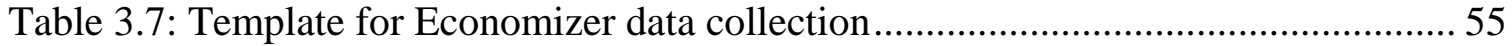

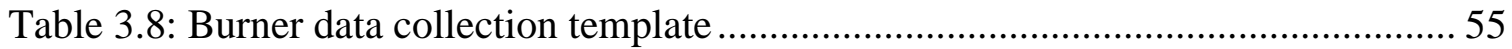

Table 3.9: Sample of the data collected on Insulation ................................................ 57

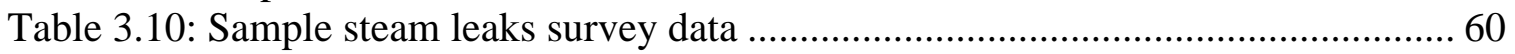

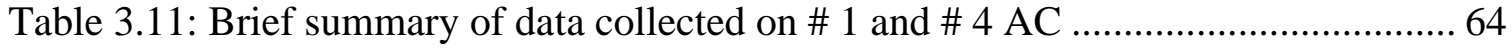

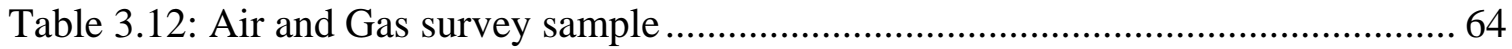

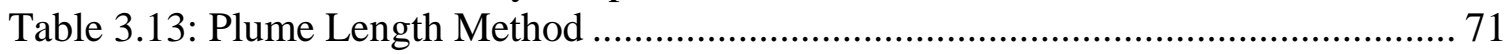

Table 3.14: Existing arrangement of the compressors............................................... 74

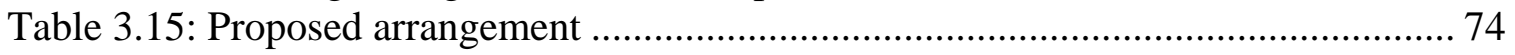

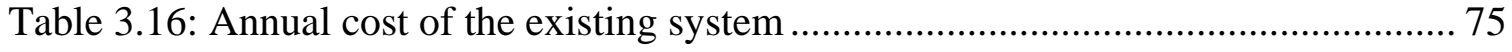

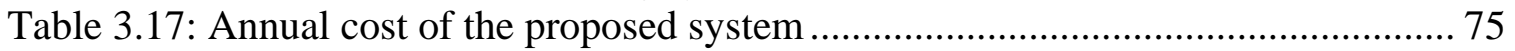

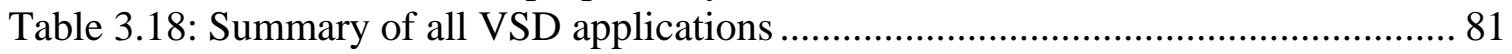

Table 3.19: Boiler Efficiency Sensitivity Analysis.................................................... 82

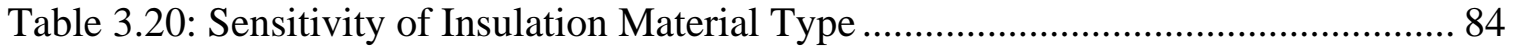

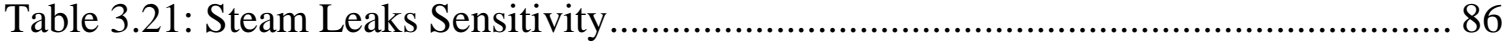




\section{List of Figures}

Figure 1.1 Energy Production by Source for 2000 in U.S. [5] ........................................ 2

Figure 1.2: Overview of Energy Production and Consumption [5] .................................. 3

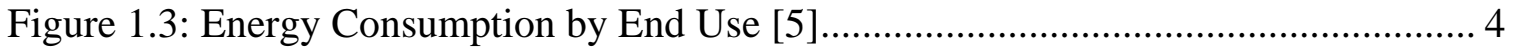

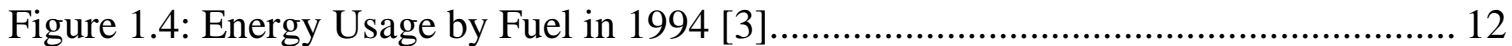

Figure 1.5: Heat and Power Consumption of Energy in 1994 [35] ................................. 13

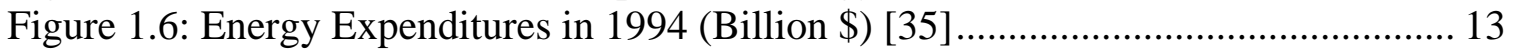

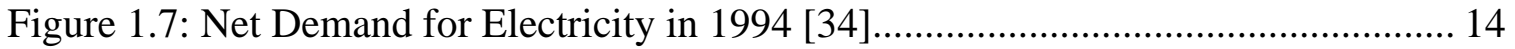

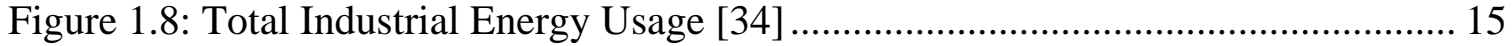

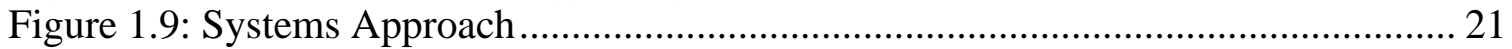

Figure 3.1: Methodology for Plant-wide Energy Assessments ...................................... 32

Figure 3.2: Methodology for Plant-wide Energy Assessments (continued) ...................... 33

Figure 3.3: Screening of Energy Assessment Plan ....................................................... 50

Figure 3.4: Thermal image of uninsulated pipe and flange ......................................... 58

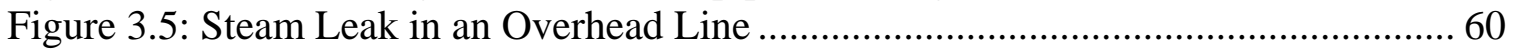

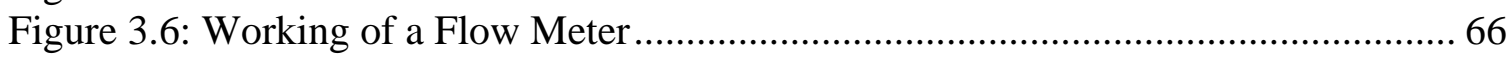

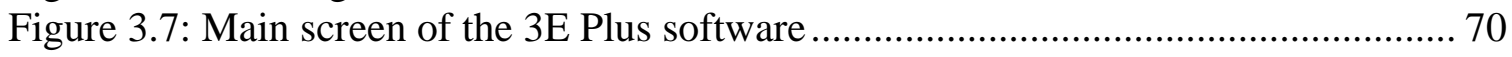

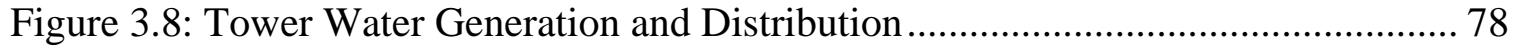

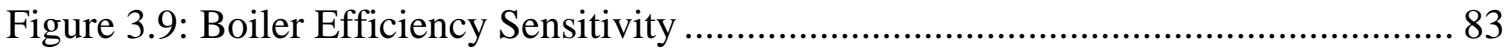

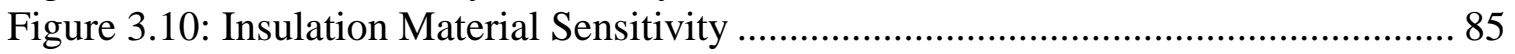

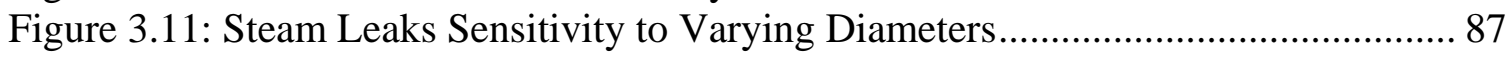

Figure 3.12: Methodology for Smaller Facilities (excluding the shaded areas) ............... 90 


\section{Introduction}

\subsection{Energy}

One of the most fundamental parts of our universe is energy. The last half of the twentieth century will be remembered by historians for energy. For centuries the abundantly and readily available, low-cost energy was used for all the requirements without any thought for conservation. Between 1974 and 1994, the amount and share of fuel oil use have declined substantially. In the mid-1970's, oil prices rose substantially as a result of limited supplies of crude oil used to make refined products [3]. Warnings that had been given for a long time took a major aspect, as fuel shortages and rising costs nearly paralyzed the industrial economies and literally shocked the world into an inflationary period that has not yet ended [1].

In reality the problem was much more complex involving not only oil prices but also the uneven geographical distribution of energy resources, the exponential growth of population and fuel consumption, political and national security considerations and long term environmental effects [1]. When energy problems caused by the rapid increase in demand in the face of dwindling fuel supply first became apparent, the immediate response was to seek new supplies and alternative fuels [2].

Later, supply problems were eased by giving consideration to the user as a means of conserving fuels and capital by improving end-use efficiency. Approaching the energy problems from the user's side rather than the supply side introduces new problems. The problem is complicated by the fact that there are more number of users than the suppliers. Due to their number and diversity, it is difficult to communicate with the users. End users are not readily approached by regulatory or legislative controls due to their diversity. Technical improvements are limited because the technological sophistication and the capital resources vary widely with the end users. A large number of different technologies, materials and equipment are necessary due to the infinite variety of uses.

Changes made by users are advantageous in that the impact on energy use is immediate or short term, compared to the long term payback needed to add energy supply capacity. The economic signals of rising fuel prices give a positive encouragement to the users to reduce or eliminate all unnecessary uses of energy. Even though there is 
considerable diversity in the end use technology, certain basic approaches or principles apply to a wide variety of applications.

\subsection{Energy Usage}

A majority of the energy produced in the United States comes from fossil fuels, namely coal, natural gas, and crude oil, as in the rest of the industrialized world (Figure 1.1). In the year 2000, the energy produced from fossil fuels accounted for 80 percent of the total energy production and were valued at an estimated $\$ 148$ billion nominal dollars [5].

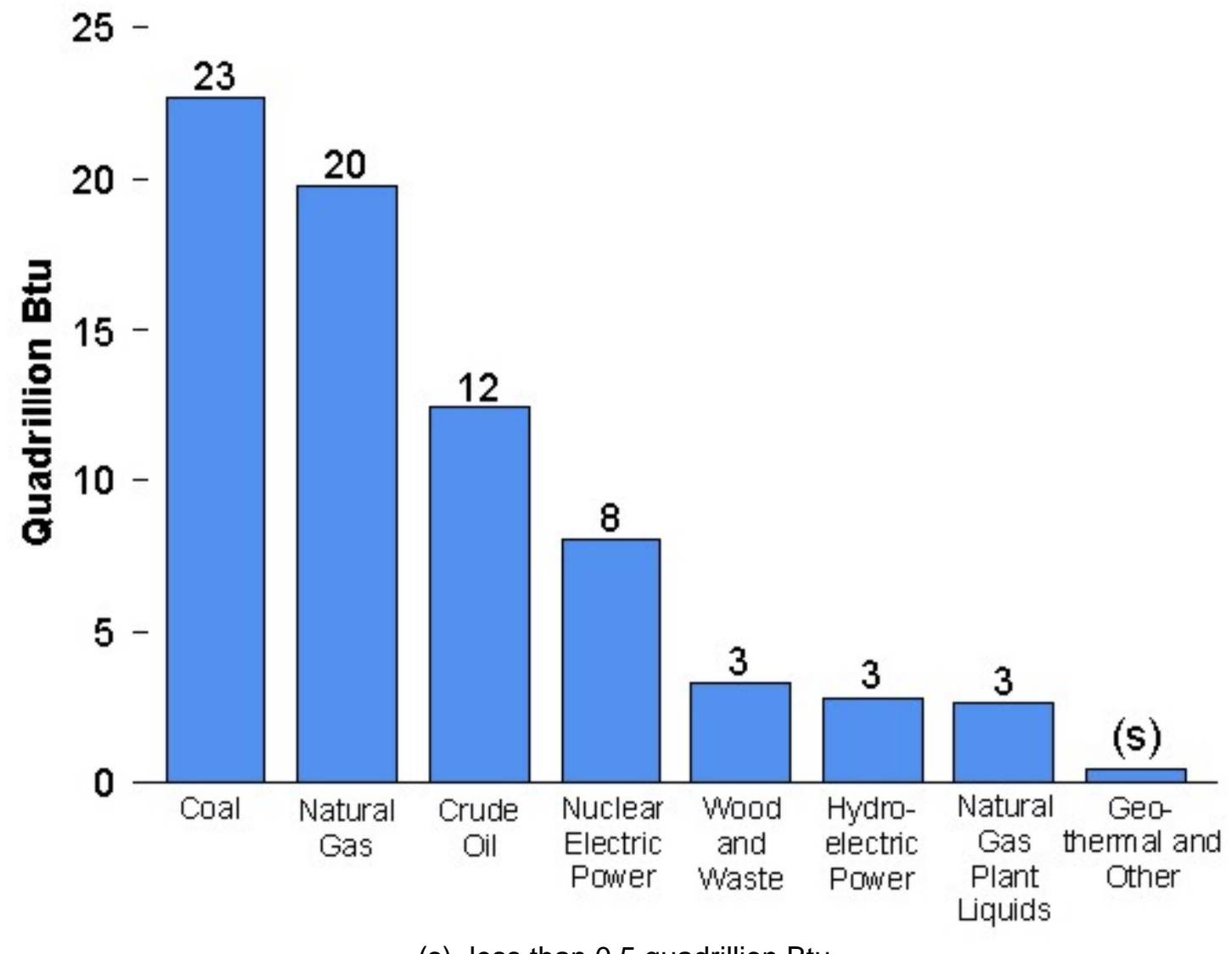

(s)=less than 0.5 quadrillion Btu

Figure 1.1 Energy Production by Source for 2000 in U.S. [5]

Although small amounts of coal were imported from Britain in colonial times, the United States was mostly self-sufficient in energy for much of its history. Production and 
consumption of energy were nearly in balance up to the late 1950s. Over the following decade, the consumption slightly outpaced the domestic production and by the early 1970s a more significant gap had developed (Figure 1.2). In 2000, the United States produced just under 72 quadrillion British thermal units (Btu) of energy and exported roughly 4 quadrillion Btu. Consumption totaled about 98 quadrillion Btu, requiring imports of almost 29 quadrillion Btu. The efficiency with which Americans use energy has improved over the years. Efficiency improved 49 percent between 1949 and 2000, as the amount of energy required to generate a dollar worth of output (chained 1996 dollars) fell from 20.6 thousand Btu to 10.6 thousand Btu. Nevertheless, a growing population and economy increased energy usage [5].

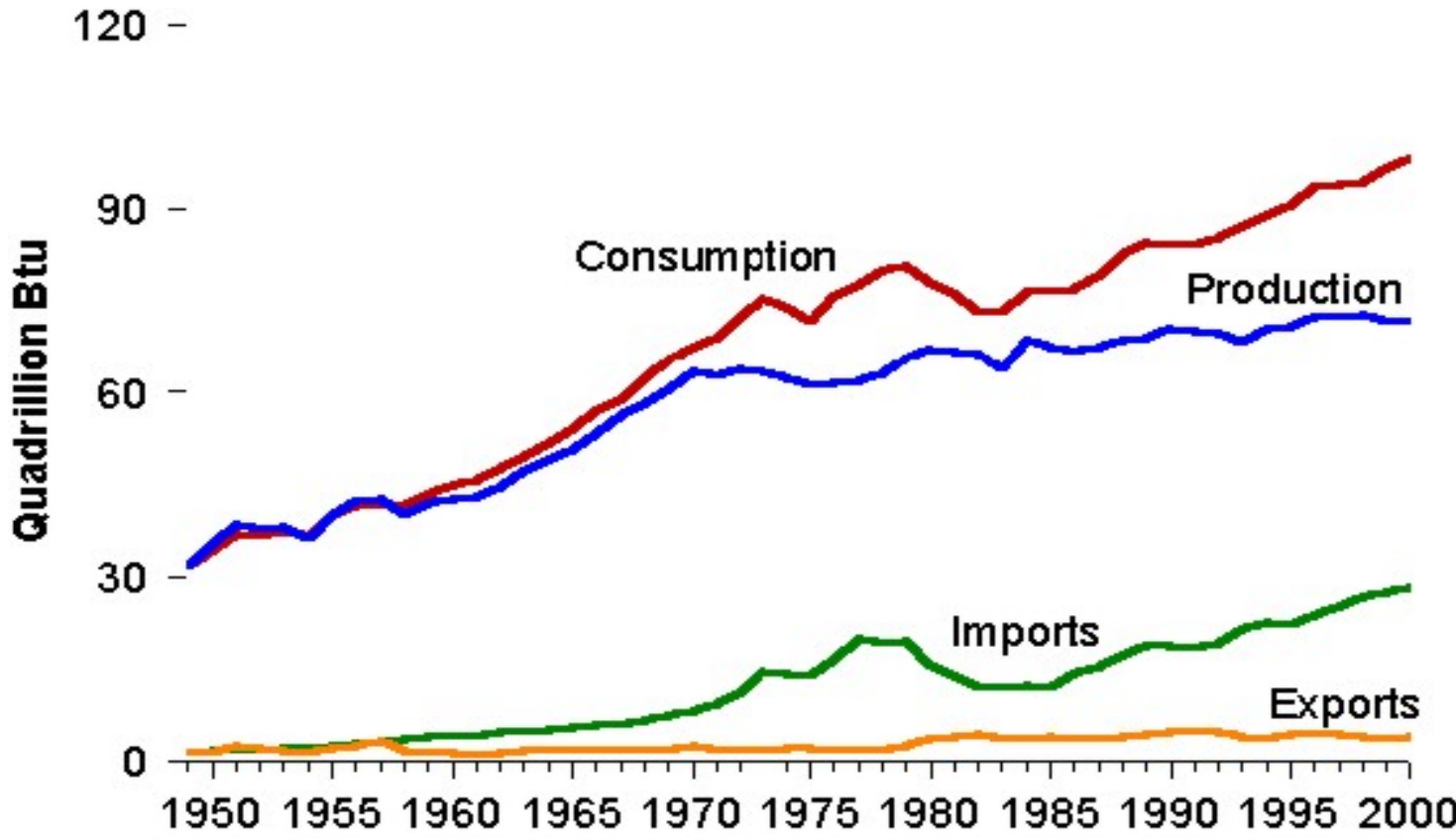

Figure 1.2: Overview of Energy Production and Consumption [5]

Energy plays a central role in the operation of the industrialized U. S. economy. In the recent years, the American consumers have spent over half a trillion dollars a year on energy which is consumed in four broad sectors, namely residential, commercial, industrial, and transportation. Industry is historically the largest consuming sector of the economy with fluctuating trends, compared to the smooth trends in the other sectors (Figure 1.3) [5]. 


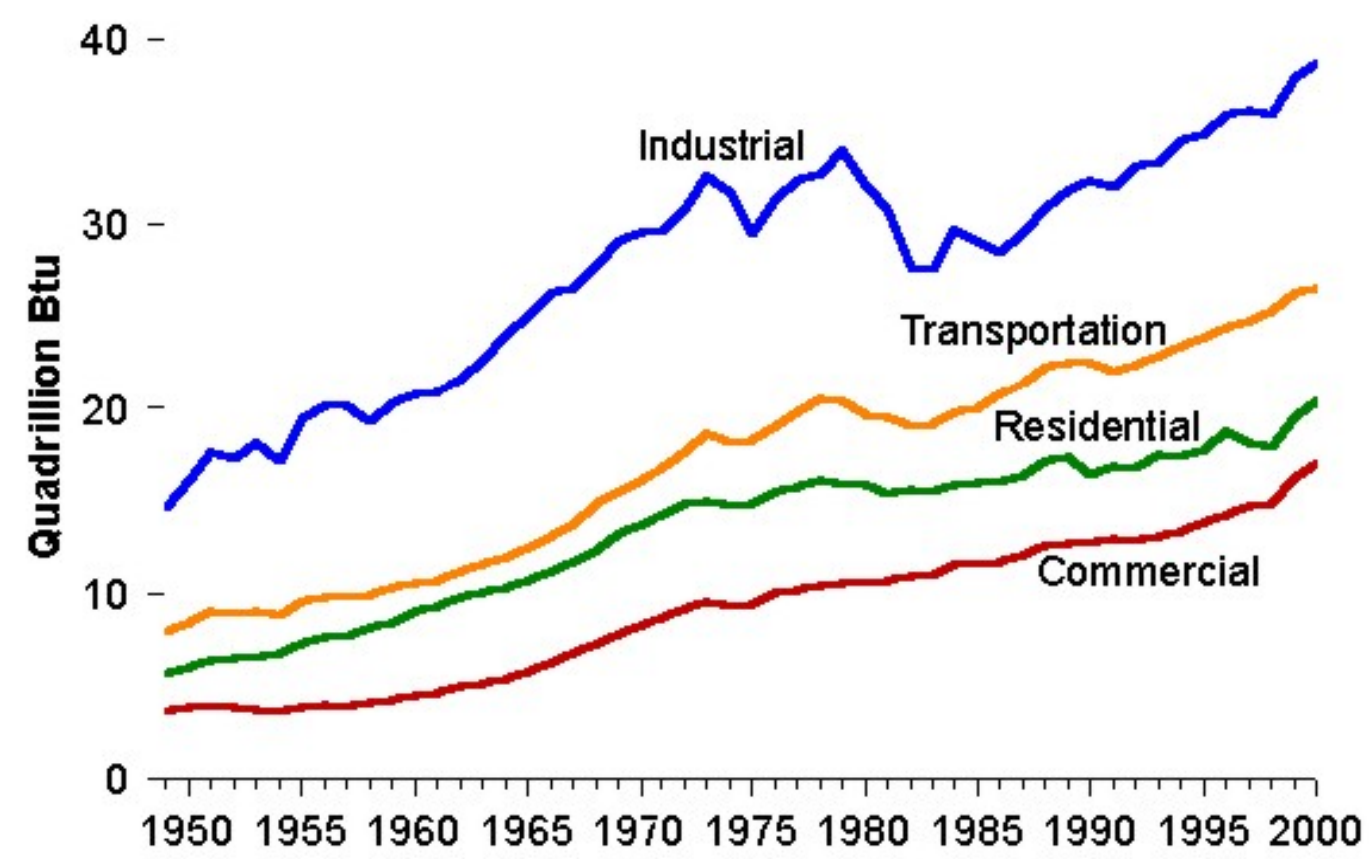

Figure 1.3: Energy Consumption by End Use [5]

About 60 percent of the energy consumed in the industrial sector is used for manufacturing. The remainder goes to mining, construction, agriculture, fisheries, and forestry. Within manufacturing, large consumers of energy are the petroleum and coal products, chemicals and allied products, paper and allied products, and primary metal industries. Natural gas is the most commonly consumed energy source in manufacturing. The predominant end-use activity is process heating, followed by machine drives and then facility heating, ventilation, and air conditioning combined [5].

Among the most significant environmental effects of energy production and consumption is the emission of greenhouse gases. These gases, carbon dioxide, methane, nitrous oxide, and others, block infrared radiation from the Earth to space and retain the captured heat in the atmosphere. This greenhouse effect keeps the Earth's climate hospitable to life. In recent years human-caused additions to greenhouse gases are raising global average temperatures and may produce harmful changes in the global climate. Energy-related emissions form a significant portion of these additions. Carbon dioxide $\left(\mathrm{CO}_{2}\right)$ accounts for the largest share of combined human-caused greenhouse gas 
emissions. In 1999, it totaled about 5.6 billion metric tons of $\mathrm{CO}_{2}$ gas, 17 percent higher than in 1980 [38]. All sectors of the U.S. economy contribute to energy-related greenhouse gas emissions, especially $\mathrm{CO}_{2}$. In 1999 energy-related $\mathrm{CO}_{2}$ emissions, the industrial and transportation sectors each accounted for about one-third, the residential sector for about one-fifth, and the commercial sector for the remainder [38].

\subsection{Industrial Energy Conservation}

Energy is an integral component of modern industrialized society. One of the key aspects in energy conservation is to use energy more efficiently, particularly in those areas where the greatest use occurs. Better insulation of hot surfaces is one way in which major energy savings could be obtained [4]. Important areas that need to be given consideration in the context of industrial energy usage are:

- Industrial energy usage patterns and future outlook

- How the energy is used by industries

- Technologies to improve energy efficiency

- Corporate viewpoint on energy and the various incentives offered

One of the largest consumers of the energy produced is the industry. U.S. manufacturing plants, mines, farms and constructions firms currently consume about 25 quads (quadrillion British thermal units or Btu) of energy each year [4], which is about 42 percent of the nation's total consumption of energy. Industry thus has a major role in making the U.S. more energy efficient. Industrial energy use and the opportunities for improving its energy efficiency depend on many technical, economic, institutional and political factors. Industries such as petroleum refining, chemicals, primary metals, pulp and paper, food, and ceramics and glass, which physically or chemically convert matter, are the largest users of energy. They account for about 68 percent of the total industrial energy use [4].

Energy could be saved by industries by adopting many energy efficient technologies and practices, both currently available and under development. A few of the cost effective methods to improve energy efficiency are the following:

- General housekeeping and maintenance programs

- Energy management and accounting programs 
- Improved method and procedures for existing production methods

- New and better production methods

- Product changes

Though most of the equipment and process enhancements are specific to particular industries, there are several technologies like heat recovery technologies, high efficiency motors, variable speed drives, cogeneration, etc., that have applications in many industries. The cost and benefits of various energy efficient improvements vary widely. Minor operational changes, such as house keeping and maintenance, are typically the cheapest, easiest to implement, least risky and usually, though not always, yield the smallest energy and cost savings. Production equipment changes and energy conservation add-on technologies involve larger investments, typically $\$ 100,000$ to tens of millions of dollars, and may or may not be justified by reduced energy costs alone. Major process changes often require building a new facility, at costs typically exceeding \$ 100 million, and usually are justified only by strategic, market development concerns [2]. Energy savings are seldom sufficient to justify the investments of this magnitude.

The greatest increase in efficiency will come not from the direct efforts to reduce energy consumption, but by pursuing other economic goals like improved product quality, lower capital and operating costs, or specialized product markets. In many projects energy efficiency gains are obtained as a secondary consequence. For example, steel makers have installed continuous casters more for the improved product yield than the energy savings. Metal stamping plants have implemented new techniques for cushioning presses not for the $10 \%$ energy savings, but for the consistent products and lower maintenance cost [1]. Sometimes, however, pursuing improved quality or specialized markets can diminish energy efficiency. Such is the case in petroleum refining where several factors have combined to increase the energy requirements per unit of product in recent years.

There are various potential areas that can be identified for energy conservation measures, namely lighting, boilers, motors and pumps, destratification, insulation of steam lines and process equipment, air and steam leaks, and compressors. If we consider lighting in facilities, simply turning off unnecessary lights, day and night, and making greater use of available daylight for illumination saves energy for both lighting and air- 
conditioning with no added costs. Any percentage increase in seasonal boiler efficiency directly reduces fuel consumption in the same proportion. The steam and air leaks in the distribution lines contribute to direct loss of energy from the system. Steam leaks will cause an increase in the boiler load and makeup water consumption, as the steam lost has to be compensated by an addition of steam in the system to meet the needs. The loss associated with a steam leak is identical to that of vented steam [6]. If we consider the three phase-induction electrical motors in the facilities, usually the industrial personnel lack prior knowledge about the high efficient motors available in the market and the cost savings that can result from a replacement schedule. Another example would be the employment of de-stratification fans in the workplaces where heating units have been set up in place but comfort level has not been attained at work level. Several cases can be presented about better utilization of energy, but the purpose of this work would be to create awareness about the benefits of energy conservation.

\subsection{Corporate Viewpoint}

An important factor for industrial energy usage and efficiency is the corporation's internal cultures and external relationships. Technical and economic feasibility are the most commonly studied factors influencing energy efficiency investments while the company's general willingness to invest in process improvements, their energy awareness, and their access to information also have important impacts. One of the most important factors influencing energy efficiency is the willingness of the firms to invest in new technologies. Capital investment in modern equipment usually enhances energy efficiency, even when efficiency is not the primary purpose of the investment.

Even if the company is willing to invest, another hurdle for the managers is to know how the energy is being used in their plant and the various technologies available to improve the situation. Industries have direct financial incentives for reducing their energy costs by improving their energy efficiency. In industries such as steel, aluminum, cement and industrial gases, where energy is a major portion of the total costs, concerns about energy efficiency are high.

Cutting energy costs via technical means is not a high profile concern in most industrial companies. An operations manager's top priorities are keeping the production 
line up and running smoothly, making products that meet the consumer's specifications and expectations, and meeting regulatory guidelines. Energy costs tend to be secondary concerns. The general lack of concern about energy costs in many corporations is a major barrier to implementation of energy efficiency improvements.

There are many situations where there are new technologies which give production benefits in addition to energy savings. They are implemented primarily to boost product quality, further automate production or enhance some other characteristics and improve energy efficiency as a side benefit.

Information regarding new technologies and their energy characteristics should be available conveniently. Managers, especially those in small firms, do not have the time and the resources for gathering and analyzing large amounts of information to support their decisions. This is particularly true when equipment fails and needs immediate replacement. There is little time to research the available best replacement technologies, and then test and tune them up once they arrive. Consequently, in these situations the managers usually stick to the technologies that they know well and have used before. The State and the Federal governments are involved in providing information. Utilities are also involved in dissemination information as well as conducting assessments to inform companies about energy saving opportunities. Technological and economic feasibility are driving factors as well. Technologies must not only work successfully, but also be reliable, serviceable and proven. In addition they must be economical with respect to capital outlays, energy and other input prices and costs of capital.

Energy and energy management have been in the limelight in various manufacturing and service operations across the industry in US. The chemical industry has not been a major part of this discussion. So, in this study the chemical industry will be considered for a thorough analysis of the energy use and energy management activities. A general background of the chemical industry will be discussed in the following paragraphs followed by economic profile and trends in that industry.

\subsection{Chemical Industry}

The chemical industry is one of the keystones of the U.S. economy. It converts raw materials such as oil, natural gas, air, water, metals and minerals into more than 
70,000 different products. There are very few goods that are manufactured without some input from the chemical industry. Chemicals are used to make a wide variety of consumer goods, as well as many other products that are essential raw materials to agriculture, manufacturing, construction, and service industries. The chemical industry itself consumes 26 percentage of its output [35]. Major industrial customers include rubber and plastic products, textiles, apparel, petroleum refining, pulp and paper, and primary metals. Major chemical products are industrial inorganic chemicals, plastics and rubbers, drugs, soaps, detergents, cleaning preparations, perfumes, cosmetics, paints, varnishes, and allied products, industrial organic chemicals, and agricultural chemicals.

The U.S. chemical industry is the world's largest producer of chemicals in the \$1.5 trillion global enterprise. It produces over 25 percent of the total production worldwide and had a record trade surplus of $\$ 19.2$ billion in 1997 . The industry continues to grow with profits reaching an all-time high of $\$ 44.8$ billion in 1997 . There are 170 chemical companies with more than 2,800 facilities abroad and 1,700 foreign subsidiaries or affiliates operating in the United States [35]. More than a million people are employed in the industry. Over half of the industry employees are production workers earning weekly wages that are 30 percent higher than the manufacturing average. The chemical industry is one of the largest U.S. private sector investors in research and development, with chemical patents accounting for 15 percent of the total awarded in the United States [35]. The chemical industry is the second largest consumer of energy in manufacturing and spends over $\$ 5$ billion annually in abatement of pollution. The following table (Table 1.1) shows the economic and trade statistics of the chemical industry in 1997.

\begin{tabular}{|l|l|}
\hline Value of shipments & $\$ 392.2$ billion \\
\hline Employment & $1,034,000$ \\
\hline Average hourly wages (production workers) & $\$ 16.6$ \\
\hline Capital expenditures & $\$ 25.4$ billion \\
\hline R \& D expenditures & $\$ 18.7$ billion \\
\hline Pollution abatement expenditures & \\
\hline Capital & $\$ 2.1$ billion \\
\hline Operating & $\$ 4.3$ billion \\
\hline
\end{tabular}




\begin{tabular}{|l|l|}
\hline \multicolumn{2}{|l|}{ Trade } \\
\hline Imports & $\$ 50.3$ billion \\
\hline Exports & $\$ 69.5$ billion \\
\hline
\end{tabular}

\section{Table 1.1: Economic and Trade Statistics of the Chemical Industry in 1997 [1]}

The chemical industry continues to be a strong contributor to the U.S. economy, with shipments increasing by more than 5 percent every year over the last decade. Pharmaceuticals is the highest valued sector (24 percent of shipments), followed by organic chemicals at 21 percent and plastics at 16 percent. The following table (Table 1.2) shows the export value in 1996 [35].

\begin{tabular}{|l|l|}
\hline Pharmaceuticals & 86.5 \\
\hline Organics & 75.7 \\
\hline Plastics & 59.6 \\
\hline Soaps / Cleaners & 51.8 \\
\hline Inorganics & 27.7 \\
\hline Misc. & 24.5 \\
\hline Agri-chemicals & 23.4 \\
\hline Paints & 18.3 \\
\hline
\end{tabular}

Table 1.2: Billion \$ in Shipments in 1996 [35]

The chemical industry produces 70,000 chemical products in 12,000 plants, with 95\% in batch operations. Organic chemicals accounted for the greatest share of production by weight in 1997. The top five organic chemicals include ethylene, propylene, ethylene dichloride, methyl-tert-butyl-ether (MTBE), and vinyl chloride. The top inorganic chemicals produced are nitrogen, oxygen, chlorine, and sodium hydroxide. Major agricultural chemicals used in the production of fertilizers are ammonia and sulfuric acid. The following table (Table 1.3) shows the annual production of the various chemicals in 1997. 


\begin{tabular}{|l|l|}
\hline Chemical & Production in 1997 \\
\hline \hline Top 50 chemicals & 364.2 million tons \\
\hline Organic & 141.7 million tons \\
\hline Inorganic & 102.4 million tons \\
\hline Agricultural chemicals & 44.9 million tons \\
\hline
\end{tabular}

Table 1.3: Annual Production in 1997 [35]

Most of the production of the basic chemicals in the United States is concentrated along the Gulf Coast, where petroleum and natural gas feedstocks are available from refineries. About 70 percent of all primary petrochemicals are currently produced by Texas and Louisiana. Production of other products is widely dispersed among the states.

\subsection{Energy Usage in Chemical Industry}

In a chemical industry, the energy is used to supply heat and power for manufacturing processes and also for energy feedstocks that are used as raw materials to produce a range of products. Also, certain chemical, physical, and biological separation and synthesis processes are required to transform the feedstocks into the final products, which consume enormous amounts of energy in the form of heating, cooling, or electrical power.

The industry uses a diversity of fuel sources for its energy needs with nearly 50 percent of the total used as feedstocks. The chemical industry is the largest consumer of natural gas requiring more than 10 percent of the total domestic energy usage and nearly 7 percent of all petroleum products consumed in the United States. Energy expenditures represent a significant portion of manufacturing costs in the chemical industry. Within industrial segments, energy usage is closely related to product configurations and whether the energy is used as feedstocks. Inorganic chemicals are not usually made from energy feedstocks, but are made primarily from mineral ores taken from the earth. Organic chemicals begin with feedstocks and the manufacturing plants are usually located close to petroleum refineries to ensure reliable supply of these materials. Availability of feedstock is a primary concern for many chemical producers and the industry is highly susceptible to volatility in price and supply of energy feedstocks. 


\subsection{Demographics of Energy Usage}

According to the Manufacturing Energy Consumption Survey (MECS), the U.S. chemical industry consumed about 5.3 quadrillion Btu of energy in 1994. This represents about 7 percent of domestic energy use and about 25 percent of all U.S. manufacturing energy use. The cost of energy purchases was about $\$ 18$ billion in 1994, which is about five percent of the value of shipments that year [3].

The industry is the largest single consumer of natural gas (over 10 percent of the domestic total) and uses almost all the liquefied petroleum gas (LPG) consumed in the U.S. manufacturing industry. Nearly all the LPG and about one fourth of the natural gas are used as feedstocks. Other sources of energy include byproducts produced onsite, hot water and purchased steam. The pie-chart (Figure 1.4) shows the energy usage by fuel in the industry in 1994.

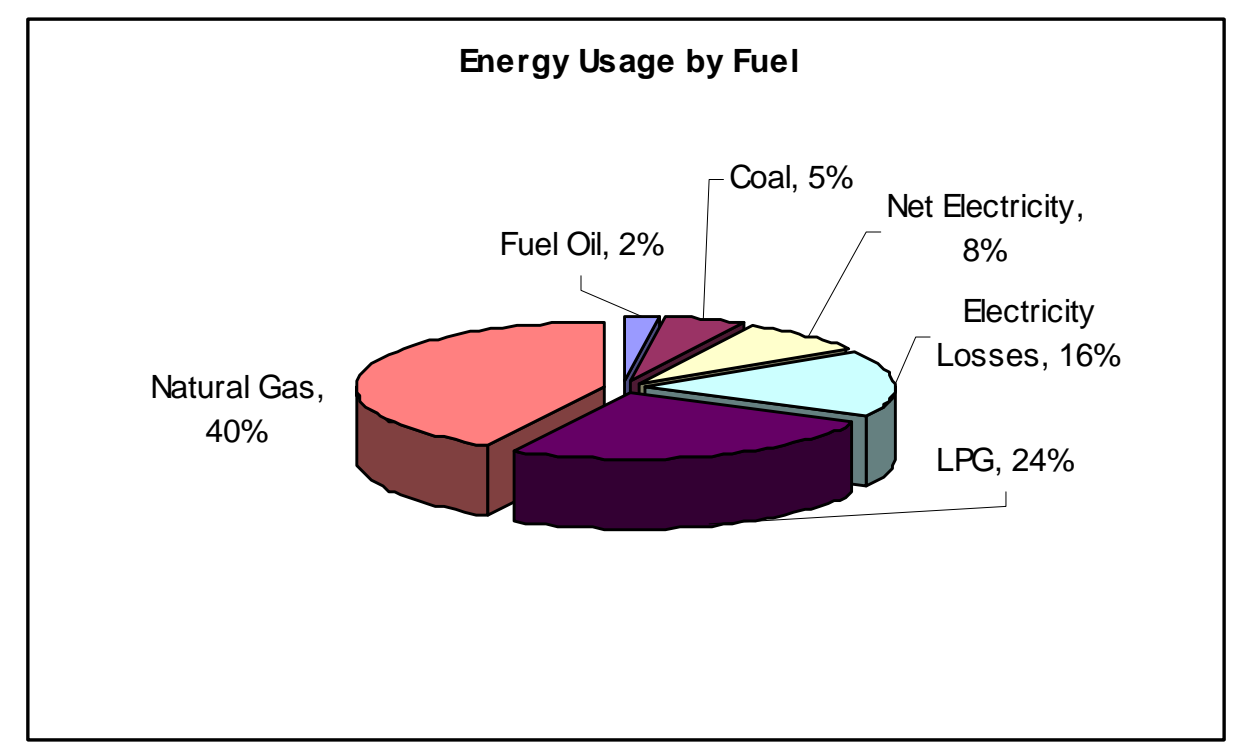

Figure 1.4: Energy Usage by Fuel in 1994 [3]

Nearly 50 percent of the energy used in the industry is transformed into chemical products. The largest use of energy for heat and power is in boilers (43 percent) to produce steam to drive chemical reactions and perform product separation and finishing operations. About 27 percent of the energy use in the industry is used for process heating and cooling. Electricity is used to power equipment and drive electro chemical processes, and also to heat, light and cool the industry facilities. The following chart (Figure 1.5) shows the energy for heat and power consumption in 1994. 


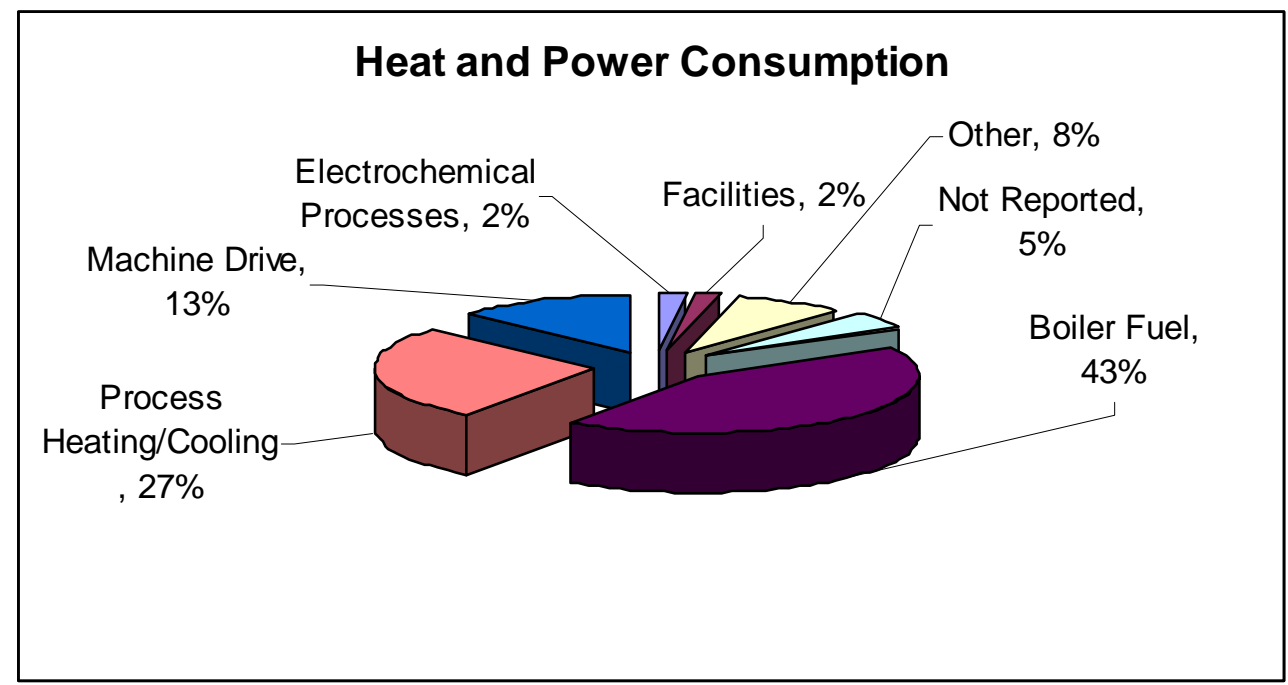

Figure 1.5: Heat and Power Consumption of Energy in 1994 [35]

Organic chemicals have the highest energy requirements of all the chemical sectors. It accounts for about 49 percent of the total industrial energy use. More than 50 percent of the energy consumed in organic chemicals manufacture is in the form of feedstocks, that is natural gas and LPG. Inorganic chemicals require little or no feedstock energy as they are made from ores, air and water. Energy costs account for up to 85 percent of the production costs for some chemical products. The chemical industry spent about \$18 billion in 1994 on energy, which constituted about 26 percent of all energy expenditures in the manufacturing sector. The following chart (Figure 1.6) shows the energy expenditures in billions of dollars for 1994 [35].

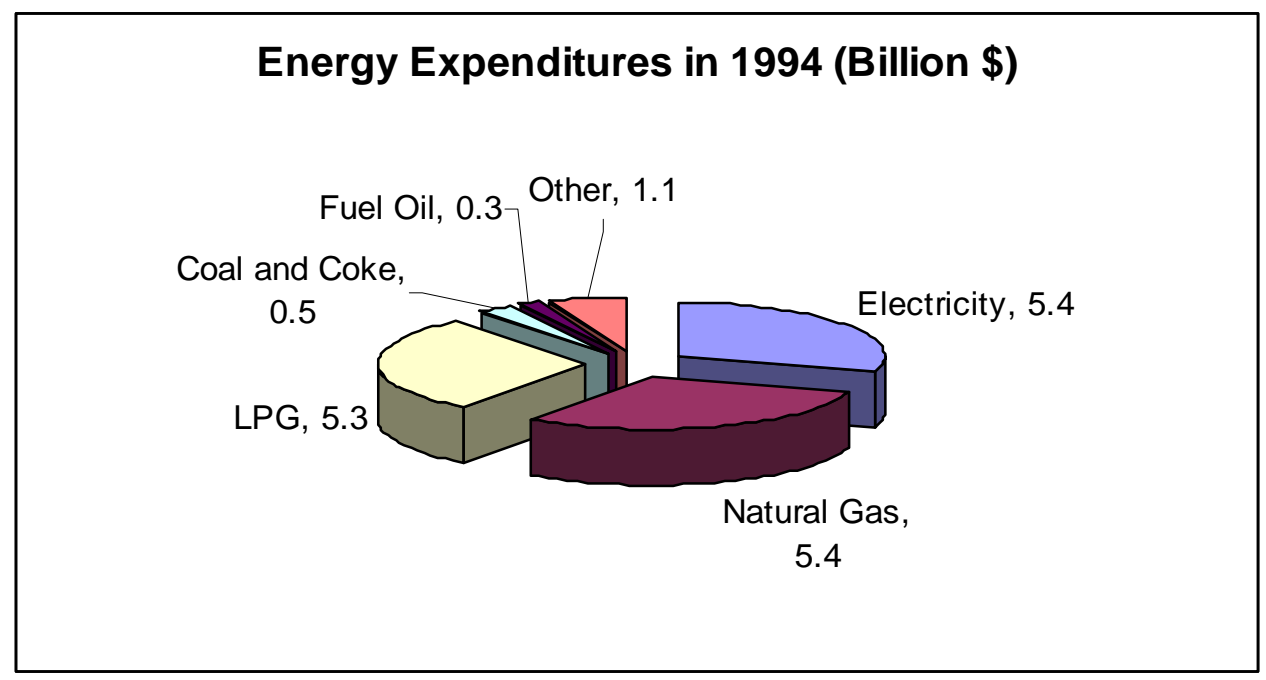

Figure 1.6: Energy Expenditures in 1994 (Billion \$) [35] 
About one third of electricity used by the chemical industry is produced onsite, mainly by cogeneration. Cogeneration can provide competitive advantages over purchased electricity as the thermal efficiencies are much higher and excess electricity can be sold back to the grid. The cogeneration capacity has been steadily increasing in the chemical industry. The following chart (Figure 1.7) shows the net demand for electricity.

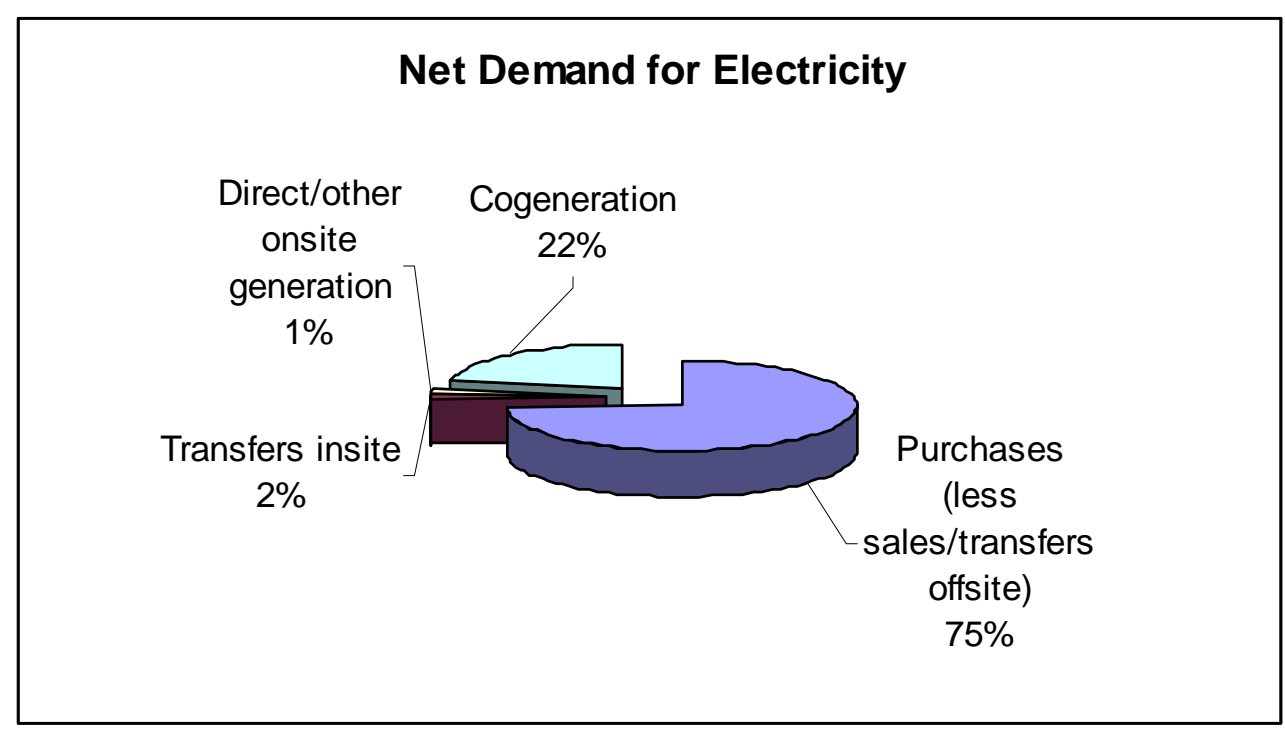

Figure 1.7: Net Demand for Electricity in 1994 [34]

There have been significant improvements in the energy efficiency over the last two decades in the chemical industry. Fuel and power energy consumption per unit of output declined by over 39 percent after the 1973 oil crisis, as a result of aggressive energy management and housekeeping programs. Improved process and equipment designs and adoption of energy-efficient practices have also helped to reduce the overall energy intensity. The following figure (Figure 1.8) shows the total energy usage. The OIT's energy intensive industries account for about two-thirds of all energy used in U.S. industry. From the figure it can be seen that the chemical industry is the second largest consumer of energy next only to petroleum refining. 


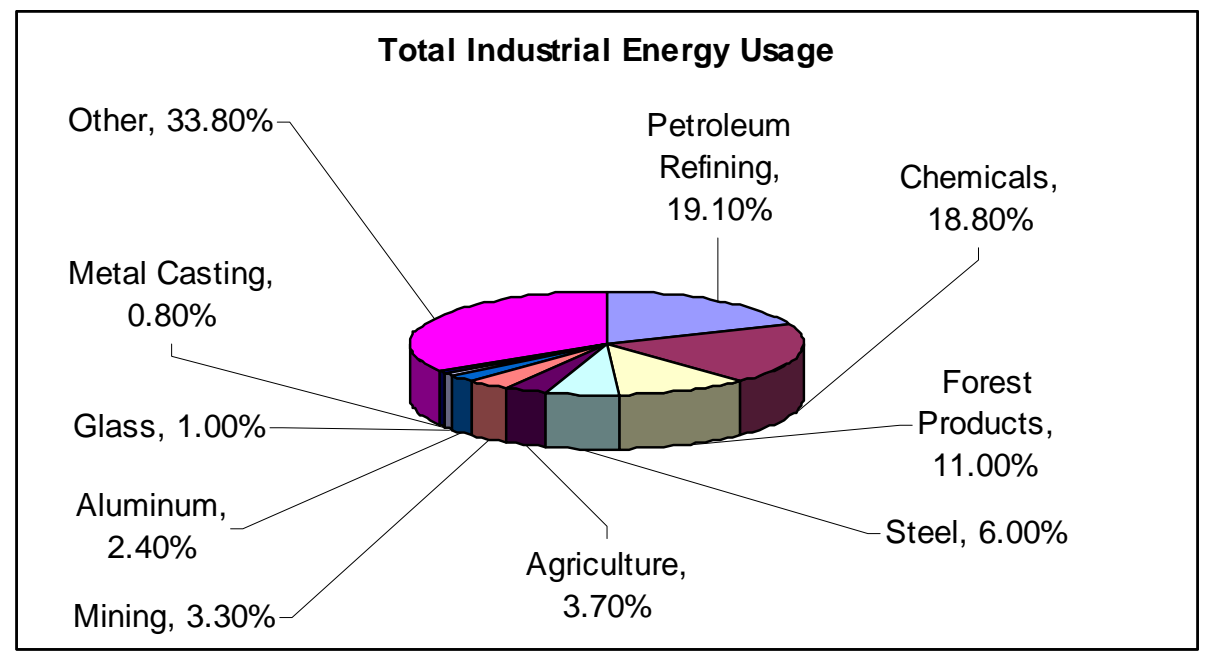

Figure 1.8: Total Industrial Energy Usage [34]

\subsection{Energy - Intensive Processes}

Chemical industry is the second most energy-intensive industry in the U. S. manufacturing industries. This is due to the fact that energy is required in the process, in addition to the normal industrial uses of energy. The six most energy-intensive processes in chemical manufacturing are [36]:

1. Fuel - heated reaction

This involves all the processes that require some type of heat to force a chemical reaction to take place. The various energy sources include steam, natural gas, residual oil, distillate oil, and also, coal combustion.

2. Refrigeration

It includes all the processes that compress and expand a refrigerant, such as ammonia or a fluorocarbon, for the purpose of cooling feedstocks or products below ambient temperatures.

3. Machine drive

Many of the processes use machine drives to pump, compress, or move feedstock and end products. Machine drives arise from electric motors, steam turbines, or gas turbines. Mixing and blending are the most energy intensive processes due to the high viscosity of the materials.

4. Distillation

It includes all the processes that require physical separation of end products from both feedstocks and byproducts by evaporation and condensation. 


\section{Electrolysis}

This includes all the industrial electrolytic processes in which electricity is used in direct chemical conversion.

\section{Evaporation}

It includes those processes that use passive evaporation cooling. Usually the evaporated water is lost to the atmosphere and the heat energy involved is unrecoverable.

\subsection{Need for Energy Assessments in Chemical Industry}

The keystone to any energy conservation program is the energy assessment [11]. Industrial energy assessments or audits refer to a detailed analysis of existing operations in a plant in order to improve productivity. They serve to identify the various energy streams in a facility, to quantify energy use and to identify the various conservation opportunities. It is a brief onsite survey and analysis of a facility, its energy usage patterns, identification of opportunities for saving energy through implementation of operating and maintenance changes and the economics in the implementation of the energy conservation measures.

An energy assessment can be considered similar to the monthly closing statement of an accounting system. One series of entries consist of the amount of various streams of energy consumed during the month. The second series lists how the energy was used in various processes like lighting, heating, air conditioning, boilers, motors, pumps and others.

The previous section (section 1.8) discusses by the energy intensive nature of the chemical plants. The energy is used for the normal industrial uses and also in the process itself. The various energy users are huge in size and capacity, and are used year round to keep the plant operational. Hence, even the normal recommendations that are applied in other industries would realize huge savings in chemical industries. Since most of the chemical plants have a large number of energy users, it will be beneficial if there is a methodology for performing the energy assessment. This methodology can be applied to any large plant and would help in realizing the maximum benefit. 


\subsection{Industrial Assessment Center (IAC)}

Intelligent usage of energy improves productivity, saves money and is good for the environment. Energy can account for about ten percent or more of an industry's total operating costs [8], depending on the industry. Yet, most of the facilities lack the resources and the expertise in-house to systematically analyze and identify the conservation opportunities of their own. This problem is overcome by the Department of Energy's (DOE) Office of Industrial Technologies (OIT) sponsored program to conduct no-cost energy assessments since 1978. These assessments are performed by University centers across the country by team of engineering faculty and top students. These centers called Industrial Assessment Centers (IAC) conduct energy assessments, and productivity and waste reduction analyses.

In order to be eligible for an IAC assessment, the industry has to be a small or medium-sized plant in the standard industrial codes of 20 to 39. They should be located within 150 miles of one of the centers. They should also have gross annual sales less than $\$ 75$ million, have fewer than 500 employees, have no in-house technical staff to perform these analyses and have annual energy bills less than $\$ 1,750,000$. If any three of the four criteria mentioned are satisfied, then the plant qualifies for a free energy assessment.

On an average, the energy saving recommendations (ECO) from each IAC assessment result in annual energy savings of about 4 billion British Thermal Units (Btus) and identify annual cost savings of about \$40,000 [41]. By the end of 1994, more than 5,000 assessments had been performed, cumulatively saving 94 trillion Btus and decreasing the emissions of harmful greenhouse gases by 200,000 metric tons of carbon equivalent. The return on the investment is excellent on the government spending of \$27 million on the IAC program with the industrial savings at more than $\$ 500$ million.

\subsection{Typical Process in an IAC Audit}

In a typical Industrial Assessment Center (IAC) audit, the directory of manufacturers is used to select the list of industries that satisfy the basic criteria required for performing an assessment as set by the U.S. Department of Energy (DOE). The companies are also selected based on support from the utilities and referrals. The companies are called-up and described about the IAC program and for their approval for 
an assessment. Upon confirmation of the assessment date, the company sends information on the utility usage data along with information on major energy using equipments, building information, process details, safety requirements and the potential energy savings recommendations (ECO). The information sent by the company is reviewed for completeness.

Upon arrival at the plant at the scheduled date, a meeting is held with the management and the IAC program and the DOE efforts are explained. Any additional information required for the data sent earlier by the industry are collected. Then the assessment team goes out on a plant tour starting from the point of entry of raw materials to the shipment area. The various processes are reviewed in the order of production as explained by the plant personnel. Upon completion of the tour, the team huddles together by themselves to discuss about the various suggestions, to compile a list of potential ECO's and to make assignments for the data collection. Then the team goes out for each of the ECO and collects the required data with help from the plant personnel, using the appropriate instrumentation. Upon completion of data collection, the potential ECO's are discussed with the management and the team starts back.

Once back from the assessment, the ECO's are analyzed and calculations are done to produce a final report to the industry within six to eight weeks. The plant data along with the various ECO's and the energy and cost savings are uploaded to a national database for the development of Best Practices. The uploaded data does not reveal any proprietary information about the industry and identifies it only by the Standard Industrial Code (SIC), their principal product, and the plant city and zip code. Implementation reports are obtained from the industry after nine months of submitting the final report. This report has information about the energy and cost savings after implementation of each of the ECO’s along with the actual implementation cost.

\subsection{Why Typical Process may not be enough}

The typical energy assessment procedure requires a visual inspection of the facility to determine maintenance and process energy saving opportunities, tests and measurements to quantify the energy usage and losses and determine the economics for changes. Only when the energy problems faced in the plant are reviewed in a total 
perspective, do the solutions and the various conservation opportunities become visible. This is called the systems engineering approach. In a systems approach, the systems are evaluated macroscopically first and then micro studies are initiated only within the framework of macro study to ensure the effectiveness of the assessment [9]. The systems engineering approach deals with the analysis of an unsystematic mess and replacing it with a more systematic structure or system [39]. It will be more advantageous to consider complex systems as systems. A system is a complex grouping of resources in the form of men, machinery, materials and money. It can be broken down into its sub-systems that are useful smaller units of the main system, after careful and thorough analysis of the problem. The performance of an individual unit interacts with the performance of the other units in the system and hence, cannot be designed in isolation of the other units. The system has a hierarchy of sub-systems in which the units higher in the hierarchy exert a considerable influence on the performance of the system that is studied. In order to function efficiently, a system must be designed to achieve its overall objective in the best possible way.

Systems engineering is the activity of planning, designing, constructing, checking and operating complex systems. It helps in designing the best system for the given objective. The various stages in the development if a systems engineering project are systems analysis, systems design, systems implementation and systems operation. The systems engineering approach starts with an analysis of the system and how it can be improved to overcome the problems faced currently. It identifies the system and all it sub-systems and defines objectives. Then various ways of operating the future system are evaluated and the best way is identified based on defined criterion of performance. The results of the system study are presented to the management and upon approval the recommended system is implemented. The system is then handed over to the operating managers and is monitored to ensure that the system is performing as intended.

The systems approach recognizes that each system is an integrated whole even though composed of diverse, specialized structures and sub-functions. It further recognizes that any system has a number of objectives and that the balance between them may differ widely from system to system. The method seeks to optimize the overall system functions according to the weighted objectives and to achieve maximum 
compatibility of its parts [40]. The systems approach emphasizes the ideas that are common to the successful operation of somewhat independent parts in an integrated whole system.

In a systems approach, both the supply and demand sides of the system are analyzed and their interactions are studied. The supply and demand sides of a manufacturing facility do not work independently of each other [37]. The focus is shifted from the individual components to the total system performance. This is an easy method to optimize efficiency choices. Each component in the system can be designed to achieve greater efficiency as a whole rather than as individual components. It helps to avoid unfavorable systems interactions and to achieve the correct balance of cost reduction and other considerations such as plant productivity and systems reliability.

Also a typical energy assessment is usually completed in a day. This does not allow for the complete review of the plant's energy usage patterns for various individual processes. For a complete understanding of the energy usage, the plant and the processes have to be analyzed and monitored over an extended period of time.

\subsection{Systems Approach}

The systems approach to the manufacturing process is illustrated by Figure 1.9. The utilities, raw materials and labor are the input to the manufacturing processes. The various operations are performed in the manufacturing process and the finished product is the output. The output is analyzed to verify whether the results are as per the requirements. If not, modifications are done to the input or to the manufacturing process to obtain the desired output. If the output obtained is satisfactory, continuous improvements are done to maintain optimal efficiency of the processes.

The first important step involved in a systems approach to plant-wide energy assessment is to determine how energy is used in each process and account for consumption and costs, and can be initiated by a brainstorming session with plant engineers and operators. As the next part, the energy consumed in any form of energy or utilities at the plant level must be studied. Energy performance of each of the consuming equipments like boilers, furnaces, cooling towers, compressors, heat exchangers, pumps, motors, turbines and piping networks, must be evaluated. 


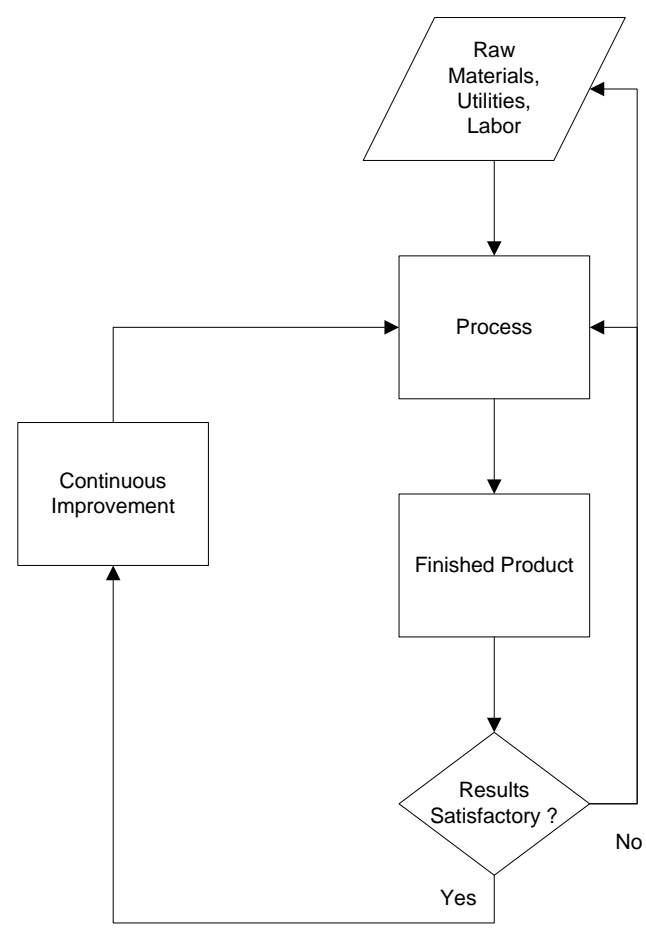

Figure 1.9: Systems Approach

Establishing an efficient process for system management begins with a complete assessment and analysis of demand, storage, distribution, and supply [37]. The first step is to conduct a comprehensive assessment, which examines the system performance and condition of the equipment from point-of-use to the supply. Once the assessment is completed, benchmarks and performance standards should be established. The next step is to match the capacity to demand. The last phase in gaining and maintaining system efficiency includes ongoing maintenance and continuous performance measurement. A system-wide assessment should be performed at regular intervals and also at times of specific change.

A detailed plant-wide energy assessment attempts to balance the total usage of energy with the various energy inputs. It serves to quantify the energy usage and loss using a systems approach and tries to determine the various ways to reduce the energy consumption per unit of end-product and to lower the operating costs. It helps in effectively managing the various users of energy at minimum energy costs and arriving at rewarding investment decisions. 


\subsection{Plant-wide Energy Assessments}

Plant-wide assessments help a manufacturer to take a comprehensive look at the energy usage through the various plant operations. They evaluate opportunities for improvements in industrial energy efficiency, waste reduction, productivity, and competitiveness. The plant-wide assessments are cost-shared by the Department of Energy (DOE) and they use process engineering and best practices analysis techniques. For a relatively low initial investment, these plants can expect to realize a minimum of $\$ 1$ million in savings annually from energy costs, waste reduction, and increased productivity [8]. The assessments evaluate a variety of energy conservation opportunities in steam systems, electric-motor systems, compressed air systems, heat exchange networks, cogeneration opportunities, and other areas. The assessments evaluate energy efficiency opportunities using process engineering and best practices techniques. The goal of plant-wide energy assessments is to develop a comprehensive strategy that will significantly increase plant energy efficiency and reduce environmental emissions [10].

The plant-wide energy assessments would provide an opportunity for the industries to identify the various energy consumers in the manufacturing facility and to quantify them to improve the energy efficiency, reduce energy consumption, and improve the process and manufacturing efficiency. The goal of Office of Industrial Technologies (OIT) of DOE is to reduce the environmental emissions. The OIT's involvement is to provide cost-sharing funds for the assessment and to offer technical assistance to the assessment teams through the Best Practices initiative of the Industries of the Future (IOF) program. The nine energy-intensive industries included in the IOF program are forest products, chemicals, petroleum refining, steel, aluminum, metal castings, glass, mining, and agriculture. These industries use more than 80 percent of the total energy consumed in all the manufacturing industries in the United States.

The various teams that perform the assessments give consideration to the adoption of the best available and emerging technologies using a variety of tools, information, process engineering techniques, and Best Practices plant support. The initial step involved in conducting a plant-wide energy assessment is to initially conduct a survey of the entire plant's energy requirements and profile the various energy-intensive processes. The next step involved is to focus on the assessment of those energy systems that would 
offer the largest cost savings and return on investment. The plant-wide energy assessments help the industries to use the experiences from the assessment to implement projects to replicate similar projects at other plants within the company, or to other companies that have related applications.

\subsection{Systems Approach to Plant-wide Energy Assessments}

Normal one-day energy assessments look at each and every area for energy saving recommendations based on heuristics. This will not be feasible in a huge manufacturing plant. For example if there are around 1000 pumps in a plant, it is beyond the ability of the plant personnel to monitor all the pumps to identify the few where energy efficiency opportunities are possible. However, if appropriate system assessment methodologies are applied, the systems that offer the largest opportunities can be identified quickly.

The normal IAC approach for assessments is good for small and medium-sized facilities, but is not good for large facilities. This is because the IAC assessments usually look for only the low-hanging fruits, that is, they look at only the opportunities which would give immediate payback on the investments involved. They look at only the normal ECO's in the areas of lighting, steam, compressed air, motors and productivity. Energy saving recommendation that involve high implementation costs is not done. Also, it is not possible to perform assessment on a large plant in one day as done in the IAC assessments, as the utilization of the energy in the plant is spread over a large area of the plant. In large plants, the assessment team has to incorporate the use of the knowledge and experience of the plant personnel to look for the various problem areas and methods for improving them efficiently.

Plant-wide energy assessments can use systems approach to arrive at the desired efficiency of processes and in identifying the most promising energy efficiency opportunities in the process and utility applications in the plant. A systems approach is necessary for energy assessments as the manufacturing is spread over various parts of the plant and the utilities serve the manufacturing activities in a non-concentrated manner. It will not be feasible to look at each and every area in a plant for energy saving recommendations. Criteria and metrics have to be developed from the initial preassessment data collected. Priorities have to be made to look at the major users of energy. 
This aspect of the system approach helps in focusing on the major energy saving opportunities in the plant and arrives at the maximum energy and cost savings.

\subsection{Research Objectives}

The objectives of this work are to develop a standardized methodology for conducting plant-wide energy assessments in chemical plants using the collected data, determine energy conservation opportunities and recommendations. If there is no standardized methodology, the assessor will get overwhelmed with the amount of data in a large facility and might focus on insignificant areas for energy savings. The specific objectives of the research are listed below:

1. Develop a methodology for performing plant-wide energy assessments using a systems approach.

2. Showcase the methodology with respect to the data collected in a chemical plant that was assessed.

3. Perform a sensitivity analysis on the key factors governing the energy savings potential.

The methodology serves to standardize the way the assessment is done by using a systems approach, which helps in identifying the best possible savings in industry. This assessment methodology is then guided through by the various experiences obtained in a plant-wide energy assessment done. This step helps to illustrate the process with much

more detail and helps in better understanding of the methodology. The sensitivity analysis that follows this step helps in identifying factors that have a better impact on the energy savings potential for some of the areas analyzed.

\subsection{Conclusion}

This chapter helps to understand the magnitude of energy consumption in industry and the need for better energy conservation. The typical energy assessment approach is described for an IAC audit. In order to increase the energy savings potential, a systems approach should be used to perform the plant-wide energy assessments. A standard methodology is required to perform these assessments in order to realize the best possible results. The next chapter discusses about the research work performed in this field in literature. 


\section{Literature Review}

Energy assessment means different things to different individuals. According to energy auditor's handbook [7], the energy audit is a brief onsite survey and analysis of a plant, its energy usage patterns, identification on opportunities for saving energy through implementation of operating and maintenance changes, and an assessment of its need for implementation of energy conservation measures. The following sections discuss the publications in the field of energy assessments.

\subsection{Energy Conservation}

In order to meet the increasing demand for energy at a reasonable cost with minimized effects of energy production on the environment, the pacific northwest region pursue energy conservation as a source of energy [13]. Since the conservation program was started in 1987, over 52 energy savings plan projects have been completed for a combined annual savings of more than 98 million kilowatt - hours per year, or 11.2 average megawatts.

One of today's primary requirements for lighting is energy conservation [14]. Lighting is the target for the energy and environmental conservation measures as it consumes around 25 percent of the total electricity used in the business world. This article discusses about the utilization of innovative light sources in various applications.

The implementation of energy efficient technologies among manufacturing firms is analyzed in [16]. It examines the factors responsible for the success or failure of the implementation process. It discusses about the various barrier studies and results of surveys on energy assessments performed that will make major barriers apparent and allow the formulation of strategies that favor implementation of energy-efficient technologies.

The goal of efficient boiler operations is to get all the possible energy from the fuel into the working fluid of the boiler. This saves money, produces higher boiler productivity and reduces pollution. Some of the basic but overlooked methods for improving boiler efficiency are discussed in [19]. It reviews the various boiler plant 
losses and the ways to promote higher efficiencies in spite of the ongoing responsibilities of operations, pollution abatement and various other challenges.

Opportunities to promote cost-effective energy efficiency in the Texas oil and gas production industry is explored in [21]. Specific energy efficiency measures are identified and potential energy savings are quantified.

U. S. manufacturing has a high per capita consumption of energy in the industrial sector and hence, the highest percentage of productive energy has to be extracted from each Btu of energy produced. The design and development of a computer based tool (ENERGEX) which aids the industrial user in developing energy conservation opportunities in plants is described in [22]. The expert system offers expert advice in various areas including power factor improvement possibilities.

A software program to assist manufacturers in minimizing waste and improving energy efficiency that was developed in the University of Maine is discussed in [25]. The software combines the most common recommendations made in the U. S. Department of Energy's Industrial Assessment Center program in a user-friendly package that can be used by computer novices. It also includes "wizards" that serve as an expert system to lead the user through an assessment of their total facility.

The nature of energy conservation opportunities that exist in glass manufacturing facilities is described in [29]. The most significant recommendations are related to the insulation of heat generating surfaces, preheating the combustion air in boilers, and heat recovery and distribution. This article discusses about the tools and the assessment methodologies.

\subsection{Energy Assessments in Industries}

The methodology for conducting an energy assessment that attempts to conduct a comprehensive look at the energy utilization of all forms is discussed in [12]. It addresses the issue that many of the energy assessments conducted by companies have the potential to miss substantial energy conservation opportunities. It describes a methodology for conducting an energy assessment that will make efficient use of the utility's and customer's time. 
The feasibility of developing a self assessment tool for energy management and conservation is investigated in [15]. It develops a simple tool that can be used by plant personnel. The tool developed consists of a benchmark or reference point for energy use as well as a set of possible energy conservation opportunities. Regression analysis and an estimate of standard deviation are used to establish a reference point.

The instrumentation needed to facilitate a chiller plant energy efficiency monitoring that is a prerequisite to any efficiency improvement initiatives for centrally air-conditioned buildings is discussed in [17]. It describes how the plant performance was estimated based on limited data that was available.

A self assessment tool for energy management in the cardboard industry (SIC codes 2652 and 2653) is discussed in [18]. Using data from 25 companies, a model is obtained for calculating a reference point for energy usage. The actual energy use for a company can be compared with this reference point and if energy conservation is beneficial, a set of energy conservation opportunities should be explored.

Valid energy efficiency improvement estimates require energy assessments. Important information on starting an energy assessment is discussed in [20]. Energy auditing is the first phase in improving the energy efficiency of a facility. Preparing the energy management report, implementing and monitoring are the other three phases. It discusses the purpose of conducting an energy assessment and how to attain a successful energy assessment program. Pre-site, on-site, and post-site efforts are described. It also includes information for conducting lighting energy assessments. The importance of interaction among energy conservation measures is emphasized.

Results of pre-investment surveys that were conducted at four commercial airconditioned facilities in India in 1995 are presented in [23]. These building were chosen because as centrally air-conditioned buildings, they are some of the most energyintensive in the commercial sector. The survey team's findings confirmed that commercial air-conditioned buildings have great investment opportunities in energy conservation.

Energy engineers focus primarily on energy dollar savings from the installation of demand-side energy management projects. There is also an environmental benefit from projects which result in the reduction of fossil fuel consumption as discussed in [24]. This 
article presents five cases of energy projects which were worked on by the author. These projects resulted not only in energy and cost savings, but also in over 110 thousand tons of pollution avoided each year.

A rational approach to targeting energy costs and to outline the key strategies in the assessment of iron foundries are discussed in [27]. Large metal-casting foundries are excellent targets for energy savings opportunities and should be targeted for continuous technological improvements.

An extended assessment of a manufacturing plant is described in [28]. The assessment was extended by adding monitoring of selected electrical systems in the plant with portable data logging equipment and identifying possible assessment recommendation for alter investigation. Monitoring identified three recommendations about equipment being left on that likely would not have been identified during a usual assessment, and provided data for quantification of savings.

Higher productivity and lower operating costs can be achieved along with many other benefits by performing a plant energy assessment as evident from [32]. A producer of athletic clothing performed a plant energy assessment on its steam, motor, and compressed air systems for improvement opportunities at their largest and most integrated manufacturing plant. Implementation of the opportunities realized in the assessment increased the throughput capacity by 37 percent while reducing the energy needed per pound of product by 38 percent, saving \$165,000 annually in fuel expenditures. It is found that the benefits of an energy assessment are not limited to utility and fuel cost savings, but also include improved productivity, increased equipment life, decreased risk of financial penalties, and increased order turnaround. Environmental emissions and penalty risks are also generally improved with energy efficiency projects.

The systems approach to an efficient compressed air management is explained in [37]. Optimum operating benefits are obtained when air systems are considered as a whole and not as individual items. The supply and demand sides in a manufacturing facility do not work independently of each other. They work together as a system and that is how they should be analyzed, monitored and controlled. 


\subsection{Energy Assessment in Chemical Industry}

The process of identifying opportunities for energy conservation in plants is described in [26]. It also discusses the recommendations made to plant managers at some of the plants visited including a glass manufacturing plant, chemical plant, and alumina plant and several others. It deals with cogeneration opportunities in the chemical plant using a byproduct from the process in the plant. It also provides an update on the implementation plans for some of the recommended energy efficiency measures at these industries.

The energy management program in a specialty chemical manufacturer is discussed in [30]. The program has resulted in a 17 percent reduction in energy use on a per pound of product basis, saving 3.25 trillion btu's and \$15 million each year. This article discusses this program, its history, successes, and the unique characteristics that have contributed to the successes. One of the key factors in the success of the program was the plant's willingness to work as a team within the various business units in the plant.

The strategy used in a chemical plant to reduce energy consumption is discussed in [31]. The plant identified, designed and completed several projects to improve its chemical production processes. These projects reduced steam usage, fuel gas usage, and electricity usage. Though there was capital investments involved with few of the projects, lot of the projects used the existing assets more efficiently. The plant realizes cost savings by operating the plant more efficiently.

\subsection{Efforts of U. S. Department of Energy}

Industries consume around 36 percent of all energy used in the United States [34]. The U. S. Department of Energy's Office of Industrial Technologies (OIT) has established a cooperative program with industry known as the "Industries of the Future" (IOF) or "Vision Industries" [33]. These industries include agriculture, aluminum, chemical, forest products, glass, metal casting, petroleum refining, and steel. Collectively, these energy- and technology-intensive industries produce $\$ 1$ trillion in annual shipments, account for 5 percent of the GDP, and supply more than 90 percent of the materials used in finished products. 
As part of this program, the industries have developed strategies to forecast growth and identified the tools that are needed to increase their competitiveness. The strategies address various issues from production techniques to utility costs including energy. Through the IOF partnerships, OIT provides about $\$ 150$ million annually to support around 600 active projects. These projects involve over 2,000 partners engaged in research and development, technology demonstrations, and BestPractices activities.

BestPractices offer a wide range of technology solutions for industrial processes and plant-wide operations. BestPractices’ plant assessments help manufacturers identify the best available opportunities to save energy and to cut costs. Plant-wide energy assessments assist in developing a comprehensive strategy for increasing efficiency, reducing emissions, and boosting productivity for large manufacturers. Funding of up to $\$ 100,000$ is available for each project selected [45], with a required industrial cost share of at least 50 percent through a competitive solicitation process.

Plant-wide energy assessments identify overall energy usage in manufacturing plants and suggest opportunities for reducing energy consumption and the operating costs. The findings from a single assessment can be replicated in at least seven other facilities with equivalent systems and energy usage patterns. For a relatively low initial investment, companies that participate in the assessments can expect to realize a minimum of $\$ 1$ million in savings annually from energy costs, waste reduction, and increased productivity [44]. The implementation of the projects has a payback usually of less than 18 months. The results, successes, and experiences from these assessments are published in case studies, encouraging other manufacturers to adopt and implement similar approaches for increasing energy efficiency and reducing environmental emissions. Confidentiality is protected and proprietary company information is released.

The plant-wide assessment at a pulp and paper mill identified opportunities to recycle effluent streams, which reduces the need for process steam, fresh water, and energy to cool the effluent. An assessment at an aluminum extrusion plant identified eight areas for more analysis. Energy saving opportunities included improved heat recovery, furnace operations, and development of process-energy use targets. The assessment at a plant making technology-intensive metal products resulted in 12 separate projects, including improvement to their process heating and compressed air systems. 
From the year since OIT began the plant-wide assessments in large plants, over 32 plants had submitted proposals, and 13 assessments have been completely awarded. In the first five assessments that had been completed individual third-party assessment teams had documented potential energy and related cost savings of \$18 million.

\subsection{Conclusion}

The above literature survey gives an idea of the work done in the field of energy assessments and the efforts of the DOE. Many issues related to energy conservation and energy assessments in industries are discussed. But there is very little literature found on the methodology for a systems approach to plant-wide energy assessments in chemical plants. Most of the research is on energy assessments for individual areas in a plant. Research in the area of a methodology for a systems approach to plant-wide energy assessments and sensitivity analysis would be of immense help to the chemical industry and also the nation. 


\section{Plant-wide Energy Assessment Methodology}

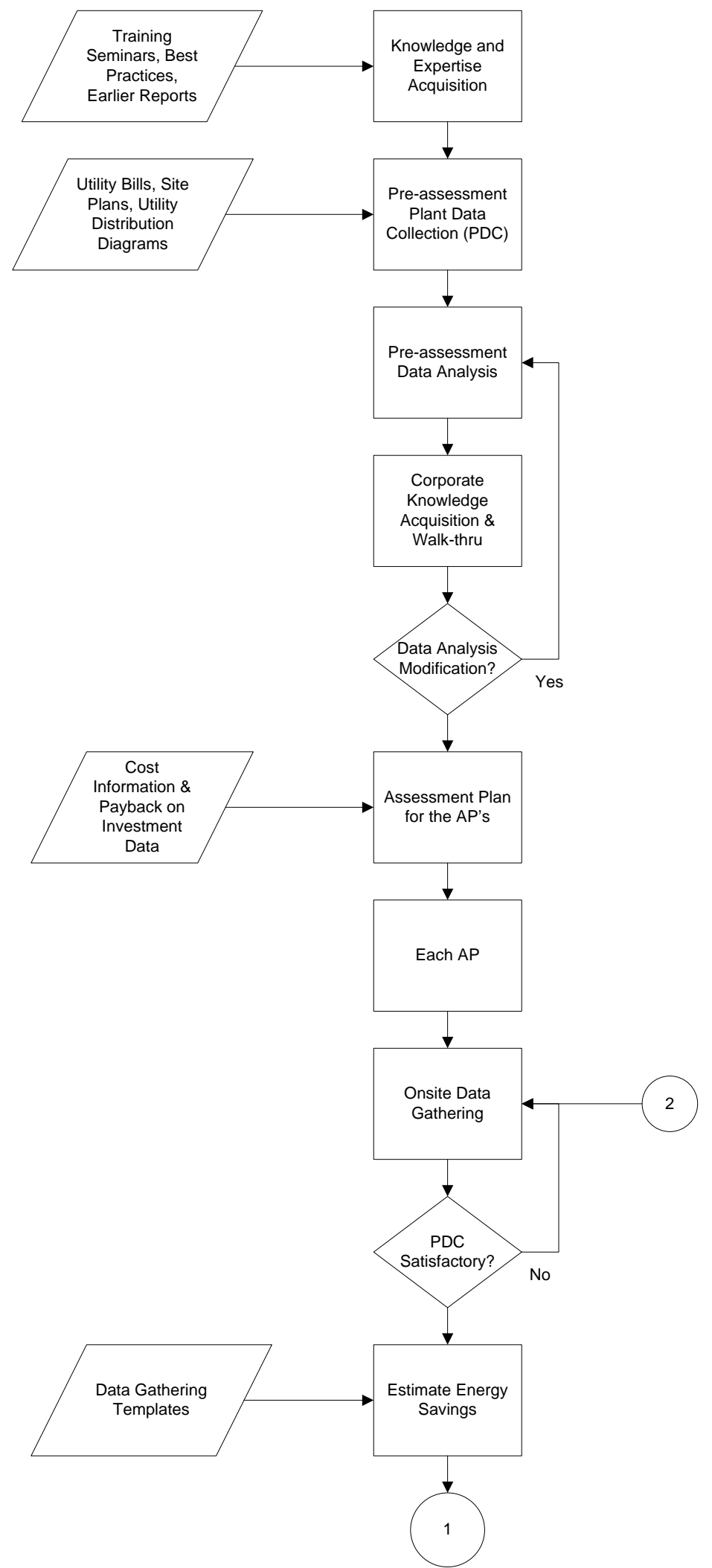

Figure 3.1: Methodology for Plant-wide Energy Assessments 


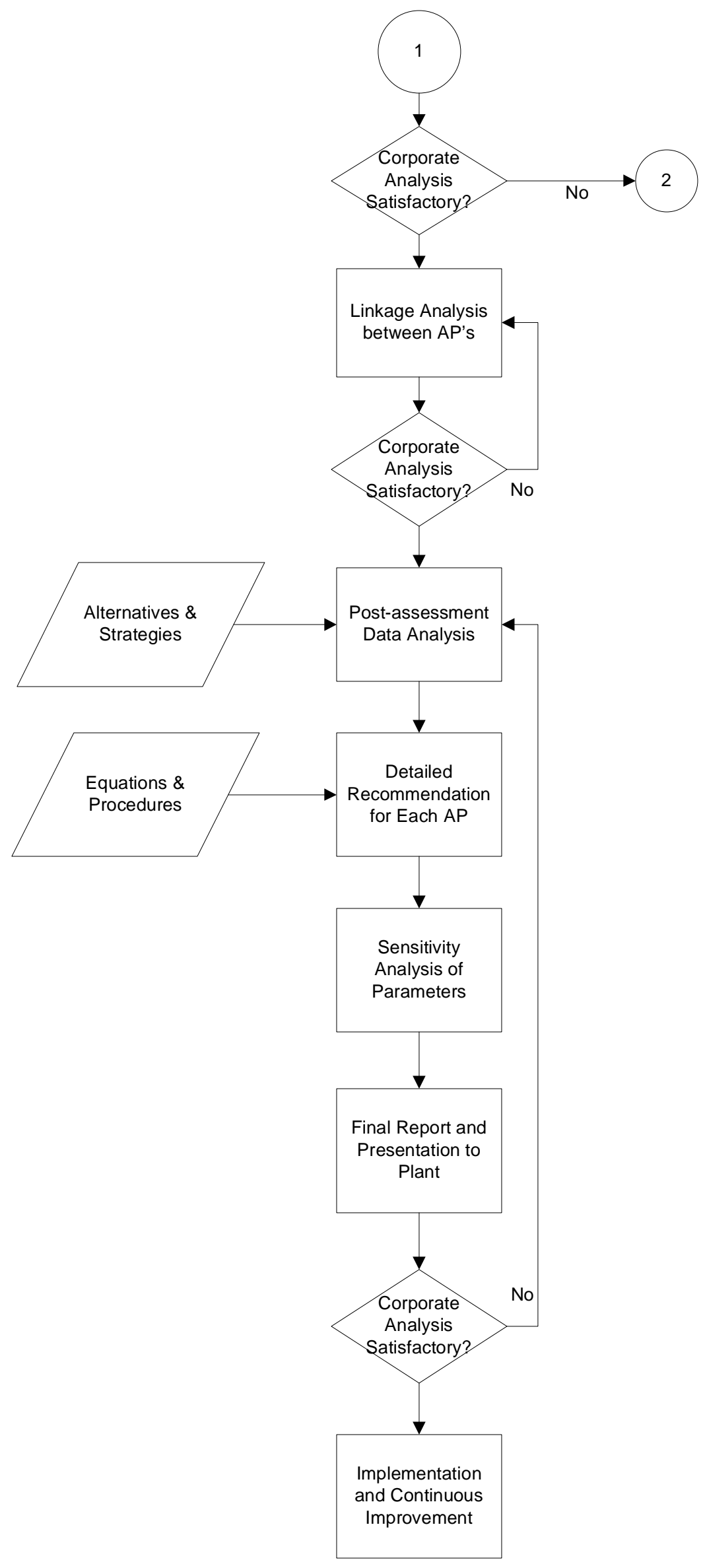

Figure 3.2: Methodology for Plant-wide Energy Assessments (continued) 
Energy assessments require complex and thorough research by knowledgeable and competent persons to provide practical and realistic results [7]. The figures (Figure 3.1 and 3.2) illustrate the flowchart for the methodology to perform a plant-wide energy assessment. The following methodology illustrates the major steps involved in performing a plant-wide energy assessment:

\section{Knowledge and expertise acquisition}

This step involves the process of gathering information on the procedures for performing energy assessments, developing equations and procedures for energy savings calculations. It involves analysis of the literature, utilization of the results obtained from previous assessment experience, and using information obtained from experts on the team.

\section{Pre-assessment Plant Data Collection (PDC)}

This step involves collecting information on the plant energy usage and its processes and it includes the following:

a. Process flow chart (PFC)

This chart gives the sequence of operations performed to convert the raw material into the final product desired.

b. Utility flow chart (UFC)

This chart gives the various sources of energy, like electricity, natural gas, fuel oil, etc., being used by each of the processes in the PFC.

c. Energy flow chart (EFC)

This chart shows the amount of energy being used in various forms like steam, hot and cold water, compressed air, etc., for each of the utilities in the UFC.

d. Throughput information from PFC (TPFC)

This chart provides information about the utilization of the various sources of energy from the UFC over a certain period of time. The usual single-day energy assessments start from this step. The data on the utilities usage is obtained for a one year period to gain an idea about the usage patterns and the rates involved. 
e. Future modifications to the PFC, UFC, TPFC

This illustrates the plans for any future changes in the plant over a period of time that might affect the PFC, UFC, or the TPFC. For example, there might be a plan to increase the plant production capacity in another six months, or there might be a shutdown of one compressor completely.

\section{Pre-assessment Data Analysis}

This step involves the development of ranked priorities (RP) for each of the assessment points (AP) that are identified on the combined TPFC - UFC. This can be done by the use of pie charts to illustrate the major users of energy. For each of the RP's, a criteria and metric has to be developed for assessment. For example, a criterion for accessing the compressors would be that the average intake air temperature should not be very much higher than the ambient outside air available. A metric for the same would be that if the temperature difference between the two is more than $20{ }^{\circ} \mathrm{F}$ then the possibility of using the outside air for the intake to the compressor would be much more economical.

\section{Corporate Knowledge Acquisition and Plant Walk-through}

This step involves acquiring of the knowledge that is accumulated with the experienced plant personnel. They will have a heuristic for each of the process settings. Based on trial-and-error method, they would have acquired some settings that will give the best efficiency for a particular process. A walk-through of the plant has to be performed to acquire a better understanding of the various processes. The walk-through has to be done with the guidance of some experienced plant personnel to identify each and every process and its significance.

After this step, the data analysis step should be checked for any modifications that might be necessary based on the discussion with plant personnel. If need be, the ranked priorities, and the criteria and metric for the RP's have to be changed.

\section{Assessment Plan for the AP's}

This step involves the development of an assessment plan for the AP's along with the detailed modifications that have been done to the criteria and the metrics. This can 
be illustrated in a Gantt chart for better understanding. This plots the various AP's along with the duration for performing each of them against time. Also, templates have to be developed for assessing each of the AP's.

\section{On-site Data Gathering}

This step involves the actual on-site data gathering for each of the AP's in the assessment plan. Also in this step, the PDC has to be verified for accuracy. If it is not accurate, new data has to be gathered in the plant to correct the required information.

\section{Estimate of Energy Savings}

Once the data gathering phase is completed, the templates developed during the assessment plan stage are used to give an idea of the energy savings that can be obtained. This step is performed on-site and the information is discussed with the plant personnel to verify the data accuracy. The plant personnel might direct the data collection to a proper location in the plant for that particular AP to obtain the best results.

\section{Linkage Analysis}

Once the estimation phase is completed, the data is analyzed to check for any linkages between the various AP's. For example, if the recommendation is to insulate all the uninsulated steam pipe surfaces in a particular area in the plant, the area becomes cold during winter months and a separate space heater needs to be installed to maintain comfort levels. Analysis has to be performed to understand the trade-off between the two options and arrive at the cost effective one. This plan has to be discussed with the plant personnel and the best option selected.

\section{Post-assessment Data Analysis}

This step involves the analysis of the data off-site to arrive at the detailed recommendations for each of the AP's. The intent of analysis is to evaluate the energy economics and the dollar economics to help the plant personnel understand, evaluate and implement the recommendation. The recommendation will give detailed 
information on the necessary changes to be made, the annual energy and cost savings obtained, and the implementation costs incurred for the same.

\section{Sensitivity Analysis}

This step analyses the sensitivity of the change in various key parameters to the energy and cost savings. The various parameters are tweaked to obtain the best efficiency for a given set of conditions.

\section{Final Report and Corporate Analysis}

The various recommendations are compiled into a final report and presented to the plant personnel. The necessary modifications are performed to the report as per the discussions with the plant personnel.

\section{Implementation and Continuous Improvement}

Once the final report is accepted by the plant personnel, the recommendations are implemented. The changes are monitored after implementation to check whether the operations are performed efficiently. Changes are made continuously to the various parameters for each AP to obtain optimal operating conditions.

\subsection{Difference between a One-day Assessment and a Plant-wide Energy Assessment}

The methodology for plant-wide energy assessments is different from the one-day energy assessments in the following ways:

1. In the PDC phase, only PFC and TPFC are done. The energy usage patterns of the individual operations are not studied. The PFC is made only during the on-site data gathering phase.

2. Pre-assessment data analysis is not performed. There is no ranked priority in the assessments for any particular area. All the areas are assessed without any prioritization and general heuristics are used to identify the recommendations. Criteria and metric used are based on prior assessment experience.

3. There is no assessment plan for any particular assessment point. The areas are accessed in the order they are visited. 
4. Once the data is collected on-site, it is not compared with the PDC to look for any modifications that might be necessary.

5. The various assessment points are not analyzed for any linkage. All the recommendations for the AP's are individual and each of them is developed to indicate savings if applied separately.

6. Cost and energy savings are not analyzed for any sensitivity to the changes in key parameters to arrive at optimal settings for any assessment point.

7. Once the final report is compiled it is not presented to the plant for corporate analysis. There is no continuous improvement process done after the report is presented to the plant.

The plant-wide energy assessment is in much more detail than a normal energy assessment in that it gives an in-depth analysis of the various users of energy and the various recommendations to get the optimal efficiency from each of the operations.

\subsection{Knowledge and Expertise Acquisition}

An energy assessment benefits from professional judgment and experience, like most other engineering activities. They can provide important information on the major areas of energy usage and can lead to energy savings simply by making people aware of how energy is being used in the plant. It serves the purpose of identifying where a plant facility uses energy and identifies energy conservation opportunities. The person who conducts the assessment should be familiar with the systems and operations of the type of plant that is being assessed. The assessor should have received training to perform the assessments and also the safety involved in them. Materials that are provided by the U.S. Department of Energy (DOE) should be used in the assessment process.

In the assessment that was conducted in the chemical plant, information was collected about each of the possible areas for energy conservation in a manufacturing facility. The various tools and methods for assessing each of these areas were studied. The final reports from prior assessments in the Industrial Assessment Center were studied to understand and get detailed information about the formulae and procedures for arriving at the final energy savings in each of the areas. This helps in developing templates for the data gathering step and also in the final reporting of the results to the company. The 
literature related to each of these opportunities was reviewed to validate all the procedures and formulae. The main areas that were looked at were HVAC (Heating, Ventilating and Air Conditioning), boiler and steam systems, compressors, motors and pumps, insulation and infiltration, lighting and utility analysis. Templates were prepared from each of the energy saving recommendations (ECO) in the various areas.

The plant wide energy assessments also make extensive use of the BestPractices that are available through DOE to identify opportunities for energy savings and process efficiency. The results and findings from each of the assessments are characterized and the savings are documented to form BestPractices that can be replicated in other facilities and other industries for multiplied savings. Some of the BestPractices in each of the major areas are:

- Compressed Air

o Determine the Cost of Compressed Air for Your Plant

o Eliminate Inappropriate Uses of Compressed Air

o Minimize Compressed Air Leaks

o Heat Recovery with Compressed Air Systems

o Pressure Drop \& Controlling System Pressure

- Motors

o Eliminate Voltage Unbalance

o Reduce Pumping Costs through Optimum Pipe Sizing

o Replace V-Belts with Cogged or Synchronous Belt Drives

o Motor Management System

- Process Heating

o Check Burner Air to Fuel Ratios

o Preheated Combustion Air

- Steam

o Steam Generation

- Determine the efficiency of the steam generation system

- Determine the usage of steam and the costs to generate this steam

- Optimize excess air in the boiler to increase efficiency

- Maintain clean fire-side and water-side boiler heat transfer surfaces 
- Optimize blowdown to reduce Total Dissolved Solids in the system

o Steam Distribution

- Properly select, size, and maintain steam traps

- Insulate all distribution system pipes, flanges, and valves

o Steam End Use

- Blowdown of non-condensables from condensing equipment

- Determine usage of steam per unit of product produced

o Steam Recovery

- Identify how much condensate is presently recovered and returned to the boiler system

- Ensure that the condensate return piping, flanges, and valves are properly insulated

Analysis of the data using BestPractices will help in identifying energy saving recommendations for each of the areas.

\subsection{Pre-assessment Plant Data Collection}

During the energy assessment, information has to be collected about every utility stream in the plant and its usage details. Detailed information has to be collected about the energy entering the site, energy generated on the site, energy distributed on the site, energy consumed on the site, energy leaving the site, waste energy collected, treated, discharged or re-circulated in the site, and cost data. The various energy systems in the plant include electricity, fuel oil, natural gas, LPG, coal, steam, compressed air, chilled water, hot water, and potable water supply. The various waste streams and waste energy include waste water, solid wastes like dry combustible (paper), wet combustible (garbage), dry noncombustible (coal ash), wet noncombustible (sewage sludge) and liquid combustible wastes (waste oil). The next step in this process is to obtain or prepare site plans which show the location of all the buildings and equipments in the plant along with the electricity transmission lines, steam distribution lines, water lines and natural gas distribution lines. This helps in establishing the detailed energy usage in the plant.

In our energy assessment of the chemical plant, information was collected regarding the various electrical distribution lines in the plant, along with the supply 
transmission lines to the facility. Information on the different cooling towers and their capacities, and the users that the cooling towers serve was collected. The historic details of the maximum capacity, peak usage and the available unused capacity was obtained for each of the utilities, namely, steam, electricity, natural gas, process water, potable water, fire water, plant air, and nitrogen. The annual monthly data on the fuel to steam conversion was obtained. The steam production details were collected, which provides information on the various boilers in the plant, their fuel and steam capacities, turbines, pressure reducing valves (PRV) and the age of the boilers. The historical and forecasted average hourly demand for steam was obtained.

The steam distribution diagram to the various parts of the plant was collected, which illustrates both the high pressure side and the low pressure side of the steam header. The boilerhouse flow diagram was collected, which shows the flow of steam and water, namely, feedwater, makeup water, condensate return and blowdown to the various equipments in the boilerhouse. The systems diagram of the utilities department was obtained for the site. It illustrates the various purchased utilities, the inputs to the various onsite utilities generation equipment and the generated utilities, the distribution systems for each of the utilities, waste generated, and the connection equipment to the different users. The plant air generation and distribution diagram was obtained, which gives information about the different compressors in the plant and the distribution of air throughout the plant to the various users. The plant water generation diagram illustrates the various pumps that take the water from the river and the well, the sand filters, electric feeds to the pumps and the pipeline to the users. The fire water distribution diagram was obtained and it gives information regarding the various pumps spread around the plant and the distribution of the piping network. The boiler water generation diagram was obtained that illustrates the water generation in the boilerhouses. The condensate return system diagram illustrates the return piping for the condensate back to the two boilerhouses. The typical cooling tower system diagram was obtained and information was collected regarding the various users for each of the cooling towers and the operating conditions of these towers. The potable water generation and natural gas distribution diagrams are collected. 
Detailed cost information of the natural gas supplied to the plant over a period of two years was collected. This aids in estimating the natural gas costs that need to be used for the energy savings calculations and also to look for any seasonal pattern in energy usage. The budget costs are collected for steam, electricity, cooling tower water, plant water and potable water, demineralized water, plant and instrument air, nitrogen, refrigerated cooling and natural gas for the utilities department. The detailed historic monthly cost data calculations for steam produced was obtained for a period of two years, along with the usage details of purchased fuel and produced steam. A list of all the large motors in the plant was obtained. Any motor above $50 \mathrm{HP}$ was considered to be large.

Information should be obtained from the plant regarding any previous energy studies that had been done. In our assessment, co-generation studies had been performed in the plant and the reasons why it had not been implemented was collected. The cost information that they use for their internal energy conservation studies was obtained. The templates of their steam cost from uninsulated piping over different periods of time, cost of steam leaks for different plume lengths, cost of steam condensate, operating cost of electric motors, cost of compressed air leaks, and cost of nitrogen leaks were collected. The results of the energy conservation study conducted in the utilities department were obtained, describing the various opportunities, investments and the proposed annual savings. Information was also obtained regarding any future modifications to the various equipments in the plant, as this would help in avoiding any redundancy of the data. They were planning to take one of the boilers and one of the compressors out of service within the next year. Hence, it was not considered in the energy conservation opportunities.

\subsection{Pre-assessment Data Analysis}

After the initial data collection process is complete, the next phase is to determine how and where the energy is being used in the plant. The energy usage data is analyzed to find out whether there are any seasonal trends in the energy usage in the plant, whether the historical energy usage is increasing or decreasing. This step helps in revealing certain patterns and trends in energy usage. The data collected in the PDC is analyzed to arrive at the various areas that use energy significantly over the others. Based on the analysis of the PDC, it was determined that the steam system, electrical system, plant air 
distribution system, nitrogen system, and the plant water system need to be focused upon for performing the energy assessment. The scale of the numbers involved in each of the areas justifies the need for focusing on the utility distribution systems for performing the plant-wide energy assessment.

\subsubsection{Steam System}

From the analysis of the data on the various boilers in the plant, the individual areas that need to be focused upon during the assessment were determined. The average age of the boilers in the plant exceeds 30 years, which implies a strong potential for energy loss due to inefficient or incomplete combustion. Hence, the analysis of the boiler efficiencies along with the analysis of the efficiency of economizers on each of the boilers was determined to be one of the assessment points. The other assessment point was to look at the potential for returning more condensate back to the boiler as only around 30 percent of it was currently being returned. This will help in increasing the efficiency of the boiler system, as more condensate returned back to the boiler will result in fuel savings, and water and chemical savings. The next assessment point in this area was to look for steam leaks from the old steam distribution lines that run along the entire length of the plant. The plant has a lot of steam traps and survey of these traps for failure was one of the assessment points. One of the key areas in the boiler steam system is insulation, lack of which can lead to direct loss of heat from the system. The next assessment point is to analyze the insulation in the different areas in the plant and also, make use of the insulation software for doing the economic thickness calculations.

\subsubsection{Electrical System}

The annual electricity costs in chemical plant are substantial and most of this is used for driving large motors. Matching of the various applications to the size of the motors can result in substantial energy savings and hence was determined to be one of the assessment points in the electrical system. Most of the motors in the plant are quite old and hence may not be energy efficient. One of the AP's was to use the MotorMaster Plus software available from the DOE to determine which of the motors could be replaced with energy-efficient newer motors. The plant has a large number of pumps and improvement in their efficiencies can lead to significant energy savings. The next AP was 
to use the Pumping System Assessment Tool (PSAT) available from the DOE for analyzing the pump efficiencies for optimality. The motors that operate on the boilers and cooling towers often operate at partial load conditions. The energy usage can be reduced by having adjustable speed drives (ASD) on these motors, which makes use of the ASD software. Hydrogen is the by-product of one of the processes in the plant and was being used in the boilers as a fuel. The possibility of using it in a fuel cell to generate power to offset the cost of imported electricity could be investigated. Another AP was to investigate the possibility of applying combined heat and power (CHP) in the plant as the thermal and electrical loads vary all through the year.

\subsubsection{Plant Air Distribution System}

The plant has four large centrifugal compressors that feed the entire plant's compressed air needs both process and instrumentation needs. The air system is very complex maze of piping with several tie-in points and the compressors range in age from 15 to 30 years. One of the potential areas for increasing the efficiency of the compressors was to reduce the air intake temperature and by making use of efficient lubricating materials. Excess air is being dumped during off-peak demand durations due to lack of storage capacity. Hence, another area for saving this wasted energy was to investigate the possibility of saving this excess air. About ninety percent of the energy used in a compressor is wasted as heat. This heat can be recovered and used in some process or other related applications. This is a potential area for increasing the overall system energy efficiency. Air leaks in the system are a significant wastage of energy from the system and can lead to artificial demand. A $1 / 4^{\text {th }}$ inch leak in a system operating at 100 -psig will result in a loss of $\$ 5,588$ annually for an energy cost of 5 cents per kWh. Ultrasonic leak detectors can be used to detect the presence of air leaks in the system, as the air emits an ultrasonic sound as it comes out of the leak and is very directional in nature. Since the plant air distribution system is quite huge, there is a large potential for reducing energy costs in this area. Another potential area for saving energy in the processes is to reduce the pressure at which the compressed air is generated. 


\subsubsection{Nitrogen System}

The nitrogen used in the plant is distributed along several thousand feet of the piping. Even a $1 / 4^{\text {th }}$ inch leak in the nitrogen line can cost up to $\$ 170,000$ annually. A potential area in the nitrogen system will be to focus on detecting the leaks suing ultrasonic or infrared detectors. Subcontractors hired by the company conducted an exhaustive and complete survey of all the gas leaks in the system in the plant.

\subsubsection{Plant Water System}

The plant uses thousands of gallons of process water per minute and any wasted water will be a loss of energy due to pumping, distribution and treatment costs. An investigation of reducing water loss in the system is a potential area for cost savings.

\subsubsection{Criteria and Metrics}

Once all the assessment points are identified, the criteria and metric for performing the data collection are developed. For insulation in boiler surfaces, a criterion is that if the surface temperature of the pipe is relatively higher than ambient, it needs to be insulated. The metric for the same is that if the surface temperature is higher than $120^{\circ} \mathrm{F}$, it needs insulation. A criterion for boiler system efficiency is that the efficiency increases for increase in intake air temperature. The metric is that for every $40^{\circ} \mathrm{F}$ increase in the intake air temperature, the boiler system efficiency increases by one percent. A criterion for motor sizing would be that large motors are potential areas for replacing with energy-efficient motors. A metric would be that any motor that is larger than $10 \mathrm{HP}$ could be investigated for replacing with energy-efficient motors. A criterion for reducing the line pressure in air compressors would be that a reduction in the pressure would result in lesser power consumption. A metric for the same would be that for every two psig reduction in the line pressure, the power consumption reduces by one percentage. Around ninety percent of the energy used in compressors is wasted as heat and the heat can be recovered with an efficiency of up to ninety percent. A criterion for assessing the same would be that greater the heat recovered more the energy saved. The metric for the same would be that for every hundred cfm of air produced at full load; the heat recovered can produce 50,000 Btu per hour of energy that can be used in process related applications. The results of this phase are then compiled to be discussed with the plant personnel 
during the site visit. The potential areas for assessment are listed for each of the areas for subsequent discussions with the plant personnel. For the steam system, the potential areas for assessment are:

- Efficiency of boilers

- Efficiency of economizer system

- Efficiency of waste heat recovery system

- Steam leakage

- Steam Trap Operation

- Heat Losses

- Utilization of low-pressure waste steam

- Water wastage reduction

The potential areas or assessment points identified for the electrical system are:

- Sizing of motors

- Pumping system assessment

- Wastage of energy in motor applications (ASD)

- Generation of power from a fuel cell using hydrogen

- Investigate the application of combined heat and power (CHP)

The potential assessment points for the plant air distribution system are:

- Increasing the efficiency of air compression

- Improving air storage capacity

- Improving heat recovery

- Air leakage reduction

- Reducing pressure

The potential assessment point in the nitrogen system was to survey for leaks and in the plant water system was to survey for wastage.

\subsection{Corporate Knowledge Acquisition}

The results of the previous step are discussed with the plant personnel to arrive at the final list of potential areas for the energy assessment. The plant personnel have significant information about each of the areas that they control. They have heuristics for each of the system efficiencies based on trial and error over several years. It greatly helps 
during the assessment to discuss with the plant personnel the progress in each of the areas in the assessment plan. It can lead to areas that had previously been overlooked and also to avoid errors in the onsite data collection and analysis processes. This step is performed during the kick-off meeting in the plant where the assessment team meets up with the plant personnel to discuss the assessment process. The main purpose of the kick-off meeting is to get the assessment team acquainted with the different plant maintenance personnel for each of the different assessment areas and to gather information from them regarding their individual areas. It also helps to prioritize the major areas in the assessment plan.

During the kick-off meeting in the assessment of the chemical plant, information was discussed regarding the various focus points in each of the assessment areas. For the estimation of the boiler efficiency, information was collected about any prior combustion test done on the boilers, the age of the boiler and the burners, and the instrument that would be used for the analysis. For estimating the efficiency of the economizer system, information was obtained about the type of economizer installed on each boiler and their age. When checking about the waste heat recovery system in the plant, it was found that only about thirty percent of the condensate is currently being returned back to the boiler. The rest of it was being sent down the drain. This was a high potential area for energy savings in terms of heat, chemicals and water costs. This leads to the analysis of steam traps in the plant for proper operation. It was learnt that most of the steam traps were accessible and hence, another area for energy savings was to analyze the steam traps for proper working. It was learnt from the plant personnel that it had been a long time since the last steam leak survey had been performed in the plant. This area leads to direct savings in energy in the boiler system, as the steam lost in a leak leads to increased demand for steam in the system. It was also found that the last insulation survey on the boiler system surfaces had been done years earlier. Hence, this was given the highest priority next to the survey of steam leaks. The main equipments that were proposed to be used for assessing the insulation in the boiler system were the infrared gun and the thermal imaging camera. The thermal camera can be used to survey large areas for hot spots, while the infrared gun can be used to look at smaller areas inside the boiler rooms. 
In the case of the electrical system in the plant, it was proposed to come up with a detailed list of all the motors and their drives to aid in efficient motor management using the MotorMaster software available from the DOE. This software helps to make economic decisions to rewind or replace a motor in the event of a failure. Also, it was proposed to study the sizing of the various motors in the plant utility section. It was proposed to use the Pumping System Assessment Tool (PSAT) software to analyze the pumps in the plant. It was learnt from the plant personnel that the cooling tower and boiler fans operate at varying loads. Hence, it was proposed to analyze the application of Adjustable Speed Drives (ASD) on the fan motors to obtain electrical savings. In the previous few years, there had been continuous increase in the cost of natural gas, which is a primary fuel in many of the boilers in the plant. Hence, one of the potential areas was to investigate the application of Combined Heat and Power (CHP) in the plant and to compare it with the generation of power from a fuel cell using hydrogen. It was also discussed as to how much of power needs to be generated to meet the demands.

In the area of plant air distribution, it was understood that the plant had four centrifugal compressors with three of them running and one as a backup. Two of the compressors had throttling up to around seventy percent and the others had no throttling. Centrifugal compressors have poor efficiency when they are operated at part-load conditions. This suggested the possibility of further investigation into the increase of the air compression system efficiency. The compressors operated as individual systems with separate controls and there was a possibility of integrating them into a compressor system package using electronic compressor controls and to do some load shifting. Based on discussions with the plant personnel, it was found that they had recently conducted an exhaustive study of the nitrogen gas leakage in the entire plant and they were also reducing the water wastage in the plant. The opportunity for reducing the air pressure and also to look for air leaks were dropped as the plant personnel were doing an excellent job of maintaining the air compressor system. It was found that the possibility of utilization of low-pressure waste steam was not a viable opportunity for energy savings in the plant. These modifications were put into the original assessment plan to get the final list of opportunities and develop the priorities for assessing them. 


\subsection{Plant Walk-through}

A walk-through of the plant should then be performed under the guidance of experienced plant personnel. This helps to identify the various processes in the plant to gain a better understanding of how the energy is being used in the plant in the various processes. It helps to uncover certain areas that might have been overlooked in the initial discussions and helps to correct any discrepancies in the earlier steps. The walk-through is useful as a prelude to conducting the energy survey in the plant. The walk-through helps the survey team to get acquainted with the plant and it helps in dividing up the areas for subsequent assessment. It also helps to determine the specific instrumentation that would be necessary to conduct the assessment in each of those areas.

\subsection{Data Analysis Modification}

Once the earlier steps are completed, any modifications that need to be done to the data analysis step have to be completed. This helps to enhance the accuracy of the assessment and also, to help avoid any wastage of time and resources. The modifications that need to be done would be arrived at after the discussions with the plant personnel and also from observations during the plant walkthrough.

During our assessment, the modification to the data analysis step was reflected in the changes to the assessment plan opportunities. The various opportunities were changed based on the experience with the plant. The opportunity for utilizing the waste lowpressure steam was dropped. The water wastage reduction was changed to condensate return back to the boiler, which had threefold savings in reducing the waste water, reducing the cost of fuel and chemical savings.

\subsection{Energy Assessment Plan}

In order to develop an assessment plant with the best possible assessment opportunities that would be feasible to assess in the plant, all the different opportunities must be analyzed in detail and none of them should be excluded because it seems impractical or is contrary to past practices. The current situation in the plant should be involved in the analysis as the base case, so that the other opportunities can be compared 
to it to verify whether it is a better opportunity and how much of improvement it brings to the existing situation.

While analyzing large plant, a large number of energy conservation opportunities might be generated and it may not be practical to analyze each and every one of them given the time and resource constraints [50]. This problem is overlooked by performing an incomplete analysis of the opportunities that are considered to be important by the assessment team and the plant personnel, and a small subset of the different opportunities is analyzed for feasibility. A more acceptable solution to overcome this problem would be to use a hierarchal approach to do the analysis of the various opportunities as indicated in the following flow diagram (Figure 3.3).

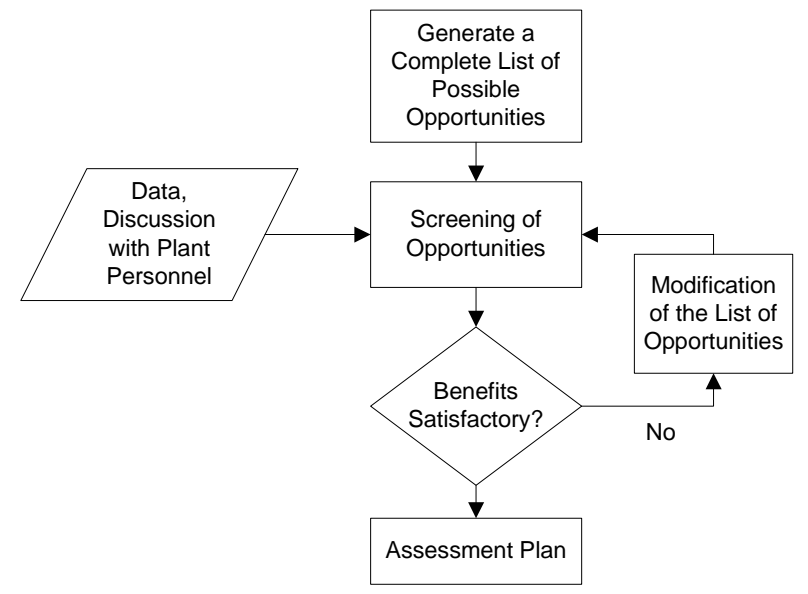

Figure 3.3: Screening of Energy Assessment Plan

The first step in this approach is to generate a list of all the possible energy conservation opportunities in the plant. These opportunities are then screened based on factors like the information from the plant personnel, their importance to the plant, the benefit/cost analysis of each of the opportunities, energy savings potential and their implementation potential along with their payback period, in order to check the feasibility and importance of the individual opportunities. This process generates a list of opportunities, which is a subset of the earlier list, that are promising, sufficiently feasible and beneficial to merit through the evaluation process in the assessment. The various opportunities are then ranked based on a weightage of the earlier factors considered. If there are two similar opportunities, the opportunity which gives the most savings is 
considered in the final assessment plan. The final list of opportunities for the energy assessment at the chemical plant was the following:

- Adjust Boiler air-fuel ratio

- Efficiency of Economizers

- Burner Replacement

- Steam Leaks

- Insulation

- $\quad$ Steam Traps

- Condensate recovery

- Motor Efficiency and Sizing

- Variable speed drives on the cooling tower pumps

- Pump Assessment

- Fuel Cells

- Investigate CHP: Sensitivity Analysis \& Literature Collection

- Efficiency of Air Compression

- Refrigeration

- Coal Gasification Power Generation Study

Of these opportunities, the insulation and steam leaks were given the highest priority, followed by the efficiency calculations for the boilers, burners and economizers. The plan is then plotted on a Gantt chart to illustrate the time limit for performing the individual assessments in each area. The Gantt chart in our assessment is as follows (Table 3.1) for a period of one year:

\begin{tabular}{|c|c|c|c|c|c|c|c|c|c|c|c|c|}
\hline Months & 1 & 2 & 3 & 4 & 5 & 6 & 7 & 8 & 9 & 10 & 11 & 12 \\
\hline Hold project kick-of & & & & & & & & & & & & \\
\hline Gather, analyze, an & & & & & & & & & & & & \\
\hline Develop compreher & & & & & & & & & & & & \\
\hline Identify best opport & & & & & & & & & & & & \\
\hline $\begin{array}{l}\text { Recommend follow } \\
\text { new technologies }\end{array}$ & & & & & & & & & & & & \\
\hline Prepare final report & & & & & & & & & & & & \\
\hline
\end{tabular}

Table 3.1: Project Milestone Chart for the Energy Assessment

Templates are then prepared for the assessment in each area. For the area of insulation, information is collected about the location of the lack of insulation, the number of similar uninsulated areas in that particular location, the diameter and length of 
each pipe, the surface area of exposed surface, the temperature of the surface, and the profile of the pipe, whether it is a vertical or horizontal pipe, vertical surface, or the top or bottom of a tank surface. A sample insulation template is shown in Table 3.2.

\begin{tabular}{|c|c|c|c|c|c|c|c|}
\hline No. & Quantity & Location & Diameter & Length & $\begin{array}{c}\text { Surface } \\
\text { area }\end{array}$ & Temp & Profile \\
\hline Units & & & in & $\mathrm{ft}$ & Sq.ft. & $\mathrm{F}$ & VP/HP/VS \\
\hline 1 & & & & & & & \\
\hline 2 & & & & & & & \\
\hline 3 & & & & & & & \\
\hline 4 & & & & & & & \\
\hline 5 & & & & & & & \\
\hline 6 & & & & & & & \\
\hline 7 & & & & & & & \\
\hline 8 & & & & & & & \\
\hline 9 & & & & & & & \\
\hline 10 & & & & & & & \\
\hline
\end{tabular}

Table 3.2: Data collection template for pipe and surface insulation

The template for assessing the area of steam leaks would require information on the location of the steam leak, the pressure and temperature of the steam that is flowing through the steam lines, and the plume length of the steam leak. The plume length is the length of the steam from the point of leak to the place where the leak just becomes invisible or the water condenses out of the leak. The assessment has to be performed with plant personnel since it requires the identification of the specific area of each leak, as they would have a proper name for identifying each area. This helps in proper implementation of the project once the reporting process is completed. The figure (Table 3.3) in the following page shows the template for assessing steam leaks.

Some of the information that would be required to assess the area of lighting in the plant are the location of each building or place that is lit, the type of lighting in the place like incandescent bulbs, fluorescent lamps, high intensity discharge (HID) lamps, etc., the light level measured in foot-candles (fc) by the light meter, the number of fixtures of the lamps and the number of bulbs in each lamp. There can be one more column to indicate the specific recommendation for each area like reduce lighting, occupancy sensors, switch from $400 \mathrm{~W}$ metal halide $(\mathrm{MH})$ to $360 \mathrm{~W} \mathrm{MH}$, etc. The template for this area is shown in Table 3.4. 


\begin{tabular}{|c|c|c|c|c|}
\hline No & Location & Pressure & Temp & Plume Length \\
\hline Units & & $\mathrm{psi}$ & $\mathrm{F}$ & Feet \\
\hline 1 & & & & \\
\hline 2 & & & & \\
\hline 3 & & & & \\
\hline 4 & & & & \\
\hline 5 & & & & \\
\hline 6 & & & & \\
\hline 7 & & & & \\
\hline 8 & & & & \\
\hline 9 & & & & \\
\hline 10 & & & & \\
\hline
\end{tabular}

Table 3.3: Template for assessing steam leaks

\begin{tabular}{|c|c|c|c|c|c|c|}
\hline No & Location & Type of lighting & $\begin{array}{c}\text { Light } \\
\text { level }\end{array}$ & $\begin{array}{c}\text { \# of } \\
\text { fixtures }\end{array}$ & $\begin{array}{c}\text { \# of bulbs } \\
\text { per fixture }\end{array}$ & Recommendation \\
\hline Units & & & fc & & & \\
\hline 1 & & & & & & \\
\hline 2 & & & & & & \\
\hline 3 & & & & & & \\
\hline 4 & & & & & & \\
\hline 5 & & & & & & \\
\hline 6 & & & & & & \\
\hline 7 & & & & & & \\
\hline 8 & & & & & & \\
\hline 9 & & & & & & \\
\hline 10 & & & & & & \\
\hline
\end{tabular}

Table 3.4: Template for assessing the lighting in the plant

The next template is used to collect detailed information about the various motors in the plant. The data collected in this template is used to compile a detailed list of motors in the plant, which helps in efficient motor management through the use of the MotorMaster software available through the DOE. The various data that would be required are a description of the motor, which illustrates its location and purpose, the number of similar motors in the area, the size of the motors in HP, load and usage factors, the annual operating hours of the motor, the rated speed from the nameplate of the motors in rpm, the measured speed from the measuring equipment in rpm, the number of poles that helps in determining the synchronous speed in rpm, and whether the drive has a v- 
belt or not. The following figure (Table 3.5) illustrates the template for the motor information collection.

\begin{tabular}{|c|c|c|c|c|c|c|c|c|c|}
\hline No & Motor Description & Number & Motor Size & $\begin{array}{l}\text { Load } \\
\text { factor }\end{array}$ & $\begin{array}{l}\text { Usage } \\
\text { factor }\end{array}$ & $\begin{array}{c}\text { Operating } \\
\text { hrs }\end{array}$ & $\begin{array}{l}\text { Rated } \\
\text { Speed }\end{array}$ & $\begin{array}{l}\text { Measured } \\
\text { Speed }\end{array}$ & V-Belt ? \\
\hline Units & & & $\mathrm{HP}$ & & & $\mathrm{Hrs} / \mathrm{Yr}$ & RPM & RPM & Yes/No \\
\hline 1 & & & & & & & & & \\
\hline 2 & & & & & & & & & \\
\hline 3 & & & & & & & & & \\
\hline 4 & & & & & & & & & \\
\hline 5 & & & & & & & & & \\
\hline 6 & & & & & & & & & \\
\hline 7 & & & & & & & & & \\
\hline 8 & & & & & & & & & \\
\hline 9 & & & & & & & & & \\
\hline 10 & & & & & & & & & \\
\hline
\end{tabular}

Table 3.5: Template for motor data collection

The template that is used for assessing the boilers would require data to be collected on the various fuels fired in the boiler, the flow rates of the different fuels to the burners in the boiler, the flow rate of the combustion air, the stack oxygen percent and the excess air percent from the stack analysis, the steam flow rate, the temperature and pressure conditions of the steam, the feedwater temperature, the flue gas temperature, the furnace dimensions, the fan data, and any other information from the boiler nameplate. The following figure (Table 3.6) illustrates the template for the boiler data collection.

\begin{tabular}{|l|l|}
\hline Fuel Used & \\
\hline Flow rates & \\
\hline \multicolumn{1}{|c|}{ Fuel } & \\
\hline \multicolumn{1}{|c|}{ Flue Gas } & \\
\hline \multicolumn{1}{|c|}{ Air } & \\
\hline Stack Oxygen percent & \\
\hline Excess air percent & \\
\hline Steam Rate & \\
\hline \multicolumn{1}{|c|}{ Temperature } & \\
\hline & \\
\hline Feedwater temp & \\
\hline Flue gas temp & \\
\hline Furnace dimensions & \\
\hline Fan data & \\
\hline Boiler Nameplate Details & \\
\hline
\end{tabular}

Table 3.6: Template for boiler data collection

The template for the data collection in the economizers on each boiler requires information about the type of economizer, the temperatures of the various fluids involved 
like the water inlet and outlet, and the flue gas inlet and outlet, and the water flow rate through the economizer. The following figure (Table 3.7) illustrates the template for the data collection for economizers.

\begin{tabular}{|l|l|}
\hline Water in temp & \\
\hline Water out temp & \\
\hline Gas in temp & \\
\hline Gas out temp & \\
\hline Type of economizer & \\
\hline Water flow rate & \\
\hline
\end{tabular}

\section{Table 3.7: Template for Economizer data collection}

In order to collect the data on the burners, the template requires the type of burner, the installation date or the age of the burner, specific fuel consumption of the burner, the firing rate of the burner and the nameplate details on the burner. The following figure (Table 3.8) illustrates the template for burner data collection.

\begin{tabular}{|l|l|}
\hline Date of installation & \\
\hline Type of burner & \\
\hline Fuel Consumption (sfc) & \\
\hline Firing Rate & \\
\hline Burner Nameplate Details & \\
\hline
\end{tabular}

Table 3.8: Burner data collection template

Once all the templates are developed for each of the assessment areas, the various instrumentation are used to collect the data for subsequent analysis, which is illustrated in the next step.

\subsection{Onsite Data Gathering}

Measurements using appropriate instrumentation are essential to the energy management process. The information that was obtained during the plant data collection should be verified by the audit team. It is convenient to use the data sheets that were prepared in the previous step and should represent the best information on the individual systems that can be obtained economically. If possible the data utilized for the analysis should cover twelve recent consecutive months. The operating hours of the various equipments should be determined as and when they are analyzed. This helps in determining the annual savings that can be obtained by implementing some conservation measure on the particular equipment. 


\subsubsection{Insulation}

The infrared gun and the thermal imager are used to measure the hot zones in the various areas in the plant. Infrared energy is an invisible part of the electromagnetic spectrum that exists naturally in objects and can be measured by heat-sensing equipment. The differences in the infrared emissions from the surface of the object that is being scanned causes various color codes to appear on the screen of the scanner, indicating different temperature zones. The hotter the object, more the infrared radiated from the object surface. The thermal imager should be used on a cloudy day in order to get the best possible thermal images for outdoor overhead lines. For best results, the thermal image should be matched with a digital image of the same location. This helps in positive identification of the area that needs the corrective actions and to better visualize the problem. During the initial visits to the plant, the insulation was given the highest priority. The templates were used to collect the data on the insulation lacks in various areas of the plant.

The infrared gun was used in easily accessible areas and the thermal imager was used to collect information on outdoor and indoor overhead lines. It has to be noted that wherever possible the infrared point should be focused on some black spot on the surface of the object, in order to get accurate temperature readings. Using it on shiny surfaces tends to reflect the infrared rays and does not give an accurate reading. When the gun is used to measure outdoor lines that are quite far, the suspended particles in the air tend to disperse the rays and not give an accurate value. The various plant surveys have to be done with accompanying plant personnel. This helps in positive identification of the exact location of the energy saving opportunity. Information had to be collected about the exact location of the insulation leak, a brief description of the object, the dimensions of the object, and also the temperature of the surface and the fluid inside. As a rule of thumb, any object with a surface temperature more than $120^{\circ} \mathrm{F}$ was selected for insulation study. Main areas for insulation leaks are steam valves, flanges and the end drums of the boilers among the other areas. Sometimes, the entire condensate return pipe line might be left uninsulated. A sample of the data collected for insulation is shown in Table 3.9. 


\begin{tabular}{|l|c|c|c|c|c|c|}
\hline \multicolumn{1}{|c|}{ Location } & Quantity & Diameter & $\begin{array}{c}\text { Equiv } \\
\text { Length }\end{array}$ & $\begin{array}{c}\text { Exposed } \\
\text { Area }\end{array}$ & Temp & Profile \\
\hline & & Inches & Inches & Sq. ft. & F & \\
\hline Steam Drum vent valve & 1 & 1 & 12 & - & 500 & V \\
\hline Relief Valve spool piece & 5 & 2 & 24 & - & 500 & V \\
\hline Steam Drum manway & 1 & 16 & - & 3 & 500 & VS \\
\hline $1 "$ valve on E of steam drum & 2 & 1 & 2 & - & 500 & H \\
\hline $1 / 2$ " valve on steam header & 1 & 0.5 & 1 & - & 500 & H \\
\hline $1 "$ valve on steam header & 1 & 1 & 2 & - & 500 & $\mathrm{H}$ \\
\hline HP steam export meter flanges & 1 & 8 & 16 & - & 620 & $\mathrm{H}$ \\
\hline 1 1" Super heater drain valves & 2 & 1 & 2 & - & 620 & V \\
\hline $10 "$ Mud drum ends & 4 & 10 & - & 16 & 500 & VS \\
\hline Blowdown valve \& set of flanges & 1 & 1 & 2 & - & 500 & H \\
\hline valve on steam export header & 1 & 1 & 2 & - & 620 & V \\
\hline $1 "$ valve on W of steam drum & 3 & 1 & 72 & - & 500 & H \\
\hline
\end{tabular}

Table 3.9: Sample of the data collected on Insulation

The profile indicates the orientation of the pipe or surface. ' $\mathrm{V}$ ' indicates that it is a vertical pipe, ' $\mathrm{H}$ ' indicates that is a horizontal pipe, and 'VS' indicates that it is a vertical surface. During the data collection for the insulation, information is collected on the insulation material and jacket materials used in the plant, the fuel prices that are used internally, the higher heating value of the fuel used, the annual hours of operation and the labor rate for maintenance personnel.

The medium or fluid inside the pipe or surface has an impact on the outside surface temperature and also decides whether the surface should be insulated or not. The temperature of the fluid that is transferring the heat has an effect on the amount of heat loss from the system. For instance, if we consider a steam pipe of diameter two inches that has one inch of insulation on it for two different temperatures; we find that the heat loss from the pipe surface is different in both cases for the same operating conditions. If the ambient temperature is $70^{\circ} \mathrm{F}$ and the wind velocity is zero, we find that for a two inch pipe operating at $350^{\circ} \mathrm{F}$ with one inch of insulation, the heat loss is $88.75 \mathrm{Btu} / \mathrm{hr} / \mathrm{ft}$ when using cellular glass pipe insulation with oxidized aluminum jacket material. If the inside fluid temperature is $500^{\circ} \mathrm{F}$, we find that the heat loss from the pipe surface is 160.5 
$\mathrm{Btu} / \mathrm{hr} / \mathrm{ft}$. The heat loss is nearly double for the different temperatures of the fluid in the pipe. The heat loss per unit of outside surface area for pipe insulation is given by:

$$
\frac{Q}{A}=\frac{\left(t_{p}-t_{s}\right)}{\left(R+R_{s}\right)}
$$

Where,

$$
\begin{aligned}
& \mathrm{Q}=\text { Heat loss from the surface, Btu/hr } \\
& \mathrm{A}=\text { Length of the pipe that lacks insulation, } \mathrm{ft} \\
& \mathrm{t}_{\mathrm{p}}=\text { Process temperature, }{ }^{\circ} \mathrm{F} \\
& \mathrm{t}_{\mathrm{s}}=\text { ambient temperature, }{ }^{\circ} \mathrm{F} \\
& \mathrm{R}=\text { Conduction resistance } \\
& \mathrm{R}_{\mathrm{s}}=\text { Surface resistance }
\end{aligned}
$$

From the equation, we find that the heat loss is directly proportional to the difference in the temperatures between the fluid inside the pipe and the ambient. Hence, the temperature of the fluid flow inside the pipe or surface has a direct effect on the need for proper insulation of the surface. In some cases the pipe may be left uninsulated to satisfy a specific purpose. For example, if the fluid inside has to be cooled before it reaches another location, the pipe is left uninsulated. These conditions have to be considered before suggesting insulation on any surface.

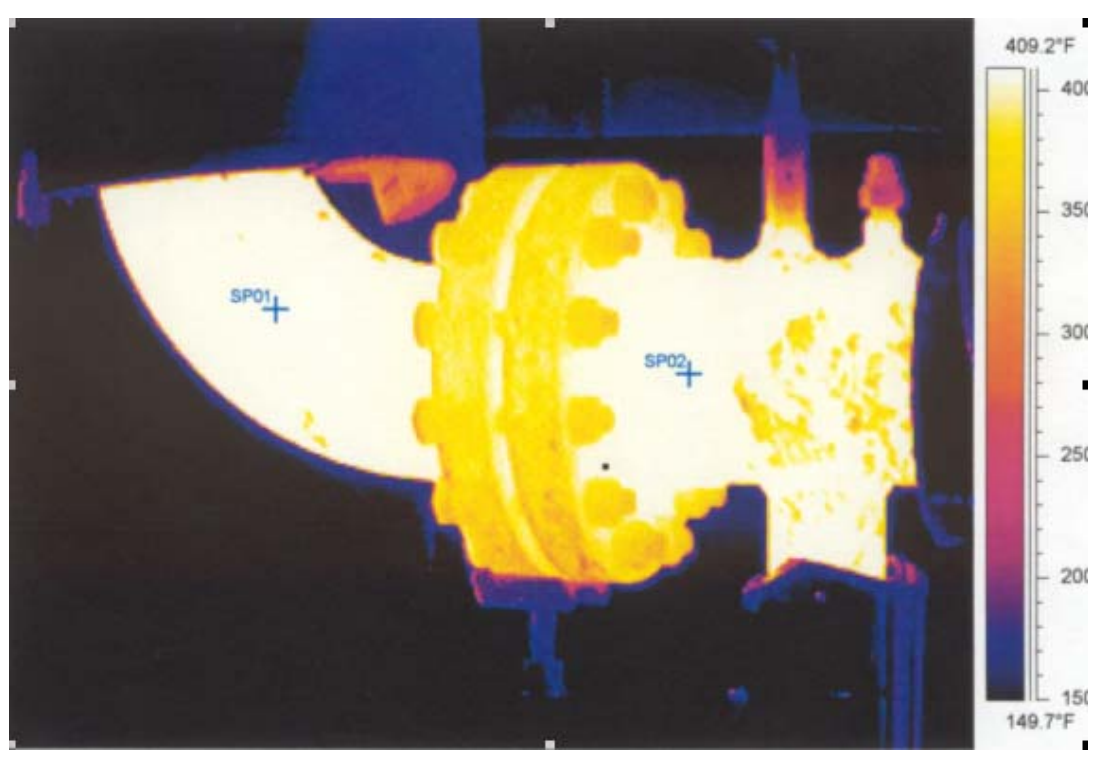

Figure 3.4: Thermal image of uninsulated pipe and flange 
The thermal image (Figure 3.4) shows a 12" diameter uninsulated line and a flange in the north end corner of boiler $\# 8$. Both the line and flange are at temperature of around $410^{\circ} \mathrm{F}$ and need to be insulated to prevent the loss of heat from the system. The thermal imaging helps in scanning large areas for uninsulated surfaces. The cold areas in the image are indicated by blue color and the hot areas are indicated by yellow color.

\subsection{2 $\underline{\text { Steam Leaks }}$}

With the help of plant personnel, the various steam lines around the plant are observed for any indication of leaks. The steam leaks are visually identified by the appearance of a white column of steam at the point of leakage. There are two methods that can be used for steam loss detection. They are the hole-size method and the plume length method. In the hole-size method, the steam losses are determined by estimating the diameter of the hole through which the steam leak occurs. This method can be applied to leaks that occur on ground surface. The diameter of the hole is approximated visually and the loss determined. The diameter cannot be measured exactly as the temperature at the point of the leak will be very high. The plume length method is usually applied when the steam leak is in an overhead line. The steam loss is calculated by estimating the length of the plume or the visual steam leak. The plume length is the distance from the start of leak (hole) to the point of the leak where the steam just becomes invisible. The estimated plume length is used for the calculation of the steam loss. Steam leaks contribute to direct heat loss in the steam distribution system. Steam will cause an increase in boiler load and makeup water consumption, as the steam lost has to be compensated by an addition of steam in the system to meet the needs. The loss associated with a steam leak is identical to that of vented steam. The most effective repairs are conducted when the system is out of service and depressurized. From the various visits to the plant, it was found that the steam leaks were in the overhead lines. All the steam is distributed to the various end users in the plant through overhead pipelines running across the entire plant. In order to detect the steam leaks, the entire plant has to be surveyed in detail as sometimes the leaks may be hidden in between lines. Based on discussions with the plant personnel, the location, temperature and pressure of the leaks were determined. The following table illustrates a sample of the data collected for steam leaks (Table 3.10). 


\begin{tabular}{|l|c|c|c|}
\hline \multirow{2}{*}{\multicolumn{1}{|c|}{ Location }} & Pressure & Temp & $\begin{array}{c}\text { Plume } \\
\text { Length }\end{array}$ \\
\cline { 2 - 4 } & psi & F & Feet \\
\hline C av. W of SPU station & 135 & 350 & 2.5 \\
\hline C av. W of SPU station & 135 & 350 & 2.5 \\
\hline C av. W of SPU station & 135 & 350 & 2.5 \\
\hline B av, W of 126 Cooling tower & 135 & 350 & 4 \\
\hline B av, W of 126 Cooling tower & 135 & 350 & 3 \\
\hline
\end{tabular}

Table 3.10: Sample steam leaks survey data

The following figure (Figure 3.5) shows the plume of a steam leak in an overhead line.

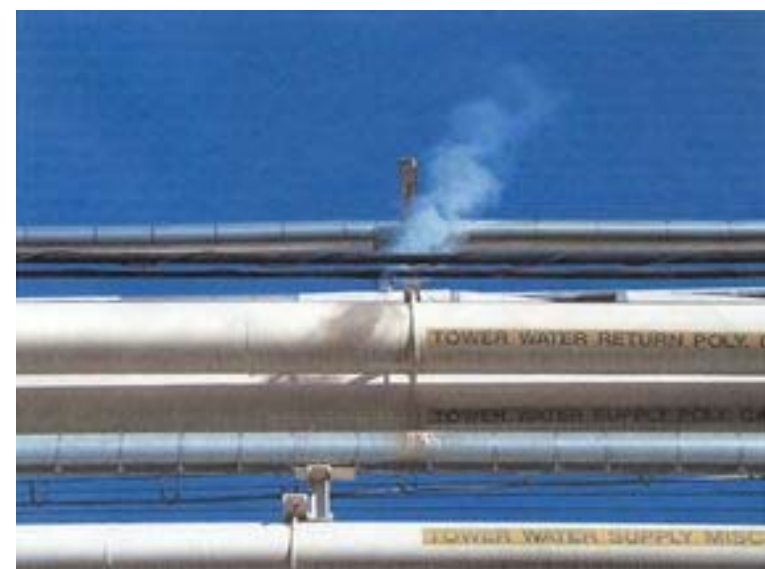

Figure 3.5: Steam Leak in an Overhead Line

\subsection{3 $\underline{\text { Steam Traps }}$}

Steam traps are automatic valves used in every steam system to remove condensate, air, and other non-condensable gases while preventing or minimizing the passing of steam. If condensate is allowed to collect in the system, it reduces the flow capacity of steam lines and the thermal capacity of the heat transfer equipment. If the air is not removed, it reduces the steam pressure and temperature, and acts as an insulator to impede heat transfer. If non-condensable gases are not removed, they can cause corrosion in the system. Finally, if steam is allowed to pass through the trap, it reduces the amount of steam in the system, thereby leading to increased production of steam to meet the demand. Radiation loss in the steam distribution lines causes water to form along the surface of the pipes. Steam traps remove this condensate as soon as they form which 
otherwise will reduce the heat transfer efficiency, reduce the steam quality, and cause water hammer in transportation lines.

All chemical plants have a large number of steam traps spread along the entire area of the plant. It will not be practical to analyze all the steam traps during the assessment. Hence a sample of traps is chosen for analysis using ultrasonic test equipment and also using visual examination. A steam trap working properly will open to release the condensate and flash steam, and close immediately in a cyclic process. The open-close cycle was observed in all the traps tested and the flash steam was observed in all of them. The ultrasonic kit was used to observe the sonic and ultrasonic sounds generated by the flow of steam and condensate, within the trap. The sounds were found to indicate normal operation of the steam trap. Hence, all the traps observed were working properly in our assessment.

\subsubsection{Burners}

The boiler houses were visited to get detailed information about each of the boilers in the plant. In each of the boilers, information was collected on the templates prepared and also any additional information about the specific boilers, namely some of the boilers might be firing a combination of fuels or they might be producing superheated steam unlike others. These specific conditions are noted so that they can be used during the analysis process. The detailed information collected for the boilers is useful for analysis of burners, economizers and the air-fuel ratio in the boilers.

\subsubsection{Variable Speed Drives}

A variable speed drive is an electronic device that controls motor speed by varying the frequency of the electrical supply. Most heating, cooling and ventilation systems operate at peak capacity even though demands are not at peak levels all of the time. For example, the amount of cooling energy required in commercial buildings and industrial processes vary throughout the year. The conventional response to changing demand for heating and cooling is to restrict flow to individual users, even while (peak) flow is maintained in the central system. This approach requires high motor energy and

reduces the lifetime of the equipment. A better approach is to use a variable-speed drive on cooling tower motors. These drives reduce air or water flow to meet changing loads. 
Variable-speed drives vary the frequency of AC electricity in response to an electrical signal. When coupled to a fan or pump motor, the change in frequency will result in a corresponding change in motor speed. Since the power required to drive centrifugal fans or pumps is proportional to the cube of the fan or pump speed, large reductions in electricity are achieved when fans or pumps operate at reduced speeds. In order to quantify the savings and also to justify the need for having a VSD on a pump, it is necessary to know how many hours (annually) the pump operates at a particular flow and it's loading during any point of time.

In our assessment, there were eight cooling tower systems operated by the utilities department that supply tower water to designated users. Cooling tower performance is weather dependent and restricts production in some areas during hot, humid weather. Several operating areas are supplied from the same tower. According to the discussions with the plant personnel, at a given time not more than 60 percent of the users connected to each cooling tower station are operating at the same time. Since the motor connected to the pump is constant speed, the cooling water to each user is kept running even if the user is not operating. This creates an opportunity for installing variable speed drive (VSD) on

the cooling water supply pump. Since peak conditions typically occur infrequently, substantial energy savings are possible by varying fluid flow rate to match the actual demand. In addition to substantial energy savings, other advantages of VSD's include smoother control, softer starts, reduced noise, reduced damper/valve maintenance, reduced flow friction heating, and faster response. Information that was collected during the data gathering process is the load factor on each of the pump motors, along with their rated power, motor efficiency and percent of the users that would be connected at any time.

\subsubsection{Compressed Air System}

Dynamic compressors are high-speed rotating machines in which air is compressed by the action of rotating impellers or blades that impart velocity and pressure to the air. This type of compressor will deliver air at an essentially constant pressure over a wide range of capacities. The direction of airflow is radial with respect to the axis of rotation. Centrifugal compressors have a lower limit of stable operation called the surge 
point. Operation below this point results in pumping or surging of the airflow. The use of centrifugal compressors is usually limited to high volume industrial plant applications, such as chemical manufacturing, textile plants, petroleum refining, or general plant air systems in large manufacturing facilities. The compressor operates at high speeds and therefore uses smaller, more compact equipment. Three stage centrifugal compressors are generally more efficient than rotary screw compressors and can approach the efficiency levels of double acting reciprocating compressors. Centrifugal compressors are best suited to applications where demand is relatively constant or in industrial plants where they can be used primarily for base-load operation, allowing other compressor types to be used as trim machines to meet peak demands.

In our assessment, there were four main centrifugal compressors with operating capacities of 2,500, 2,500, 2,500 and 3,000 scfm respectively. These compressors feed the entire plant's compressed air system for instrumentation and process needs. The compressors range in age from 13 to 30 years. Centralized monitoring of these units and metering of usages were not available. Also AC \#3 and AC \#4 have an inlet-throttling device, which have a throttling capacity of $70 \%$. There is no throttling available for AC $\# 1$ and AC \#2. As per the discussions with the utility department personnel, the plant instrumentation and process requires on an average a constant supply of $6600 \mathrm{scfm}$ of compressed air supply in the plant. Also it was noted that one of the four air compressors is always used as a stand by, so the remaining 3 units fulfill the demand of compressed air. Also it came up in the discussions that the AC \# 3 would be shortly taken out of service due to the probable close down of some units in the plant. Hence all our efforts in optimizing the compressed air system were centered on AC \#1, AC \#2 and AC \#4 in the plant.

The utilities department with their sophisticated electrical measuring equipment and constant perseverance, collected exhaustive data on the compressors AC \#1, AC \#2 and AC \#4. Continuous data like instantaneous power, demand, current, voltage, power factor, load duration curves, etc. was obtained from the measurements. This data was collected over two complete shifts in the plant. A brief summary of some of the collected data can be seen in the Table 3.11. 


\begin{tabular}{|c|c|c|c|c|c|c|c|}
\hline Compressor & $\begin{array}{c}\text { Recording } \\
\text { Duration }\end{array}$ & \multicolumn{2}{|c|}{$\begin{array}{c}\text { Instantaneous } \\
\text { Power (kW) }\end{array}$} & $\begin{array}{c}\text { Avg. } \\
\text { Power }\end{array}$ & \multicolumn{2}{c|}{$\begin{array}{c}\text { Total } \\
\text { Current (Amps) }\end{array}$} & $\begin{array}{c}\text { Load } \\
\text { Factor (\%) }\end{array}$ \\
\hline & & Min & Max & & Min & Max & Avg. \\
\hline \multirow{2}{*}{ AC \# 1 (centrifugal) } & $01 / 30$ to 01/31 & 457.30 & 499.00 & 478.15 & 215.8 & 234.2 & 95 \\
\cline { 2 - 8 } & $01 / 31$ to 02/01 & 460.10 & 508.10 & 484.10 & 217.2 & 238.5 & 95 \\
\cline { 3 - 8 } & $01 / 30$ to 01/31 & 464.50 & 619.50 & 542.00 & 219.2 & 294.3 & 80 \\
\hline AC \# 4 (centrifugal) & $01 / 31$ to 02/01 & 462.30 & 635.70 & 549.00 & 226.0 & 289.0 & 80 \\
\hline & $01 / 3$ &
\end{tabular}

Table 3.11: Brief summary of data collected on \# 1 and \# 4 AC

\subsubsection{Compressed Gas Leaks}

North American industry looses millions of dollars annually to undetected air, gas and steam leaks that rob efficiency during manufacturing and processing. Leaks can be a significant source of wasted energy in an industrial compressed air system, sometimes wasting 20-30 \% of a compressor's output. A typical plant that has not been well maintained will likely have a leak rate equal to $20 \%$ of total compressed air production capacity. The best way to detect leaks is to use an ultrasonic acoustic detector, which can recognize the high frequency hissing sounds associated with air and nitrogen leaks. These portable units consist of directional microphones, amplifiers, and audio filters, and usually have visual indicators or earphones to detect leaks. Leaks occur most often at joints and connections. Equipment no longer in use should be isolated with a valve in the distribution system. An exhaustive and complete survey of all the gas leaks in the system in the plant was conducted during the assessment by subcontractors hired by the company. This survey included air leaks, natural gas leaks and nitrogen leaks in the system. The results of the survey were quantified for each type of leak and also the location of the leaks was tagged. A sample of the data collected is shown in the following table (Table 3.12).

\begin{tabular}{|c|c|c|c|l|}
\hline Tag no. & System & CFM & 1000 CF/Year & DESCRIPTION and / or APPROXIMATE LOCATION. \\
\hline A5 & Air & 0.4 & 210 & Shut off to \#12 CT near breaker room on 11th St \\
\hline A6 & Air & 2.6 & 1,367 & Regulator south of \#12 CT on 11th St. \\
\hline 336 & N. Gas & 0.6 & 315 & Fitting for temperature gage by lower platform at IO meters. \\
\hline 344 & Nitrogen & 1.4 & 736 & Valve bonnet by lower platform at IO meters. \\
\hline 600 & Nitrogen & 1.4 & 736 & Pipe clamp leak on highline on north side of MR2. \\
\hline
\end{tabular}

Table 3.12: Air and Gas survey sample 


\subsubsection{Motor Management System}

A motor management system is designed to assist a facility to reduce energy costs by maximizing production efficiency, minimizing energy consumption, correcting for power factor, giving better understanding of the utility billing statements, and establishing a preventative and predictive maintenance program. Motor Master is capable of analyzing the energy and cost impacts of various hypothetical situations that occur either before and/or after a motor failure. These situations include downsizing to a smaller motor, rewinding a failed motor, replacing a failed motor with same size motor, and replacing a failed motor with a larger spare motor.

During the data gathering process, a detailed inventory of all the large motors (above $25 \mathrm{hp}$ ) was obtained during the plant visits. A motor load test was performed on all the large motors so as to investigate the possibility of downsizing. This test should be done over a shift or longer time period so that the exact load conditions imposed by the process on the motor can be examined. Using a power meter the electric power going into the motor is determined and compared with the motor's nameplate output power. An accurate power meter is necessary for load testing, rather than a simple ammeter because current is not a valid measurement if motor load below $60 \%$ of full load, due to large portion of the reactive power. The wattmeter must be true RMS, which prevents inaccuracies due to variations in power quality. Motor efficiency values can be obtained from the motor nameplate, manufacturer's data, or Motor Master software. The operating speed of the motor is measured by a tachometer and is used for calculating the load using the slip method.

\subsubsection{Flow Meter Sensitivity}

The flow rate or quantity of a moving fluid or gas is measured with the help of flow meters. It is critical to accurately measure the flow of a fluid in many industrial plants as it can make the difference between operating at a proper efficiency and at a poor efficiency. In some cases, inaccurate measurements can lead to disastrous results in the equipment that is being operated. The following figure (Figure 3.6) shows a mass flow meter for gases [46]. 


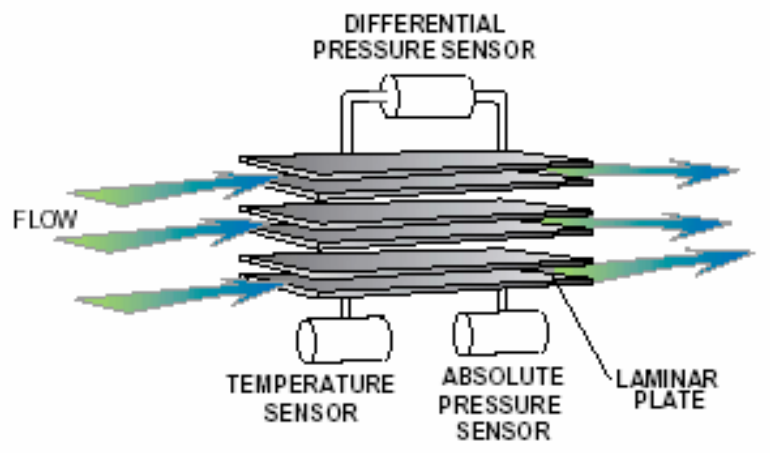

Figure 3.6: Working of a Flow Meter

The gas or fluid passes through the laminar flow element in the flow meter and the pressure difference is measured by the sensor, which is linearly proportional to the flow rate of the gas. The important specifications of a flow meter are the measuring range, type of the fluid, units of measurement, and operating pressure and temperature ranges. The coefficient of discharge can change and hence affect the accuracy of the meter if the internal surface develops a film of dirt, corrosion or organic growth [47]. Also if the pressure downstream of the meter is too low, cavitation can occur within the meter at high flowrates affecting its accuracy.

In our assessment at the chemical plant, it was observed that the flow meter for the natural gas to the burners in each of the boilers was not working properly. The flow of natural gas is a critical factor in calculating the efficiency of the boiler. Inaccurate flow measurements will fool the operator into thinking that the boiler efficiency is better or worse than it really is. The calculation of the efficiency of the boiler $(\% \mathrm{E})$ involves the gas flow to the burner as evident from the following formula [48]:

$$
\% E=\frac{S F\left(h_{1}-h_{2}\right)+B F\left(h_{3}-h_{2}\right)}{G F \times H H V}
$$

Where,

$\mathrm{SF}=$ Steam flow, $\mathrm{lb}$

$\mathrm{h}_{1} \quad=$ Steam enthalpy at the boiler operating pressure, Btu/lb

$\mathrm{h}_{2}=$ Feedwater enthalpy at the feedwater temperature, Btu/lb

$\mathrm{h}_{3}=$ Blowdown enthalpy at boiler operating pressure, Btu/lb

$\mathrm{BF}=$ Blowdown flow, $\mathrm{lb}$ 
$\mathrm{GF}=$ Gas flow, $\mathrm{cf}$

$\mathrm{HHV}=$ Gas higher heating value, Btu/cf

From the equation, we find that the efficiency of the boiler by the input-output method is inversely proportional to the flow of natural gas to the burner. Hence, any false reading given by the flow meter can adversely affect the value for the boiler efficiency. It will appear that the boiler is working at a better efficiency whereas it actually is working at a much lower efficiency. Hence, the meters should be calibrated every once in a while to ensure proper operation on the boiler.

\subsection{Estimate of Energy Savings}

In order to verify the accuracy of the data collected, an estimate of the possible energy savings is calculated after the completion of data gathering for each of the assessment areas. Hence, during the course of the data gathering process, once an area is completed, an estimate is made for the energy savings in that particular area and discussed with the plant personnel. This process will help the plant personnel to facilitate the completeness and accuracy of the data collected. And also this step helps in finding out whether the area requires more work to be done based on the magnitude of the anticipated savings.

In our assessment at the chemical plant, most of the efforts were prioritized for insulation as mentioned before. Initially data was collected on the templates for the data required for insulation and the potential energy savings were calculated and verified with the plant personnel. They gave a different heating value and natural gas rate that had changed over the time of performing the assessment. The corporate rate used internally in the company had changed and also, they pointed out certain areas that would be taken out of service within a certain period of time. The savings calculations were compared with the internal survey that they had done based on the areas mentioned in our data gathering process.

In order to quantify the savings from increasing the condensate recovery back to the boiler, it was found from the personnel that fifty percent of the steam is available as

condensate after performing the useful work and the cost of water, its disposal, the cost of 
chemicals, and the temperatures of the makeup water and the condensate were found. These data help in calculating the anticipated dollar savings in returning more condensate back to the boiler. Also discussions brought up suggestions that could induce the other areas of the plant to send the condensate back to the boiler. They suggested that if there were an incentive for the returned condensate, more departments in the plant would return the condensate rather than send it down the drain.

In the case of calculating the efficiency of the boilers, the data was collected on each of the boilers on the templates. Based on discussions with the plant personnel, it was

found that the meters for the fuel flow and the steam flow were quite old and not very accurate. This is necessary information that is needed to decide which method has to be used in order to arrive at the efficiency of the boilers. The heat loss method is the most accurate method for the calculation of the boiler efficiency, but it requires the knowledge of all the heat losses involved. And also, it requires the mass flow rate of fuel, which can be obtained only from the meters thereby trusting the accuracy of them. This method will involve a lot of assumptions in the process of estimating the various losses. Hence, we could directly use the input - output method. The input - output method requires the heat in the steam and the heat input to the boiler. This is calculated from the mass flow rate of the steam and the fuel that are obtained from the meters. This again goes back to trusting the meters. Using the combustion efficiency tables to calculate the efficiency gets around this problem.

\subsection{Linkage Analysis}

Once the data gathering and the savings estimation are done, the different assessment areas need to be analyzed to check for any linkage between different areas. This practice is often referred to as taking a "systems approach" because the focus is shifted from components to total system performance. The assessment areas are analyzed to find out how they affect the system as a whole rather than as individual components. Changes that are made in one assessment area might have an effect on the performance of another assessment area. The changes should be recommended only after complete satisfaction of the plant personnel with this alternative method. 
In our assessment, for the recommendation on replacing the burner on a boiler with a more efficient one, it reflects in not only improving the efficiency of the fuel burning process, but also in improving the overall boiler system efficiency. Based on discussions with the burner manufactures, it was found that replacing the old burner with a new one would increase the efficiency of the boiler by one to two percent by improving the combustion process. Also, in the case of installing the adjustable speed drives on the cooling tower pumps it was verified that this drive does not adversely affect the performance of the users connected to each of these towers. Inspecting and repairing the steam traps would affect the volume of condensate being returned back to the boiler. In our assessment all of the steam traps were operating properly and hence, this was not looked into in detail. Plugging the leaks in the compressed air system will increase the pressure in the lines a little and hence, care should be taken that the sensitive users are not affected.

\subsection{Post-Assessment Data Analysis}

Once the linkage analysis process is completed and the plant personnel are satisfied with the opportunities in the different assessment areas, the detailed recommendation should be developed. The data gathered in the prior phases is analyzed in detail to arrive at the changes that need to be done to the existing conditions in each of the assessment areas that would yield energy savings. Different alternatives and strategies are considered to come up with the best one that gives good energy savings. Various manufacturers of improved technologies for each area are consulted by the team to get the best possible savings in energy. The analysis involves computing the detailed energy and cost savings, the cost for implementation and the payback on the investment for each of the recommendations. This step helps in putting together a final report that would be provided to the plant personnel for them to understand, evaluate and implement the recommendations.

\subsubsection{Insulation}

In our plant-wide assessment, for the data analysis in the area of insulation, both the ASHRAE method and the $3 E$ Plus ${ }^{\circledR}$ software from North American Insulation Manufacturers Association (NAIMA) were used. The software provides ease of use and 
can be easily understood to get the calculations done quickly. This program is designed to provide a simplified method to model heat flow in insulated piping and equipment. The program can handle hot or cold surfaces, piping or flat surfaces. Figure 3.7 shows the main window in the NAIMA 3E Plus software.

During the various visits to the plant and discussions with the plant personnel, it was observed that the insulation was missing in many places. In many cases, the insulation had been removed to facilitate some repair work and after the repair work was completed, the insulation was not put back. In the remaining areas of the plant though, the insulation was well maintained. For the given conditions of temperature, geometry of the uninsulated area, and the operating conditions, the software gives the economic insulation thickness, the heat loss after the insulation is applied, the cost savings, and the surface temperature after insulation. From this we can obtain the energy savings by taking the difference between the heat losses from a bare pipe obtained from the software and the heat loss after insulation. The cost of insulation is given by the software for the given price of insulation for the particular operating conditions. The annual savings is the sum of the fuel saved, the cost of insulation and the cost of labor involved to fix the insulation. The pipes and surfaces can be insulated by installing strips of insulation. The same set of calculations can be performed using ASHRAE handbook of fundamentals, but it takes a longer time to do it for individual surfaces, particularly when there are large volumes of data to be analyzed.

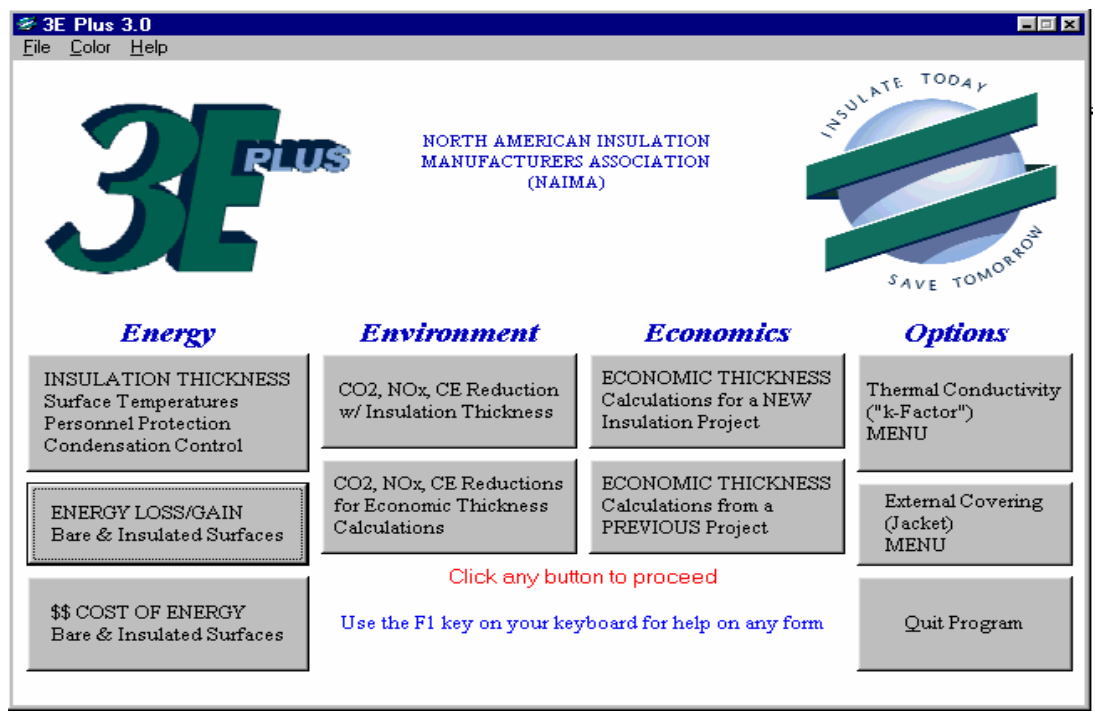

Figure 3.7: Main screen of the 3E Plus software 


\subsubsection{Steam Leaks}

In the case of steam leaks from overhead lines, the plume length method was used as explained earlier. The following Table illustrates the magnitude of the steam loss in lb/hr (Table 3.13).

\begin{tabular}{|c|c|c|c|}
\hline \multicolumn{5}{|c|}{ Steam loss - lb/hr } \\
\hline Plume Length & $45^{\circ} \mathrm{F}$ Ambient & $70^{\circ} \mathrm{F}$ Ambient & $90^{\circ} \mathrm{F}$ Ambient \\
\hline \multicolumn{5}{|c|}{$\mathbf{1 1 5}$ psi } \\
\hline $3 \mathrm{ft}$. & 10 & 30 & 50 \\
\hline $6 \mathrm{ft}$. & 30 & 170 & 280 \\
\hline $9 \mathrm{ft}$. & 70 & 420 & 700 \\
\hline $12 \mathrm{ft}$. & 110 & 650 & 1,100 \\
\hline \multicolumn{7}{|c|}{$\mathbf{4 1 5}$ psi } \\
\hline $6 \mathrm{ft}$. & 20 & 35 & 50 \\
\hline $9 \mathrm{ft}$. & 50 & 170 & 290 \\
\hline $12 \mathrm{ft}$. & 130 & 500 & 1,400 \\
\hline
\end{tabular}

Table 3.13: Plume Length Method

The data collected in the field visit in the plant were analyzed using Excel $^{\mathrm{TM}}$ spreadsheet. A typical steam unit cost was used. Table 3.13 was used for finding the steam loss (lb/hr). The plant annual operational hours are 8,760 hrs/yr. The steam loss is multiplied by $8,760 \mathrm{hr} / \mathrm{yr}$ to get steam loss in $\mathrm{lb} / \mathrm{yr}$. This is multiplied by the steam unit cost and divided by 1,000 to obtain the steam loss in $\$ / y r$. The total steam loss is calculated to be $\$ 35,070 / y r$ from the different steam leaks observed and is a savings if the leaks are plugged. Taking the regional labor rate of $\$ 40 / \mathrm{hr}$ and assuming that it takes 1 hour to repair a leak and a part cost of $\$ 30$ per leak [23], the total cost incurred is $\$ 1,330$ and hence, the payback is in one month. The reduction in the steam loss due to leaks also returns in energy savings in the boiler, which is calculated as follows:

$$
\mathrm{ES}=\left[\mathrm{SS} \times\left(\mathrm{h}_{\mathrm{s}}-\mathrm{h}_{\mathrm{w}}\right)\right] / 1,000,000
$$

Where,

$$
\begin{array}{ll}
\mathrm{ES} & =\text { Energy savings, MMBtu/yr [9] } \\
\mathrm{SS} & =\text { Steam savings, lb/yr } \\
\mathrm{h}_{\mathrm{s}} & =\text { Enthalpy of Steam at } 650 \mathrm{psig}, 1202.3 \mathrm{Btu} / \mathrm{lb}[20] \\
\mathrm{h}_{\mathrm{w}} & =\text { Enthalpy of water at } 203^{\circ} \mathrm{F}, 171.105 \mathrm{Btu} / \mathrm{lb}
\end{array}
$$


It is found that an energy saving of 5,513 MMBtu is obtained annually, which reduces the $\mathrm{CO}_{2}$ emissions by 622,969 lb/yr, as one MMBtu saved reduces the $\mathrm{CO}_{2}$ emissions by 113 lbs. The following actions are recommended to minimize steam leaks [7]:

A Conduct periodic surveys on the steam lines for leaks using appropriate acoustic and temperature probes or by visual inspection. Many important steam leaks are hidden, such as:

- Leaks in traps or valves discharging to sewer or condensate systems

- Leaking valves leading to idle equipment

- Leaks in heater or other equipment connected to the steam system

ג Shut off steam to equipment whenever is taken out of service

ᄉ Re-route piping, where practical, so that leaks are visible, rather than be hidden.

\subsubsection{Burner Replacement}

During the visits to the boiler houses, the operating conditions of the burners were noted. All the burners were supplied along with the boilers by the manufacturers in the early sixties and seventies, and hence are quite old. The burners on three of the boilers were retrofitted in the eighties. Replacing the burners with new ones can increase the efficiency because of the improvements in the burner design over the years. Talking to various vendors, if the old burner is replaced with a new one for the given operating conditions, there will be an improvement in the boiler efficiency of around two percent, since the burner on the boiler is quite old. The cost of a burner rated at $160 \mathrm{MMBtu} / \mathrm{hr}$ will be $\$ 40,000-\$ 50,000$ and the installation will be around $\$ 20,000$. Hence, taking equipment cost to be $\$ 45,000$ and the installation cost to be $\$ 20,000$, we get the total investment in replacing the burners to be $\$ 65,000$. The equations used to compute the savings obtained in replacing the old burners are:

$$
\begin{array}{ll}
\mathrm{U} & =\mathrm{FR} \times \mathrm{HHV} \times \mathrm{OH} \\
\mathrm{ES} & =\mathrm{U} *[1-(\mathrm{EFFC} / \mathrm{EFFp})]
\end{array}
$$

Where,

$\mathrm{U} \quad=$ Current annual gas energy usage by boiler, $\mathrm{MMBtu} / \mathrm{yr}$

ES = Energy savings, $\mathrm{MMBtu} / \mathrm{yr}$ 
$\mathrm{CS}=$ Cost savings, $\$ / \mathrm{yr}$

$\mathrm{FR} \quad=$ Fuel flow rate, $\mathrm{cf} / \mathrm{hr}$

$\mathrm{HHV}=$ Higher heating value of the fuel, Btu/cf

$\mathrm{OH}=$ Annual operating hours, $\mathrm{hr} / \mathrm{yr}$

EFFc = Current efficiency of boiler, no units

$\mathrm{EFFp}=$ proposed efficiency of the boiler, no units

For boiler \#7, the combustion efficiency calculated is $80.02 \%$ as from the boiler efficiency study. So, if we take an increase in efficiency due to the replacement of the burner on this boiler to be $2 \%$, then the new efficiency of the boiler is $82.02 \%$.

$$
\begin{array}{ll}
\mathrm{U} & =[(33,246 * 1,090 * 8,760)+(138,430 * 322 * 8,760)] / 1,000,000 \\
& =707,918 \mathrm{MMBtu} / \mathrm{yr} \\
& =707,918 *(1-0.8002 / 0.8202) \\
& =17,262 \mathrm{MMBtu} / \mathrm{yr}
\end{array}
$$

This calculation is done for the various boilers and the total annual cost savings obtained is $\$ 362,955$ from all the burners. The total annual energy savings obtained is 74,836 MMBtu, which gives a $\mathrm{CO}_{2}$ emission of 8,456,468 lb/yr.

\subsubsection{Compressor}

There are four main centrifugal compressors that have operating capacities of 2,500, 2,500, 2,500 and 3,000 scfm respectively. These compressors feed the entire plant's compressed air system for instrumentation and process needs. The air system is a complex maze of piping with many tie-in points and runs for piping. The compressors range in age from 13 to 30 years. Centralized monitoring of these units and metering of usages are not currently available. Also AC \#3 and AC \#4 have an inlet-throttling device, which have a throttling capacity of $70 \%$. There is no throttling available for AC \#1 and AC \#2.

As per the discussions with the utility department personnel, the plant instrumentation and process requires on an average a constant supply of $6600 \mathrm{scfm}$ of compressed air supply in the plant. Also one of the four air compressors is always used as a stand by, so the remaining 3 units fulfill the demand of compressed air. Also it came up 
in the discussions that the AC \# 3 will be shortly taken out of service due to the probable close down of some units in the plant. So, all our efforts in optimizing the compressed air system were centered on AC \#1, AC \#2 and AC \#4 in the plant. From the data collected over two complete shifts in the plant, the AC \#1 is almost completely loaded to its rated current during the operation with an average load factor of 95\%. Similar, conditions can be assumed to be applicable for the AC \#2. Also as seen in the existing arrangement Table 3.14 below, AC \#4 vents 500 scfm of compressed air due to the demand and supply imbalance. This $500 \mathrm{scfm}$ of air is vented the inlet throttle capacity is used to its maximum. As a result, AC \#4 is loaded up to $80 \%$. With this existing arrangement, we have an unnecessary wastage of compressed air of around $500 \mathrm{scfm}$.

\begin{tabular}{|c|c|c|c|c|c|}
\hline Compressor & $\begin{array}{c}\text { Rated } \\
\text { Power }\end{array}$ & Rated & $\begin{array}{c}\text { Inlet } \\
\text { Throttle }\end{array}$ & Current & $\begin{array}{c}\text { Amount } \\
\text { Vented }\end{array}$ \\
\cline { 2 - 6 } & $\mathbf{( k W )}$ & $\mathbf{S c f m}$ & & Scfm & Scfm \\
\hline AC \# 1 (centrifugal) & 447 & 2500 & NO & 2500 & 0 \\
\hline AC \# 2 (centrifugal) & 447 & 2500 & NO & 2500 & 0 \\
\hline AC \# 3 (centrifugal) & 521.5 & 3000 & Up to 70 \% & - & - \\
\hline AC \# 4 (centrifugal) & 596 & 3000 & Up to 70 \% & 2100 & 500 \\
\hline
\end{tabular}

\section{Table 3.14: Existing arrangement of the compressors}

The recommendation in this area was to use a reciprocating compressor in the place of the AC \#4 centrifugal compressor. The other two centrifugal compressors AC \#1 and AC \#2 can be used to base load the demand. The new reciprocating compressor can fulfill the remaining demand of $1600 \mathrm{scfm}$. This new reciprocating compressor delivers this required volume at required pressure, but at nearly half the rated power. The motor connected to this compressor would be rated to around $400 \mathrm{hp}$ (this is obtained after consulting with several reciprocating compressor manufacturers in USA), which is half the size of the current AC \#4 (800 hp). This arrangement would give a better load factor and there won't be any wastage of compressed air at all from the system. Please refer to the proposed arrangement of compressors in the following Table (Table 3.15).

\begin{tabular}{|c|c|c|c|c|c|}
\hline Compressor & $\begin{array}{c}\text { Rated } \\
\text { Power }\end{array}$ & Rated & $\begin{array}{c}\text { Inlet } \\
\text { Throttle }\end{array}$ & Current & \begin{tabular}{c} 
Amount \\
Vented \\
\cline { 2 - 6 }
\end{tabular} \\
$\mathbf{( k W )}$ & Scfm & & Scfm & Scfm \\
\hline AC \# 1 (centrifugal) & 447 & 2500 & NO & 2500 & 0 \\
\hline AC \# 2 (centrifugal) & 447 & 2500 & NO & 2500 & 0 \\
\hline AC \# New (Reciprocating) & 298 & 2000 & NO & 1600 & 0 \\
\hline
\end{tabular}

Table 3.15: Proposed arrangement 
The savings obtained from this recommendation are in the form of the demand and usage charge saved, by installing a smaller size reciprocating type compressor with a rated capacity of 2,000 scfm and 125 psig.

Demand cost $\quad=\mathrm{R}_{\mathrm{D}} * \mathrm{P}_{\text {rated }} * 12$

Usage cost $\quad=\mathrm{R}_{\mathrm{u}} * \mathrm{P}_{\text {rated }} * \mathrm{LF} * \mathrm{UF} * \mathrm{hrs} / \mathrm{yr}$

Total annual cost $\quad=$ Demand cost + Usage cost

Where,

$$
\begin{aligned}
& \mathrm{R}_{\mathrm{D}}=\text { Demand Rate }(\$ / \mathrm{kW}) \\
& \mathrm{R}_{\mathrm{u}}=\text { Usage Rate }(\$ / \mathrm{kWh}) \\
& \mathrm{P}_{\text {rated }}=\text { Rated power }(\mathrm{kW}) \\
& \mathrm{LF}=\text { Average load facto } \\
& \mathrm{UF}=\text { Average utilization factor }
\end{aligned}
$$

Using these formulae, the cost of compressed sir system in the existing and the proposed system is calculated as show in the Table's 3.16 and 3.17. The demand rate is $7.72 \$ / \mathrm{kW}$ for on peak, while the usage rate is $0.0224 \$ / \mathrm{kWh}$. The operating hours are assumed to be $8760 / y r$ with a utilization factor of 0.9 .

\begin{tabular}{|c|c|c|c|c|c|c|}
\hline Compressor & $\begin{array}{c}\text { Rated } \\
\text { Power }\end{array}$ & $\begin{array}{c}\text { Amount } \\
\text { Vented }\end{array}$ & $\begin{array}{c}\text { Load } \\
\text { Factor }\end{array}$ & $\begin{array}{c}\text { Demand } \\
\text { Cost }\end{array}$ & $\begin{array}{c}\text { Usage } \\
\text { Cost }\end{array}$ & $\begin{array}{c}\text { Total } \\
\text { Cost }\end{array}$ \\
\cline { 2 - 7 } & $\mathbf{( k W )}$ & $\mathbf{( S c f m )}$ & $\mathbf{\%}$ & $\mathbf{( \$ / y r )}$ & $\mathbf{( \$ / y r )}$ & $\mathbf{( \$ / y r )}$ \\
\hline AC \# 1 (centrifugal) & 447 & 0 & 95 & 41,410 & 74,993 & 116,403 \\
\hline AC \# 2 (centrifugal) & 447 & 0 & 95 & 41,410 & 74,993 & 116,403 \\
\hline AC \# 4 (centrifugal) & 596 & 500 & 80 & 55,213 & 84,203 & 139,417 \\
\hline \multicolumn{7}{|l|}{}
\end{tabular}

Table 3.16: Annual cost of the existing system

\begin{tabular}{|c|c|c|c|c|c|c|}
\hline Compressor & $\begin{array}{c}\text { Rated } \\
\text { Power }\end{array}$ & $\begin{array}{c}\text { Amount } \\
\text { Vented }\end{array}$ & $\begin{array}{c}\text { Load } \\
\text { Factor }\end{array}$ & $\begin{array}{c}\text { Demand } \\
\text { Cost }\end{array}$ & $\begin{array}{c}\text { Usage } \\
\text { Cost }\end{array}$ & $\begin{array}{c}\text { Total } \\
\text { Cost }\end{array}$ \\
\cline { 2 - 7 } & $\mathbf{( k W )}$ & Scfm & $\mathbf{\%}$ & $\mathbf{( \$ / y r )}$ & $\mathbf{( \$ / y r )}$ & $\mathbf{( \$ / y r )}$ \\
\hline AC \# 1 (centrifugal) & 447 & 0 & 95 & 41,410 & 74,993 & 116,403 \\
\hline AC \# 2 (centrifugal) & 447 & 0 & 95 & 41,410 & 74,993 & 116,403 \\
\hline AC \# New (Reciprocating) & 298 & 0 & 95 & 27,606 & 49,995 & 77,602 \\
\hline \multicolumn{7}{|l|}{}
\end{tabular}

Table 3.17: Annual cost of the proposed system 
Savings obtained $=$ Annual cost of existing system - Annual cost of proposed system

$$
\begin{aligned}
& =\$ 372,224-\$ 310,410 \\
& =\$ 61,814 .
\end{aligned}
$$

Therefore the annual savings obtained by implementing the proposed recommendation is $\mathbf{\$ 6 1 , 8 1 4}$. Energy will be saved in the form of demand savings (DS) in $\mathrm{kW}$ and the electricity or usage energy savings (ES) in kWh.

$$
\begin{aligned}
\text { DS } & =\text { Current total demand }- \text { Proposed total demand } \\
& =1,490 \mathrm{~kW}-1,192 \mathrm{~kW} \\
& =298 \mathrm{~kW} \\
\text { ES } & =\text { Current electricity usage }- \text { Proposed electricity usage } \\
& =3,759,091 \mathrm{kWh}-2,231,960 \mathrm{kWh} \\
& =1,527,131 \mathrm{kWh} * 3,413 / 10^{6} \mathrm{MMBtu} \\
& =5,212 \mathrm{MMBtu}
\end{aligned}
$$

$$
\begin{aligned}
\mathrm{CO}_{2} \text { emissions } & =1,527,131 \mathrm{kWh} \text { X } 2.19 \mathrm{lbs} / \mathrm{kWh} \\
& =3,344,417 \mathrm{lbs}
\end{aligned}
$$

The implementation cost of this recommendation involves a new set of reciprocating compressor system. The old centrifugal compressor \#4 can be salvaged at an estimated value of $\$ 35,000$. The cost of a new reciprocating compressor would be approximately \$200,000 including installation and other charges. (The salvage value and the implementation cost are obtained by consultation with many vendors). So the net implementation cost can be assumed to be:

Net implementation cost $\quad=$ Total cost for implementing reciprocation compressor Salvage value of the centrifugal compressor

$$
\begin{aligned}
& =\$ 200,000-\$ 35,000 \\
& =\$ 165,000 .
\end{aligned}
$$

Simple payback can be calculated as follows:

Payback period (months) = Savings $/$ Net implementation cost $* 12$ 


$$
\begin{aligned}
& =\$ 165,000 / \$ 61,814 * 12 \\
& =32 \text { months }
\end{aligned}
$$

\subsubsection{Condensate Return}

In the case of condensate return back to the boiler, there are two components to the annual savings involved. They are fuel savings, and water and chemical Savings. Water and chemical savings involves the cost of the makeup water and the chemicals that are used for treating them. The fuel savings represents the amount of heat energy that is saved in heating the water to the temperature required to convert it to steam. Currently thirty percent of the condensate is being returned back to the boiler. Calculations are done for the various percentages of condensate return to give an idea as to how much of savings can be obtained by the return of varying amounts of condensate back to the boiler system. A practical amount of return that can be achieved is around seventy-five percent which can lead to a savings in cost of around $\$ 375,000$ for an approximate implementation cost of $\$ 100,000$.

\subsubsection{Adjustable Speed Drive on the Cooling Tower Pump}

A variable speed drive is an electronic device that controls motor speed by varying the frequency of the electrical supply. Our recommendation was to install variable speed drive (VSD) on all the pump motors of the some of the cooling tower systems. This would reduce pumping energy costs when one or more of the process side users are not operating. Eight cooling tower systems operated by the utilities department supply tower water to designated users. Cooling tower performance is weather dependent and restricts production in some areas during hot, humid weather. Several operating areas are supplied from the same tower. This restricts maintenance efforts. Towers range from 17 to over 30 years old. Metering of tower water flows to users does not exist. Monitoring and control of tower water is available only on a limited local basis. Schematic of the current cooling system is shown in Figure 3.8. 


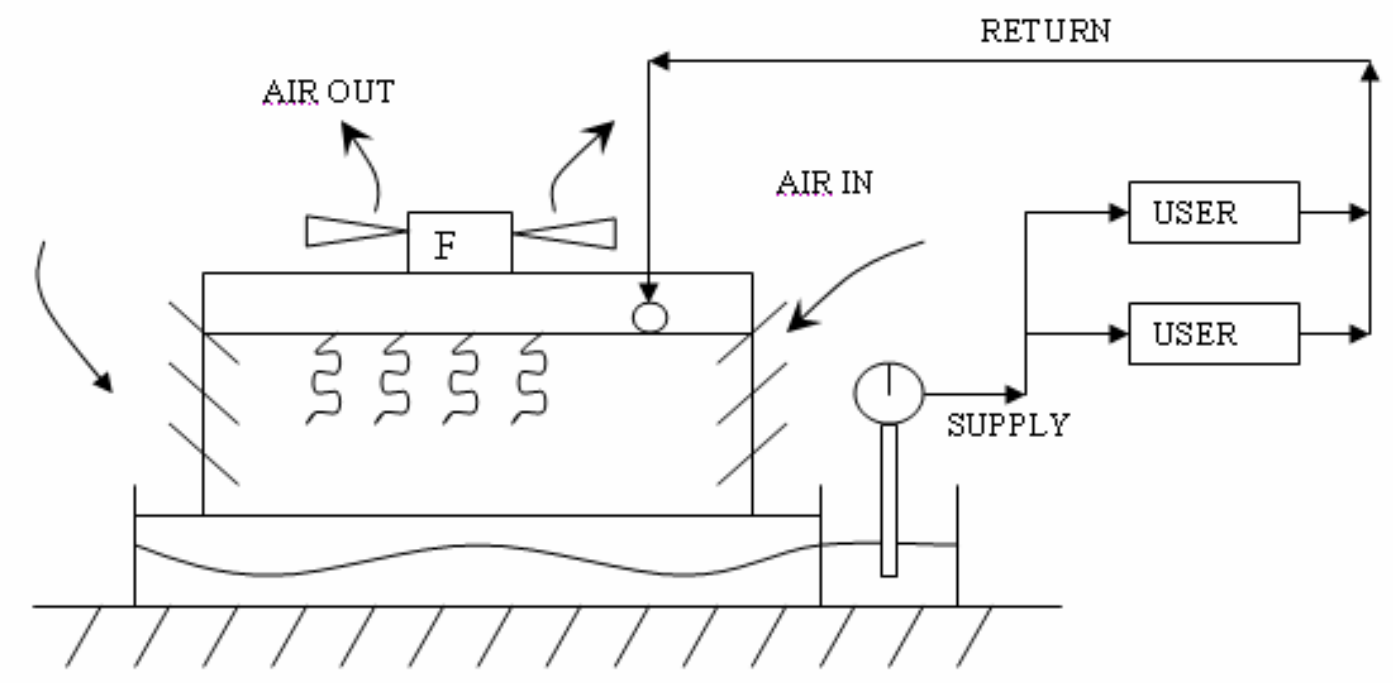

Figure 3.8: Tower Water Generation and Distribution

However, according to the discussions with the utilities department, at a given time not more than $60 \%$ of the users connected to each cooling tower station are operating at the same time. Since the motor connected to the pump is constant speed, the cooling water to each user is kept running even if the user is not operating. This creates an opportunity for installing variable speed drive (VSD) on the cooling water supply pump. Since peak conditions typically occur infrequently, substantial energy savings are possible by varying fluid flow rate to match the actual demand. Although the fluid flow rate can be controlled by varying the system pressure drop with dampers, valves and inlet vanes, energy savings are much greater if the flow is controlled by varying the speed of the pump or fan with an electronic variable speed drive (VSD). In addition to substantial energy savings, other advantages of VSD's include smoother control, softer starts, reduced noise, reduced damper/valve maintenance, reduced flow friction heating, and faster response. It is recommended that the pumps on the cooling tower number 1, 2, 10 and 12 are appropriate for an initial VSD application.

Following is the sample calculation for the Cooling Tower \# 1 pumps. There are 3 pumps on this cooling tower, namely, east pump, center pump and west pump. During field audit and from discussions with the utilities department, it is found that on an average the 75-hp pump motors is $75 \%$ loaded and $91 \%$ efficient. If so, the power drawn for cooling pump motor would be: 
Power drawn = Rated power $(\mathrm{HP}) \times 0.745 \times \mathrm{LF} /$ Eff.

Where,

$$
\begin{aligned}
& \text { LF = load factor } \\
& \text { Eff. = efficiency of the motor }
\end{aligned}
$$

Power drawn = 75 hp x $0.745 \mathrm{~kW} / \mathrm{hp} \times 75 \% / 91 \%$

$$
=46.05 \mathrm{~kW}
$$

When estimating power savings from reducing the volume flow rate, engineers frequently rely on pump/fan affinity laws. Theoretically, pump/fan work varies with the cube of volume flow rate. In practice, however, control, VSD efficiency and pump efficiency losses result in slightly less savings than would be predicted using this 'cubic' relationship. Thus, we conservatively estimate that pump/fan work varies with the square of flow rather than the cube of flow. Based on the loading, electrical saving from installing a VSD when $60 \%$ of the users are operating would be about:

$$
\mathrm{P}_{2}=\mathrm{P}_{1}\left(\mathrm{~V}_{2} / \mathrm{V}_{1}\right)^{2}=46.05 \mathrm{~kW} \times(60 \%)^{2}=16.58 \mathrm{~kW}
$$

This would give a slightly conservative estimate of savings. Therefore, the electrical savings for the 8,760 annual operating hours would be as below.

$$
\begin{aligned}
& \left.\begin{array}{l}
\text { Demand savings } \\
\text { Savings in } \mathrm{kW}
\end{array}\right\}=(46.05-16.58) \mathrm{kW}=29.47 \mathrm{~kW} \\
& \text { Demand savings } \\
& =29.47 * 7.72 * 12 \\
& =2,730 \$ / \mathrm{yr}
\end{aligned}
$$

The annual energy savings for 8,760 operating hrs/yr would be as follows.

$$
\begin{aligned}
\text { Energy savings in } \mathrm{kWh} & =29.47 \mathrm{~kW} * 8,760 \mathrm{hrs} / \mathrm{yr} \\
& =258,157 \mathrm{kWh} \\
& =258,157 \mathrm{kWh} * 3,413 / 10^{6} \mathrm{MMBtu}
\end{aligned}
$$




$$
\begin{array}{ll} 
& =881 \mathrm{MMBtu} \\
\text { Energy savings } & =29.47 \mathrm{~kW} * 8760 \mathrm{hrs} / \mathrm{yr} * 0.0224 \$ / \mathrm{kWh} \\
& =5,782 \$ / \mathrm{yr} . \\
& =\text { Demand savings + Energy savings } \\
& =2,730.10 \$ / \mathrm{yr}+\$ 5,782.72 \$ / \mathrm{yr} \\
& =8,512 \$ / \mathrm{yr} \\
& =258,157 \mathrm{kWh} \times 2.19 \mathrm{lbs} / \mathrm{kWh} \\
\mathrm{CO}_{2} \text { emissions } & =565,364 \mathrm{lbs}
\end{array}
$$

According to a VSD manufacturer, a VSD for a 75-hp motor would cost about \$160/hp.

$$
\begin{aligned}
\text { Implementation cost } & =\$ 160 / \mathrm{hp} \times 75 \mathrm{hp} \\
& =\$ 12,000
\end{aligned}
$$

(http://www.nwalliance.org/resources/reports/E00-054.pdf)

Simple payback can be calculated as follows:

$$
\begin{aligned}
\text { Payback period } & =\$ 12,000 / \$ 8,512 \$ / \mathrm{yr} \times 12 \text { months } / \mathrm{yr} \\
& =17 \text { months. }
\end{aligned}
$$

There are 3 similar pumps on this cooling tower and thus for Cooling tower \#1, on all the three pumps the total savings achieved will be as follows:

$$
\begin{array}{ll}
\text { Total Savings obtained } & =3 \times \$ 8,512 / \mathrm{yr} \\
& =25,538 \$ / \mathrm{yr} \\
\text { Total implementation cost } & =3 \times \$ 12,000 \\
& =\$ 36,000 . \\
\text { Simple Payback } & =17 \text { months }
\end{array}
$$

Similar calculations were performed on all other cooling towers, which are candidates of ASD application. The details of which are shown as follows in Table 3.18. The total demand savings obtained are $530 \mathrm{~kW}$ and the total electricity savings obtained are $15,844 \mathrm{MMBtu}$. The $\mathrm{CO}_{2}$ emissions saved total to $10,166,773 \mathrm{lbs}$ for all the motors. It has to be noted that 2300-volt ASD applications are not widely used and need to be proven reliable. 


\begin{tabular}{|l|c|c|c|c|c|c|}
\hline \multicolumn{1}{|c|}{ Unit } & $\begin{array}{c}\text { \# of CT } \\
\text { pumps }\end{array}$ & Voltage & $\begin{array}{c}\text { Motor Size } \\
\mathbf{( H p )}\end{array}$ & $\begin{array}{c}\text { Total } \\
\text { Savings }\end{array}$ & $\begin{array}{c}\text { Implementation } \\
\text { Cost }\end{array}$ & Payback \\
\hline CT \# 1 & 3 & 480 & 75 & $\$ 25,538$ & $\$ 36,000$ & 17 months \\
\hline CT \# 2 & 3 & 480 & 75 & $\$ 25,538$ & $\$ 36,000$ & 17 months \\
\hline CT \# 10 & 3 & 2300 & 200 & $\$ 51,003$ & $\$ 96,000$ & 23 months \\
\hline CT \# 12 & 3 & 2300 & 200 & $\$ 51,003$ & $\$ 96,000$ & 23 months \\
\hline Total & & & & $\$ 153,082$ & $\$ 264,000$ & \\
\hline
\end{tabular}

Table 3.18: Summary of all VSD applications

The analysis is performed for each of the areas in the assessment plan in detail to arrive at the detailed recommendations for energy savings. Once this step is completed, the sensitivity analysis is performed on the key parameters.

\subsection{Sensitivity Analysis}

Sensitivity analysis is defined as the systematic investigation of the reaction of the simulation response to either extreme values of the model's quantitative factors (parameters and input variables) or to drastic changes in the model's qualitative factors (modules). Sensitivity analysis helps us determine the change in the output of a model when the values of the critical input factors are varied between certain limits. It helps us to determine a trend of the output based on the change in the input factor. This analysis is aimed at studying the parameters affecting energy used in the plant, by carrying out the sensitivity analysis, determining the cut-off points or the critical points of a particular factor and analyzing the trend of the parameter when they are varied against other parameters.

The sensitivity is done with respect to two most important factors of the equation that affect the annual savings obtained. Other factors like the percent savings obtained are not considered for sensitivity analysis, as it is obvious that with increase in savings percentage the energy savings will increase. This sensitivity analysis is performed by varying only one important parameter in the equation and then the impact of that varied parameter is measure and analyzed. Like wise all the parameters are varied keeping all others constant and the sensitivity analysis is performed. The key parameters are varied within a practical range for the purpose of the analysis so as to measure the impact on the energy savings obtained. 


\begin{tabular}{|c|c|c|c|c|c|c|c|c|c|}
\hline $\begin{array}{c}\text { Excess } \\
\text { Air }\end{array}$ & $\begin{array}{c}\text { Oxygen } \\
\text { \% }\end{array}$ & CO2 \% & \multicolumn{7}{|c|}{ Net Stack Temperature } \\
\cline { 3 - 9 } & & $\mathbf{2 2 0}$ & $\mathbf{2 3 0}$ & $\mathbf{2 4 0}$ & $\mathbf{2 4 6}$ & $\mathbf{2 5 0}$ & $\mathbf{2 6 0}$ & $\mathbf{2 7 0}$ \\
\hline 0 & 0 & 11.8 & 85.3 & 85.1 & 84.9 & 84.8 & 84.7 & 84.5 & 84.2 \\
\hline 2.2 & 0.5 & 11.5 & 85.2 & 85 & 84.8 & 84.7 & 84.6 & 84.4 & 84.1 \\
\hline 4.5 & 1 & 11.2 & 85.1 & 84.9 & 84.7 & 84.6 & 84.5 & 84.2 & 84 \\
\hline 6.9 & 1.5 & 11 & 85 & 84.8 & 84.6 & 84.5 & 84.4 & 84.1 & 83.9 \\
\hline 9.5 & 2 & 10.7 & 84.9 & 84.7 & 84.5 & 84.3 & 84.2 & 84 & 83.8 \\
\hline 12.1 & 2.5 & 10.4 & 84.8 & 84.6 & 84.4 & 84.2 & 84.1 & 83.9 & 83.7 \\
\hline 15 & 3 & 10.1 & 84.7 & 84.5 & 84.2 & 84.1 & 84 & 83.8 & 83.5 \\
\hline 18 & 3.5 & 9.8 & 84.6 & 84.4 & 84.1 & 84 & 83.9 & 83.6 & 83.4 \\
\hline 21.1 & 4 & 9.6 & 84.5 & 84.2 & 84 & 83.8 & 83.7 & 83.5 & 83.2 \\
\hline 24.5 & 4.5 & 9.3 & 84.3 & 84.1 & 83.8 & 83.7 & 83.6 & 83.3 & 83.1 \\
\hline 28.1 & 5 & 9 & 84.2 & 83.9 & 83.7 & 83.5 & 83.4 & 83.2 & 82.9 \\
\hline 31.9 & 5.5 & 8.7 & 84.1 & 83.8 & 83.5 & 83.4 & 83.3 & 83 & 82.7 \\
\hline 35.9 & 6 & 8.4 & 83.9 & 83.6 & 83.3 & 83.2 & 83.1 & 82.8 & 82.5 \\
\hline 40.3 & 6.5 & 8.2 & 83.7 & 83.4 & 83.2 & 83 & 82.9 & 82.6 & 82.3 \\
\hline 44.9 & 7 & 7.9 & 83.5 & 83.3 & 83 & 82.8 & 82.7 & 82.4 & 82.1 \\
\hline 49.9 & 7.5 & 7.6 & 83.4 & 83.1 & 82.8 & 82.6 & 82.5 & 82.2 & 81.9 \\
\hline 55.3 & 8 & 7.3 & 83.1 & 82.8 & 82.5 & 82.3 & 82.2 & 81.9 & 81.6 \\
\hline 61.1 & 8.5 & 7 & 82.9 & 82.6 & 82.3 & 82.1 & 82 & 81.6 & 81.3 \\
\hline 67.3 & 9 & 6.7 & 82.7 & 82.3 & 82 & 81.8 & 81.7 & 81.4 & 81 \\
\hline 74.2 & 9.5 & 6.5 & 82.4 & 82.1 & 81.7 & 81.5 & 81.4 & 81 & 80.7 \\
\hline 81.6 & 10 & 6.2 & 82.1 & 81.8 & 81.4 & 81.2 & 81.1 & 80.7 & 80.3 \\
\hline 89.8 & 10.5 & 5.9 & 81.8 & 81.4 & 81.1 & 80.9 & 80.7 & 80.3 & 79.9 \\
\hline 98.7 & 11 & 5.6 & 81.5 & 81.1 & 80.7 & 80.5 & 80.3 & 79.9 & 79.5 \\
\hline 108.7 & 11.5 & 5.3 & 81.1 & 80.7 & 80.3 & 80.1 & 79.7 & 79.4 & 79 \\
\hline 119.7 & 12 & 5.1 & 80.6 & 80.2 & 79.8 & 79.4 & 79.4 & 78.9 & 78.5 \\
\hline
\end{tabular}

Table 3.19: Boiler Efficiency Sensitivity Analysis

Efficiency $(\%)=100-$ SensibleHeatLoss - LatentHeatLoss

SensibleHeatLoss $=(T-t) \times\left[0.023+0.00011\left(\% O_{2}+1\right)^{2}\right]$

LatentHeatLoss $=9$ (gas)

$$
=5 \text { (oil) }
$$

Where,

$$
\begin{aligned}
& \mathrm{T}=\text { Flue gas temperature, }{ }^{\circ} \mathrm{F} \\
& \mathrm{t}=\text { Air dry bulb temperature, }{ }^{\circ} \mathrm{F}
\end{aligned}
$$




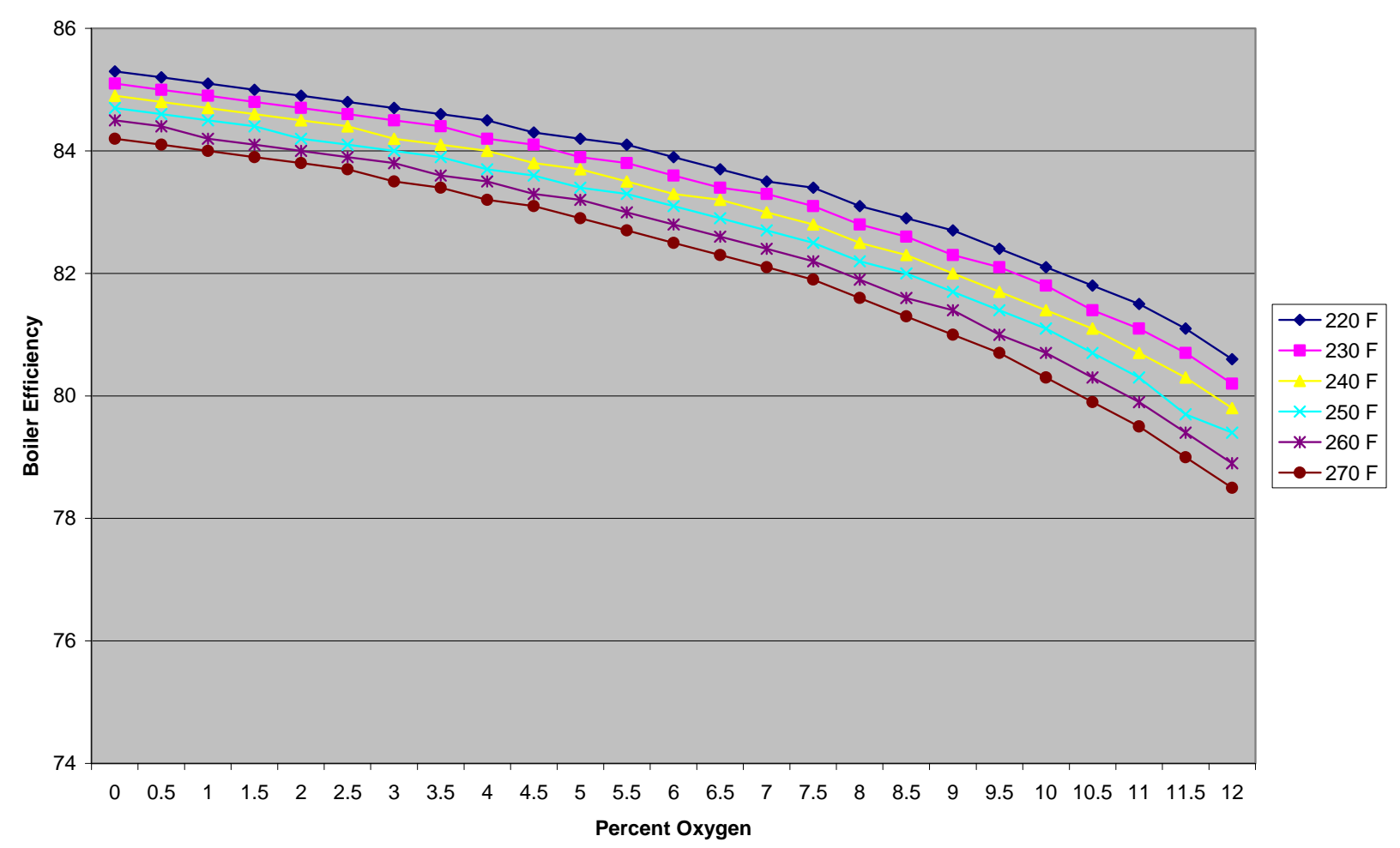

Figure 3.9: Boiler Efficiency Sensitivity

This graph can be used as a quick reference for estimating the efficiency of a natural gas firing boiler. It helps the operator to do a quick analysis of the boiler settings to maintain optimal efficiency of the boiler. It can be seen from the graph that as the flue gas temperature increases, the boiler efficiency decreases. The efficiency decreases gradually for increased percentages of oxygen up to around seven percent, beyond which the efficiency drops rapidly. This is because higher percentages of oxygen causes increased amounts of hot gases to be lost in the stack. Heat is absorbed by the air instead of being transferred to output, and efficiency falls [48]. With high excess oxygen levels, some fuel is used to heat unneeded excess air to the stack temperature. As greater amounts of excess air used, the combustion gases pass through the boiler heat transfer areas very quickly thereby retaining their heat, rather than transferring it to the water in the tubes. Hence, the overall efficiency of the boiler drops as is indicated by the formula shown.

The following analysis is done for various insulation materials at different thickness of insulation. In many cases, it may not be practical to have more than six inches of 
insulation on a pipe or surface. The analysis was done for four different insulation materials for the same operating conditions, to determine the best insulation material for the application. The materials that were analyzed were $850^{\circ} \mathrm{F}$ mineral fiber blanket, cellular glass pipe, mineral fiber metal mesh blanket, and mineral fiber insulating cement. The following table shows the heat loss for various thickness of insulation material for $850^{\circ} \mathrm{F}$ mineral fiber blanket (ASTM C553-00 T4). The values for the heat loss were obtained from the 3E Plus ${ }^{\circledR}$ insulation software for the different thickness of insulation materials.

\begin{tabular}{|c|c|c|c|}
\hline \multicolumn{3}{|c|}{ Material = 850F MF BLANKET ASTM C553-00 } & \multirow{2}{*}{\begin{tabular}{|c|} 
T4 \\
$\begin{array}{c}\text { Efficiency } \\
(\%)\end{array}$
\end{tabular}} \\
\hline Thickness & $\begin{array}{c}\text { Surface } \\
\text { Temp }(-F)\end{array}$ & $\begin{array}{l}\text { Heat } \\
\text { Loss }\end{array}$ & \\
\hline 0 & 499 & 1029 & \\
\hline 0.5 & 260 & 206.7 & 79.91 \\
\hline 1 & 178 & 131.9 & 87.18 \\
\hline 1.5 & 146 & 104.1 & 89.88 \\
\hline 2 & 129 & 88.98 & 91.35 \\
\hline 2.5 & 118 & 79.45 & 92.28 \\
\hline 3 & 111 & 72.6 & 92.94 \\
\hline 3.5 & 105 & 67.42 & 93.45 \\
\hline 4 & 100 & 62.83 & 93.89 \\
\hline 4.5 & 96.9 & 59.66 & 94.2 \\
\hline 5 & 94.1 & 56.96 & 94.46 \\
\hline 5.5 & 92.5 & 55.36 & 94.62 \\
\hline 6 & 91.4 & 54.36 & 94.72 \\
\hline 6.5 & 88.6 & 51.49 & 95 \\
\hline 7 & 87.2 & 49.92 & 95.15 \\
\hline 7.5 & 85.7 & 48.52 & 95.28 \\
\hline 8 & 84.6 & 47.26 & 95.41 \\
\hline 8.5 & 83.5 & 46.13 & 95.52 \\
\hline 9 & 82.6 & 45.11 & 95.62 \\
\hline 9.5 & 81.7 & 44.17 & 95.71 \\
\hline 10 & 80.9 & 43.31 & 95.79 \\
\hline
\end{tabular}

\section{Table 3.20: Sensitivity of Insulation Material Type}

From the analysis we find that the mineral fiber metal mesh blanket gives a better insulation compared to that of the other materials, with mineral fiber insulating cement giving the least effect (Figure 3.8). We find that the initial two inches of insulation give more reduction in heat loss and increased diameters give lesser reductions. This is because the surface exposed to the ambient increases as the diameter of the pipe with insulation increases and hence, it has better heat loss from the surface. The heat loss is a function of 
the pipe size and insulation quality, the steam temperature (pressure) and the surrounding temperature.

Effect of Various Insulation Materials on Heat Loss

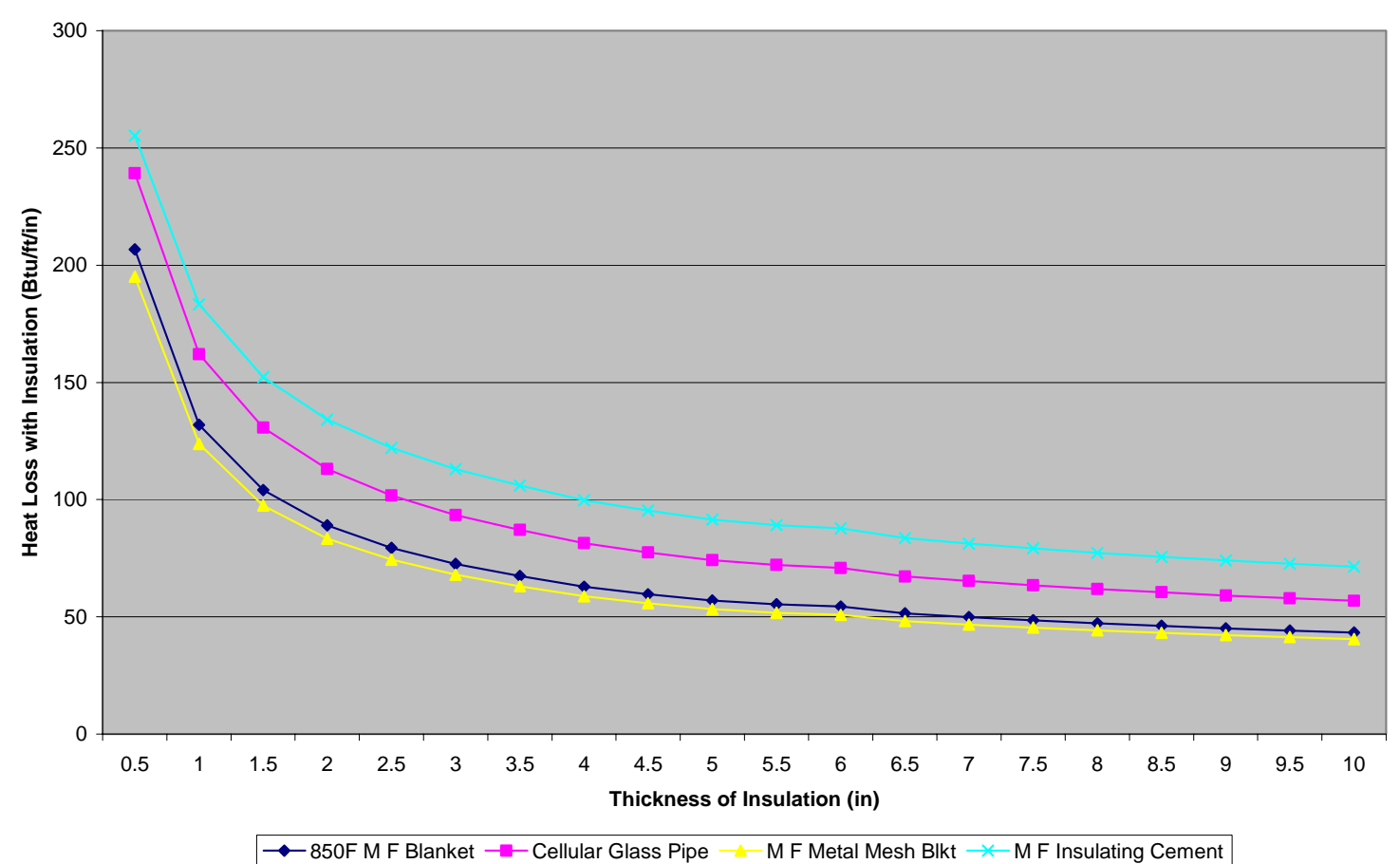

Figure 3.10: Insulation Material Sensitivity

Heat flow per foot of pipe with an insulating jacket over the pipe $\left(U_{p}\right)$ is [49]:

$$
U_{p}=\frac{0.524 k \Delta T r_{2}}{r_{2} \ln \left(\frac{r_{2}}{r_{1}}\right)+k R_{s}}
$$

Where,

$\mathrm{k}=$ Insulation thermal conductivity at the mean temperature across the insulation

$\Delta \mathrm{T}=$ Temperature difference between the ambient and the fluid in the pipe

$\mathrm{R}_{\mathrm{s}}=$ Surface resistance through which the heat must flow

$\mathrm{r}_{1}=$ Inside radius of pipe without insulation

$r_{2}=$ Outside radius of pipe with the insulation jacket. 
From the equation shown, it seen that as we increase the thickness of insulation, the $r_{2}$ value increases for the same $r_{1}$. With all the other values constant, we find that the natural logarithmic value increases for increase in thickness of insulation thereby increasing the denominator value. Hence, the heat loss through the insulated pipe goes down and with further insulation the increase in thickness just contributes to marginally more surface area exposed to the ambient and hence, the heat loss does not drop down drastically.

The next analysis involves the effect of the diameter of the steam leaks to the annual energy loss. The diameter was determined as a factor that can be varied because if the leak is not fixed immediately, the leak diameter increases with the course of time. Steam leaks are a direct loss of energy from the system and are similar to the venting of steam from the system. From the data obtained from the Energy Efficiency Handbook, the steam loss for the various diameters of leaks is as shown in Table 3.21 for a steam pressure of 650 psi.

\begin{tabular}{|c|c|}
\hline Hole Size (in) & Steam Loss (lb/hr) \\
\hline $1 / 16^{\prime \prime}$ & 86 \\
\hline $1 / 8 "$ & 345 \\
\hline $1 / 4 "$ & 1,381 \\
\hline $1 / 2^{\prime \prime}$ & 5,486 \\
\hline $1 "$ & 22,054 \\
\hline
\end{tabular}

\section{Table 3.21: Steam Leaks Sensitivity}

It can be seen from the figure (Figure 3.11) that the steam loss increases drastically once the diameter becomes more the $1 / 4$ th inch. This suggests that the steam leaks should be fixed in the shortest possible time in order to avoid expensive repairs and increased fuel costs. The increase in the steam loss is dependant on the pressure of the steam flowing through the pipe and on the size of the hole through which the leak occurs. 
Steam Leaks

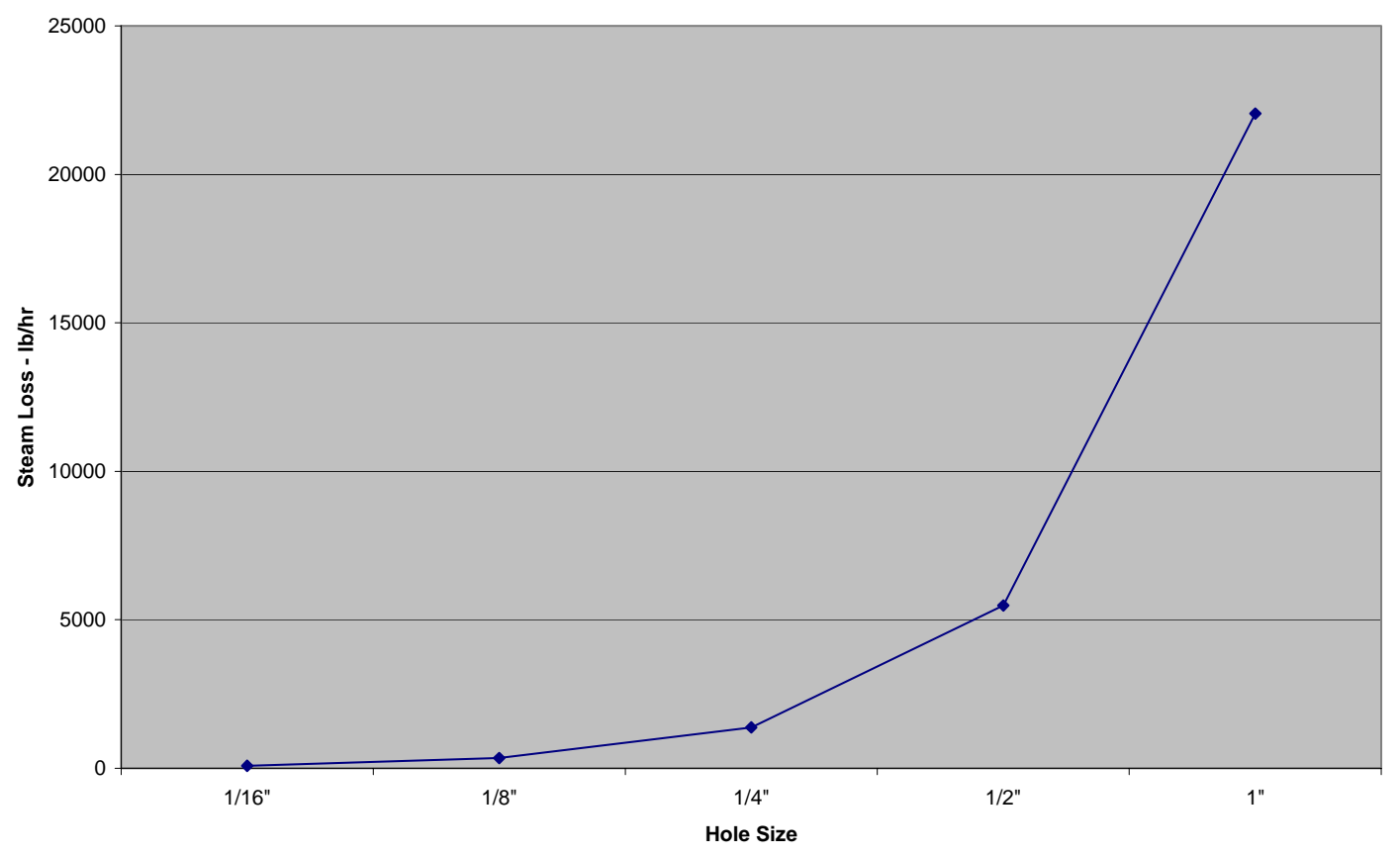

Figure 3.11: Steam Leaks Sensitivity to Varying Diameters

\subsection{Final Report and Corporate Analysis}

Once the detailed data analysis and the sensitivity analysis on the key parameters are completed, the results are compiled together to produce a report that can be presented to the plant personnel for review. The report has the recommendations detailing the economic analysis and the technical report with the collected data. The economic analysis section of the report has a concise summary of the various recommendations in the report along with their energy and cost savings, and their implementation costs. This section is mainly useful for the corporate people to get an idea of the savings involved in implementing a recommendation. The next section which is the technical section is mainly for the plant maintenance personnel. The section explains the complete information on the data collected for every area, the recommendation to produce energy savings, and the economics involved. It also identifies the best practices followed in each area, the maintenance problems found and the tips for regular maintenance in that area. The final report also gives information on studies of efficiency of certain systems and the recommendations that are not economically feasible to implement. 
The final report is compiled together and reviewed until no errors are found by the assessment team. It is then presented to the plant personnel for their review. Some of the data that is presented in the report might be confidential to the company and they might need it to be in some particular format. Once the reviews are completed, the information is presented to the corporate people in the plant for their approval. A brief overview of the project and the various recommendations are presented to them. The interaction that is continuously maintained with the plant personnel helps to make sure that there are no huge errors that surface during the reporting process.

\subsection{Implementation and Continuous Improvement}

Once the corporate people approve the final report, the implementation process could be commenced for the various recommendations. The plant gives the approval for the implementation of the recommendations subject to availability of capital and the attractiveness of payback on investment. Technical meetings will be held in the plant to discuss the recommendations generated by the plant-wide assessment and action plans for implementation will be developed.

To ensure continuous improvement in the management and use of raw materials in the generation of utilities it is important that specific equipment be set at optimal conditions so as to ensure maximum possible efficiency. In some cases, such as in hydrogen and natural gas burning boilers, this concept has not yet been deeply investigated. In our assessment, boilers and associated steam systems, compressor systems, and electrical motor driven pump systems are all excellent candidates for applying the principles of Optimal Equipment Efficiency (OEE). This leads to improved productivity with the utilities personnel being able to quickly set the equipment to operate at predetermined levels as required at various points in time, eliminating the guess work involved in maintaining equipment at peak operating efficiencies. In our assessment, a spreadsheet was developed to calculate the efficiency of a boiler firing natural gas and hydrogen. This helps the plant personnel to get a quick estimate of the efficiency of the boiler system for a given set of operating conditions. Also a study was done on the various control methods available to obtain efficient boiler loading rather than having all the boilers loaded at part load all the time. 


\subsection{System Validation}

The systems methodology could be applied both to large facilities and for assessing smaller facilities. When applying the systems methodology to smaller facilities, few of the steps could be skipped as it will not be practical to look at them for the time available to perform the assessment.

The methodology for performing an assessment in a smaller facility is illustrated by the following figure (Figure 3.12). Initially the assessment team gains the knowledge and expertise required to perform the assessment by receiving training through seminars and by reviewing the earlier assessment reports. Then, data is collected from the plant for historic usage data on the utility bills and cost data on various utilities used in the facility. Then, this data is analyzed to arrive at the cost per unit of energy used and also, any seasonal patterns in the energy usage are analyzed. The site visit is then performed to collect onsite data for performing the various steps in the data analysis. Initially, discussions are held with the plant personnel to discuss about the assessment and to get information on any energy saving opportunity that the personnel might have. Once the initial discussions are completed, a plant walk-thru is performed, starting from the place where the raw material for the processes comes in at the docks. The assessment team follows the flow of materials through the various processes that convert it to the final product to be shipped at the shipping dock. During the walk-thru in the plant, the various processes are discussed in detail with the plant personnel. The team looks for assessment opportunities while they walk through the plant. Once the walk-thru is completed, the team makes a list of the various assessment opportunities that are identified in each of the various processes in the plant. The data analysis modification step is not performed in detail as usually for a smaller facility the main data collected is the utility bills and the analysis would not require much modification. For each of the assessment opportunities identified in the assessment plan, the team collects the required information on data gathering templates that are developed using the appropriate instrumentation. 


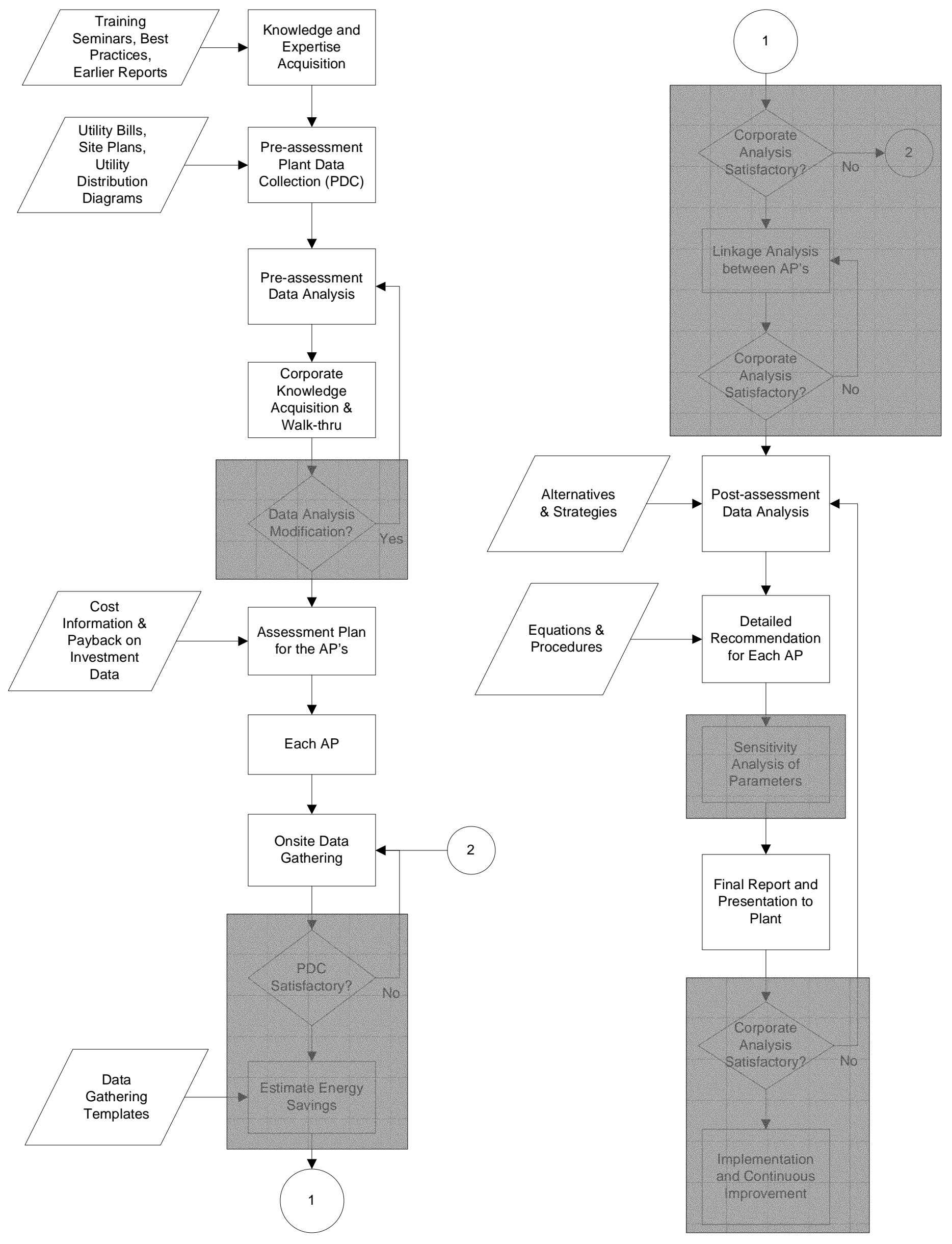

Figure 3.12: Methodology for Smaller Facilities (excluding the shaded areas) 
The next few steps that would normally be performed for a large facility are not performed because of the amount of data collected and are mainly dependant on the size of the facility. The corporate analysis step is performed to make sure that the data collected by the team is illustrative of the annual operating conditions of the plant. The operating schedules are noted for each of the various equipments. Also, before leaving the plant, the various opportunities found are discussed with the plant personnel to check for their viability for implementation. Once the team is back from the assessment, the data collected in the plant is analyzed in detail using the formulae and procedures from the earlier assessments. The various alternatives and strategies for saving energy are analyzed and detailed recommendations are developed for each of the assessment points in the assessment plan. Some of the opportunities might get dropped because of the economic insignificance or their payback periods. The various individual recommendations are compiled together to form a final report which is then presented to the plant for review and approval. The team does not get involved with the implementation of the project and after six months from the date of assessment, the team gets back with the plant personnel to find out about the various assessment points that were implemented by the plant. This step helps in tuning the cost data that is used by the plant and also, to make sure that all the formulae and procedures used in the calculations are accurate.

As seen from the description, it is evident that the methodology developed could be applied both to small and large facilities. In smaller facilities, a few of the steps are not fully performed or they are skipped to account for the lack of time and effort required to perform them in a smaller facility.

\subsection{Conclusion}

The main focus of this chapter is to explain the methodology for plant-wide energy assessments in detail. This serves as a guideline for performing plant-wide energy assessments. It describes the assessment process and the sensitivity analysis performed in each of the selected areas. The sensitivity analysis helps the equipment operators to get an easy idea as to whether the various systems are being operated at the optimal efficiency levels possible. 


\section{Conclusion and Future Work}

The main objectives of the plant-wide energy assessments are to analyze the energy use of the facility, identify areas for more detailed analysis, identify energy saving opportunities and provide a detailed analysis of the same, and help in developing the best energy strategy for the facility. The main purpose for this research work is to create a standard methodology for performing plant-wide energy assessments. It gives a detailed overview of plant-wide energy assessments, their scope and the steps involved in getting them done, and the disadvantages if a systems approach is not followed.

From the research work done so far, it can be seen that the plant-wide energy assessments provide an excellent opportunity for realizing potential energy savings in a facility, by implementing the best energy management practices for that industry including the adoption of new, efficient technologies. It can help in improving the overall energy efficiency of the systems in the plant. The results that are obtained in the energy assessment of a plant can be applied in other plants of the same organization or similar plants across the country. This helps in multiplying the savings that are obtained. Also, the results that are obtained in an energy assessment are analyzed to obtain benchmarks for energy usage called BestPractices, for various systems. These benchmarks help other industries to check their energy usage for similar operating conditions and make changes to their existing systems to achieve higher efficiency settings. Since the plant-wide energy assessments are cost-shared by the DOE, the plants can realize very good savings from energy costs, waste reduction and productivity improvements, for a relatively low initial investment.

A systematic approach should be used when performing plant-wide energy assessments. The energy usage is determined for each of the manufacturing processes in the plant and the processes that have high annual savings potential are targeted for further evaluation during the assessment. The targeted areas are then analyzed to quantify the potential energy savings and to estimate the costs involved for the implementation of the energy saving projects. If the systems approach is not used when the assessment is performed, it leads to problems in the flow of the assessment and completing it on time. In the systems approach, when the assessment is started, an assessment plan is created highlighting the time duration for each process and also ways for ensuring that 
comprehensive data is collected for each area, before the analysis is shifted to the next area. Using the systems approach, the efficiency with which the team performs the assessment is increased. This is because of the fact that the team does not waste any time due to redundant data gathering processes and also, the focus of the assessment is only on the areas that would potentially give the higher energy savings. The systems approach makes extensive use of the corporate people in the facility. This is the core of the approach as the plant personnel have complete knowledge of their facility and involving them in the assessment process helps in achieving the complete potential of the assessment. It helps in uncovering areas that would normally be overlooked, avoiding mistakes in the data collection process, and ensuring that proper data is used. Keeping the plant personnel up-to-date on the progress of the assessment helps in timely implementation of the potential energy saving projects by the plant.

This research created a standard methodology for performing plant-wide energy assessments and explains each step in detail with actual experience in a plant-wide energy assessment performed by the author. The following future work is suggested in this research:

A Computer - integrated approach to the assessment process

A Implementation - based effectiveness analysis 


\section{References}

1. Nagarajan, Sai V., "Design and Development of Expert Systems for Energy Conservation in Manufacturing Industries”, West Virginia University, 1995.

2. Mate, Amol, "Energy Analysis and Diagnostics in Wood Manufacturing Industry”, West Virginia University, 2002.

3. United States Department of Energy, Energy Information Administration, "Manufacturing Energy Consumption Survey”, http://www.eia.doe.gov/emeu/mec s/mecs94/consumption/mecs5.html , 2000.

4. Crawley, Gerard, “Energy”, Macmillan Publishing Co. Inc., p298, 1975.

5. United States Department of Energy, Energy Information Administration, "Energy in the United States: 1635 - 2000”, www.eia.doe.gov, 2000.

6. Boiler Steam Distribution, http://www.aep.com/EnergyInfo/ces_html/hstbs.htm

7. Thumann, Albert, “Handbook of Energy Audits”, The Fairmont Press, Inc., 1979.

8. Best Practices Brochure, Office of Industrial Technologies, Department of Energy, 2001.

9. Goyal, O. P., “Guidelines Help Energy Engineers And Managers”, Hydrocarbon Processing, February 2001, Vol. 80, Issue 2.

10. Oak Ridge National Laboratory, "Plant-wide Energy Assessment Solicitation", UT-Battelle for the Department of Energy, June 2001.

11. U. S. Department of Energy, "Instructions for Energy Auditors", National Technical Information Service, Springfield, Virginia, Vol. I, Sep 1978.

12. Warfel, Christopher, "An Energy Audit Method for Utilities and Industry", Energy Engineering, Vol. 90, No. 2, 1993.

13. Gary Spanner, Daryl Brown, Gregory Sullivan, Sheila Riewer, "Impact Evaluations of Industrial Energy Conservation Projects in the Pacific Northwest”, Energy Engineering, Vol. 90, No. 4, 1993.

14. N. C. Bleckerr, "Benefits of Energy Efficient Lighting”, Energy Engineering, Vol. 90, No.6, 1993.

15. Umesh Saxena, Rammohan Modepalle, "Development of Self Assessment Tool for Energy Conservation”, Energy Engineering, Vol. 91, No. 3, 1994. 
16. Michael Brown, Derek Smid, Bernanrd Matthews, Michael McKeon, Sirkka Numminen, "Increasing the Implementation of Energy - Efficient Technologies Among U. S. Manufacturers”, Energy Engineering, Vol. 91, No. 6, 1994.

17. Francis Wai Hung Yik, John Burnett, "An Experience of Energy Auditing on a Central Air-conditioning Plant in Hong Kong”, Energy Engineering, Vol. 92, No. 2, 1995.

18. Umesh Saxena, Kurt Bohlman, "A Self Assessment Tool for Energy Management for the Cardboard Industry”, Energy Engineering, Vol. 92, No. 3, 1995.

19. Harry Taplin, "Reducing Energy Costs of Boilers", Energy Engineering, Vol.92, No. 5, 1995.

20. Robert Hoshide, “Effective Energy Audits”, Energy Engineering, Vol. 92, No. 6, 1995.

21. Alex Lee, Jay Zarnikau, Philip Schmidt, "Energy Savings Opportunities in the Texas Oil and Gas Production (SIC 13) Industry”, Energy Engineering, Vol. 93, No. 3, 1996.

22. B. Gopalakrishnan, R. W. Plummer, S. Nagarajan, "Energex: Expert Systems for Industrial Energy Conservation and Management”, Energy Engineering, Vol. 94, No. 2, 1997.

23. Gurvinder Singh, Doug Presny, Charlie Fafard, "Energy Audits Reveal Significant Energy Savings Potential in India's Commercial Air-conditioned Building Sector”, Energy Engineering, Vol. 94, No. 5, 1997.

24. Martin Mozzo Jr., "Environmental Benefits from Energy Projects”, Energy Engineering, Vol. 95, No. 3, 1998.

25. Scott Dunning, Bruce Segee, Vincent Allen, "A Self - Assessment Software Application for Industrial Manufacturers”, Energy Engineering, Vol. 96, No. 1, 1999.

26. Sriram Somasundaram, Steve Parker, Meredydd Evans, Daryl Brown, "Ukraine: Emerging Market for Industrial Energy Efficiency Opportunities”, Energy Engineering, Vol. 96, No. 2, 1999.

27. William Meffert, “Energy Assessments in Iron Foundries”, Energy Engineering, Vol. 96, No. 4, 1999. 
28. Warren Heffington, Galyn Gafford, "A Case Study of an Industrial Assessment Center Extended Assessment”, Energy Engineering, Vol. 96, No. 4, 1999.

29. B. Gopalakrishnan, R.W. Plummer, N. Alkadi, "Analysis of Energy Conservation Opportunities in Glass Manufacturing Facilities”, Vol. 98, No. 6, 2001.

30. Frederick Fendt, “A Highly Successful Holistic Approach to a Plant-Wide Energy Management System”, Steam Digest, Office of Industrial Technologies, 2001.

31. Joel Weber, "Celanese Chemicals Clear Lake Plant Energy Projects Assessment and Implementation”, Steam Digest, Office of Industrial Technologies, 2001.

32. David Jaber, "Business Benefits from Plant Energy Assessments and Energy Management”, Steam Digest, Office of Industrial Technologies, 2001.

33. Onsite Energy Corporation, “Chemical Industry: On-site Power Market Assessment”, Carlsbad, California, September 1997.

34. OIT Clearinghouse, "Vision: Results for Today. Leadership for Tomorrow", Office of Industrial Technologies, February 2001.

35. Chemical Industry Analysis Brief, http://www.eia.doe.gov/emeu/mecs/iab/chemic als/index.html

36. Office of Technology Assessment, "Industrial Energy Use”, U. S. Congress, Washington, D. C., June 1983.

37. Gary Shafer, "Efficient Compressed Air Management: A Systems Approach", Plant Engineering, February 1997.

38. EIA Historical Environmental Data, http://www.eia.doe.gov/neic/historic/henviro nment.htm

39. Jenkins Gwilym, Youle Philip, "Systems Engineering”, C. A. Watts \& Co. Ltd., London, 1971.

40. Morton J.A., "Integration of Systems Engineering with Component Development”, Electrical Manufacturing, Vol. 64, pp. 85-92, August 1959.

41. Office of Industrial Technologies, “DOE’s Industrial Assessment Centers”, U. S. Department of Energy, October 1995.

42. North American Insulation Manufacturers Association, “3E Plus”, http://www.pi peinsulation.org/pages/home.html, Alexandria, VA, Version 3.1.

43. Energy Efficiency Handbook, January 1998, U.S. Department of Energy. 
44. OIT: Plant-Wide Assessments, April 2003, http://www.oit.doe.gov/bestpractices/ plant_wide_assessments.shtml.

45. Industrial Technologies Program, "Plant-Wide Assessments Help Industry Identify Energy and Cost Savings Opportunities”, Washington, DC, October 2002.

46. FlowStream ${ }^{\circledR}$ OFS Series, Mass Flow Meters for Gases, Universal Flow Monitors, Inc., Hazel Park, MI, July 2003.

47. Alan T. J. Hayward, “Flow Meters”, The Macmillan Press Ltd., London, 1979.

48. S. G. Dukelow, “Improving Boiler Efficiency”, Cooperative Extension Service, Kansas, March 1981.

49. Conservation Paper No. 46, “Economic Thickness for Industrial Insulation”, U.S. Federal Energy Administration, Washington D.C., August 1976.

50. Hugh J. Miser, Edward S. Quade, "Handbook of Systems Analysis - Craft Issues and Procedural Choices”, North - Holland, New York, 1988. 